\title{
Ready set go? : a study of the development and implementation process of the BeweegKuur
}

Citation for published version (APA):

Helmink, J. H. M. (2012). Ready set go? : a study of the development and implementation process of the BeweegKuur. [Doctoral Thesis, Maastricht University]. BOXPress. https://doi.org/10.26481/dis.20121207jh

Document status and date:

Published: 01/01/2012

DOI:

10.26481/dis.20121207jh

Document Version:

Publisher's PDF, also known as Version of record

\section{Please check the document version of this publication:}

- A submitted manuscript is the version of the article upon submission and before peer-review. There can be important differences between the submitted version and the official published version of record.

People interested in the research are advised to contact the author for the final version of the publication, or visit the DOI to the publisher's website.

- The final author version and the galley proof are versions of the publication after peer review.

- The final published version features the final layout of the paper including the volume, issue and page numbers.

Link to publication

\footnotetext{
General rights rights.

- You may freely distribute the URL identifying the publication in the public portal. please follow below link for the End User Agreement:

www.umlib.nl/taverne-license

Take down policy

If you believe that this document breaches copyright please contact us at:

repository@maastrichtuniversity.nl

providing details and we will investigate your claim.
}

Copyright and moral rights for the publications made accessible in the public portal are retained by the authors and/or other copyright owners and it is a condition of accessing publications that users recognise and abide by the legal requirements associated with these

- Users may download and print one copy of any publication from the public portal for the purpose of private study or research.

- You may not further distribute the material or use it for any profit-making activity or commercial gain

If the publication is distributed under the terms of Article $25 \mathrm{fa}$ of the Dutch Copyright Act, indicated by the "Taverne" license above, 


\section{Ready Set Go?}

A study of the development and implementation process of the BeweegKuur

Judith Helmink 


\section{Colophon \\ Cover design: Debby van Hees \\ Cover illustration: Tom van Wanrooy \\ Production: proefschriftmaken.nl || Uitgeverij BOXPress \\ ISBN:978-90-8891-534-5}

(C) Judith Helena Maria Helmink, 2012

The research presented in this thesis was performed at the department of Health Promotion at Maastricht University, the Netherlands. The work in this thesis was performed within the NUTRIM School for Nutrition, Toxicology and Metabolism, Maastricht University Medical Centre+.

All rights are reserved. No part of this book may be reproduced or transmitted in any form or by anymeans, without the written permission from the author or, were appropriate, the publisher of the article. 


\title{
Ready Set Go?
}

\author{
A study of the development and \\ implementation process of the BeweegKuur
}

\section{Proefschrift}

Ter verkrijging van de graad van doctor

aan de Universiteit Maastricht, op gezag van de Rector Magnificus,

Prof. dr. L.L.G. Soete

volgens het besluit van het College van Decanen

in het openbaar te verdedigen op

7 december 2012 om 10 uur

door

Judith Helena Maria Helmink

Geboren op 21 april 1983

Maurik (Nederland) 
Promotor

Prof. dr. Nanne de Vries

\section{Co-Promotor}

Dr. Stef Kremers

\section{Beoordelingscommissie}

Prof. dr. Matthijs Hesselink, voorzitter

Dr. Patricia van Assema

Prof. dr. Christophe Delecluse

Prof. dr. Maria Koelen

Prof. dr. Trudy van der Weijden 


\section{Contents}

Chapter 1

Introduction

Chapter 2

Development and implementation of a lifestyle intervention to promote physical activity and healthy diet in the Dutch general practice setting: the BeweegKuur programme

Chapter 3

Factors determining the motivation of primary health care professionals to implement and continue the 'Beweegkuur' lifestyle intervention programme

Chapter 4

Sitting time and body mass index in diabetics and pre-diabetics willing to participate in a lifestyle intervention

Chapter 5

Baseline predictors of maintenance of intervention-induced changes in physical activity and sitting time among diabetic and pre-diabetic patients: A descriptive case series

Chapter 6

69

The BeweegKuur programme: A qualitative study of promoting and impeding factors for successful implementation of a primary healthcare lifestyle intervention for overweight and obese people

Chapter 7

Combined lifestyle interventions in the Netherlands: experiences gained with the BeweegKuur intervention

Chapter 8

General Discussion

References

Summary

Samenvatting

Dankwoord

Curriculum Vitae

Publication List 



\section{Chapter 1}

Introduction 
In 2007 the Dutch minister of Health, Welfare and Sports (VWS), Mr Ab Klink, published the government's 'Vision on Health and Prevention 2007-2011'. This policy framework letter indicated that prevention was one of the most important ways to decrease the costs of health care. As a consequence, the Dutch Ministry of VWS commissioned the Netherlands Institute for Sport and Physical Activity (NISB) to develop a minimal intervention strategy to improve people's physical activity behaviour. NISB started the systematic development of a theory- and evidence-based lifestyle intervention for pre-diabetes and diabetes patients in 2007. The name chosen for this strategy was BeweegKuur (Dutch for 'Exercise Therapy').

BeweegKuur is a 1-year lifestyle intervention tailored to the individual needs of patients, focusing on changes in physical activity and dietary behaviour, to support the prevention and treatment of type 2 diabetes mellitus. Participants are referred by their general practitioner (GP) to a lifestyle advisor (LSA), usually the practice nurse or a physiotherapist. Based on specific inclusion criteria and in close consultation with the patient, an individual exercise programme is designed and supervised by the LSA. This programme can be attended at existing local exercise facilities or (temporarily) under the supervision of a specialized exercise coach or physiotherapist. All participants are also referred to a dietician and receive diet-related group education. The BeweegKuur intervention was developed with the aim to become an effective and feasible primary care based intervention, and the ambition was to have the intervention included in the Dutch basic health insurance scheme by 2012. In 2009, the Dutch college of health insurers (CVZ) concluded that combined lifestyle interventions, such as BeweegKuur, are cost-effective and could be reimbursed under the Dutch basic health insurance scheme [1].

\section{Background of the problem}

The numbers of people who are overweight or obese are increasing rapidly in Western societies [2]. In 2009, approximately half of the Dutch adult population (47\%) were overweight or obese [3]. Overweight and obesity result from a positive energy balance, in which the energy input from food consumption is higher than the energy expenditure, which is partly determined by physical activity [4]. Obesity in turn increases the risk of developing type 2 diabetes, cardiovascular diseases, some types of cancer and psychosocial problems $[5,6]$. In the Netherlands, 740,000 people were diagnosed with diabetes in 2007, 90\% of those had type 2 diabetes [7]. The number of people with diabetes in the Netherlands is expected to have doubled by 2025, partly due to the ageing of the population and the growing numbers of people with obesity [4]. The quality of life of people with diabetes is lower than that of people without this chronic disease, especially in terms of well-being and physical functioning [8]. This quality of life is reduced because at least $40 \%$ of the people with type 2 diabetes suffer from chronic complications, such as cardiovascular diseases, neuropathy, retinopathy and renal failure [9]. These complications limit a person's mobility, and thereby also Their ability to be physically and socially active, which results in a reduced ability to live independently and in a lower quality of life [8]. 
Studies have shown that the development of type 2 diabetes and its complications can be and that pharmaceutical treatment can be postponed, by getting physically inactive people with diabetes to become active $[10,11]$. Physical activity is associated with and/or leads to favourable changes in body composition and a decrease in blood pressure. Physical activity also results in favourable effects on the regulation of blood glucose levels [12]. Finally, exercise and healthy dietary behaviour are not only important in the treatment of type 2 diabetes, but they also reduce the risk of developing diabetes, and are therefore important primary prevention measures [13].

However, it is not only increased physical activity levels and a healthy diet which can prevent overweight and obesity; a decrease in sedentary behaviour is also important. Sedentary behaviour can be defined as activities that do not increase energy expenditure substantially above the resting level, such as sitting, lying down or viewing television [14]. Sedentary behaviour cannot be simply equated with lack of physical activity $[14,15]$; they are two independent behaviours with different effects on health outcomes [16, 17]. Accumulating evidence indicates the importance of reducing sedentary behaviour in addition to increasing physical activity $[18,19]$. The potential negative effects of excessive sedentary behaviour will not be compensated by a few bursts of moderate to vigorous activity per week [20].

\section{Interventions to reduce the incidence of type $\mathbf{2}$ diabetes mellitus}

Several programmes have been developed to reduce the incidence of type 2 diabetes in high-risk groups. The Finnish Diabetes Prevention Study [10], the Diabetes Prevention Programme [21] and the SLIM study [12, 22] have successfully focused on lifestyle changes, in particular on changes in diet and physical activity, in relatively controlled research settings. Because of the gap between theory and practice, however, the challenge is to make these interventions suitable for 'real world' settings [23].

Several studies have examined the effect of lifestyle intervention programmes delivered through primary health care (e.g., [24-27]). Primary care is considered to offer good opportunities for implementing lifestyle interventions in many countries. GPs see their patients regularly and are aware of their social environment [28]. In the Netherlands, diabetic patients usually visit their GP or practice nurse for a check-up every three months. The results of the abovementioned studies showed that lifestyle interventions delivered through primary care can be effective, although the effects on lifestyle behaviours they found were small [29]. A Danish study showed that participants had increased their physical activity levels after the intervention in five similar exercise-on-prescription programmes. The study also found that between three and six participants had to enrol in the programme for one participant to considerably increase their physical activity behaviour [30]. A meta-analysis by Williams and colleagues [29] found that to get one individual to start exercising for 30 minutes a day (at moderate intensity) on a minimum of five days a week, 17 inactive adults had to be referred to an intervention [29]. Nevertheless, a review by Garrett and colleagues [31] showed that exercise-on-prescription interventions delivered through primary care are more cost-effective than intensive gym-based or instructor-led interventions. 


\section{Implementation of combined lifestyle interventions in primary care}

Although delivery through the primary care system might be effective, the question remains whether all primary care providers are willing and able to effectively implement preventive lifestyle interventions. Several factors have been shown to influence the successful implementation of lifestyle programmes in primary care. The first elements that are needed are an active patient role, a GP who takes enough time to discuss the safety and benefits of an intervention [32], and assistance from the programme designers for GPs during the implementation of the programme [32]. A barrier to the implementation of lifestyle programmes delivered through primary care is GPs' subjective lack of time [33]. Although effective training and materials could help overcome this barrier [33], it is quite possible that a GP may not be the most appropriate person to act as a lifestyle advisor. Some interventions have therefore transferred the central role in the intervention from the GP to other primary care providers, such as specifically trained experts or practice nurses [34, 35]. A review on task delegation from GPs to practice nurses in primary care for people with diabetes showed promising results in terms of improving patient care [36]. In addition, it may be more cost-effective to have an intervention delivered by a nurse than by a GP [31].

\section{Barriers affecting intervention participation}

It is not only the health care providers who may encounter barriers in their efforts to implement an intervention; the same may be true for participants of the intervention. Such barriers may include embarrassment about physical appearance, religion and culture, lack of time, inability or unwillingness to arrange a babysitter and unwillingness to attend during evening hours [37]. Participants have also frequently reported a lack of social support or low self-efficacy (i.e. they think that they are not able to exercise) [37]. Apart from personal factors, certain external factors may also discourage potential participants from exercising, for example high costs, distance to exercise facilities [37], an intimidating exercise environment, inadequate supervision and unfavourable opening hours [29]. For some persons, being referred by their GP is an important impetus for participation, because it provides a legitimate reason for starting to exercise [37]. To overcome potential barriers and to identify success factors for an intervention, it is important for its developers to monitor the implementation by means of dissemination research.

\section{Implementation research}

Why is it important to monitor a new intervention with implementation research? Although programme designers may have carefully designed the intervention and based it on a sound theoretical foundation, implementation in 'real life' may still reveal a gap between theory and practice [38]. The intervention may not be entirely suitable for its intended users. Implementation research can reveal factors that promote or hinder the process of implementation of the intervention, so that it can be improved so as to be better suited to 'real life'. 
The process of disseminating an intervention involves three phases for the user. The first phase involves adoption, which implies that an individual or organization becomes willing to participate in an intervention [39]. The second phase is implementation, the process in which an intervention actually gets used within a setting [39]. In this phase, users of the intervention may partially reinvent the intervention in such a way that its suits the organization better. The final phase is sustainability, in which the intervention has been implemented and the user decides whether to continue using it [39]. A classification proposed by Rogers [39] is often used to define adopter categories at individual level, that vary based on differences in the speed of adoption. The first category is that of the 'innovators', individuals who tend to adopt an innovation immediately after its release. This group is followed by the 'early adopters', who tend to be opinion leaders in most systems. They are respected and are important for the adoption of the innovation by the other adopter categories [39]. The 'early majority' is a group with many social and informal contacts, and they tend to adopt an innovation after a variable period of time. Individuals in the 'late majority' category are sceptical about the innovation. The final group is that of the 'laggards', who are characterized by a considerable resistance to the innovation [39].

Successfully implementing an innovative intervention requires an analysis of the promoting and impeding factors in relation to the implementation. This analysis can be based on the diffusion of innovation framework developed by Paulussen and colleagues [40, 41]. This framework takes the characteristics of the user, the organization, the innovation and the political context into account.

The characteristics of the user can be evaluated using the Theory of Planned Behaviour [42] and the Social Cognitive Theory [43] as theoretical frameworks, since the behaviour of a healthcare providers can be viewed as an intentional action which is controlled by the person's beliefs $[40,44]$. In the Theory of Planned Behaviour (TPB), the intention to perform a particular behaviour is the most proximal determinant of the actual behaviour, and is in turn best predicted by attitude, subjective norm and perceived behavioural control [42]. The characteristics of the user are the most direct predictors of the adoption, implementation and continuation of an innovation [41, 45]. The Social Cognitive Theory states that learning occurs in a social context. Two key concepts of this theory are the outcome expectancies (the expectations about outcomes of the behaviour) and self-efficacy (one's confidence in the ability to perform certain behaviour). The Social Cognitive Theory also emphasizes reciprocal determinism in the interaction between a person and this environment [43].

The second type of factor that can influence the implementation process is the characteristics of the socio-political context, such as social support, rules and legislation. This group of characteristics includes not only laws and regulations, but also funding (for example subsidies) [46].

The characteristics of the innovation itself are also important. Five factors can be distinguished [39]. The first factor is relative advantage: the user of the innovation should be able to see the advantages of the innovation in comparison with the old situation. The second factor is compatibility, that is, whether the innovation is compatible with the adopter's values, past experience and perceived need. In our case, this means whether the intervention fits the primary health care facility where it is to 
be implemented. The next factor is complexity. If an intervention is too complex, potential users will not be willing to adopt it. The fourth factor is trialability, which means the degree to which an individual user can experiment with the intervention. Finally, observability is the extent to which the results of the innovation are visible to others [39]. The final factor in diffusion concerns the characteristics of the organization, for example its structure, as well as the collaboration between different parties [39].

Implementation studies can make use of various methods, including qualitative research methods (such as observations, interviews or focus groups) or quantitative methods (involving questionnaires or anthropometric assessments) [47]. Furthermore, implementation research can focus on patients as well as health care providers. The studies reported on in the present thesis involved various types of research (questionnaires, interviews and focus groups) and also focused on various health care providers (GPs, physiotherapists, practice nurses and dieticians) as well as patients.

\section{Development of the BeweegKuur intervention}

The development process of the BeweegKuur started in 2007, when the Dutch Minister of Health, Welfare and Sports commissioned NISB to develop an intervention. Maastricht University became involved in the project by the end of 2007, when it was asked to monitor the intervention by means of implementation research. Figure 1.1 shows the timeline for the iterative development and implementation process of the BeweegKuur intervention.

Chapter two of this thesis presents a detailed description of the systematic development process of BeweegKuur. Briefly, the intervention was implemented in the Netherlands with the help of advisors of the Regional Support Structure for Primary Health Care (ROS). The Netherlands is divided into 20 ROS regions. The primary task of an ROS advisor is to stimulate integration and cooperation within primary care; they also offer assistance in primary care. The ROS plays a key role in the local coordination of the BeweegKuur programme, with the aim of creating a solid local infrastructure for prevention in primary care.

In March 2008, the prototype intervention was implemented in 19 primary health care centres in the Netherlands (in seven ROS regions), and the first patients were enrolled in the intervention. In April, a process evaluation was conducted with all health care providers participating in the intervention. At the end of 2008, a second questionnaire assessed the opinion of health care providers about BeweegKuur.

During 2008, the prototype of the BeweegKuur intervention was adjusted. By the end of 2008, NISB had completed an improved version of BeweegKuur, which was then implemented in the primary health care centres of 14 ROS regions. By the end of 2009, BeweegKuur had been implemented in all (20) ROS regions. 
Figure 1.1: Timeline of the iterative BeweegKuur development and implementation process.

2007

September

Assignment 'Minimal Intervention Strategy Exercise' to NISB

- Version 1: 'Minimal Intervention Strategy

October Exercise'

November

- Expert meeting

- Focus groups with patients

December

Draft version prototype

Framework letter of Minister Klink: Vision on Health and Prevention 2007-2011

- Project plan BeweegKuur ready

- Minister decides to finance project

- Maastricht University involved in the project

\begin{tabular}{ll|} 
January & $\begin{array}{l}\text { Implementation of the draft prototype in } \\
19 \text { primary health care centers (7 ROS } \\
\text { regions) }\end{array}$ \\
April & $\begin{array}{l}\text { Chapter } 3 \text { is based on these data } \\
\downarrow\end{array}$ \\
October & $\begin{array}{l}\text { Second measurement healthcare } \\
\text { professionals }\end{array}$
\end{tabular}

2008

December

Review by project partners

Dissemination draft prototype

First patients starting with the BeweegKuur

$\boldsymbol{4}$

Chapter 4 is based on these data

Development of the improved prototype

2009

January Further development BeweegKuur for

Implementation improved prototype in 14

ROS regions

- Review by project partners

May obese and overweight people

ilot implementation of the BeweegKuur

in 5 healthcare centers.

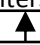

r.

2010

Chapter 6 is based on these data

January

April Improved prototype BeweegKuur ready to implement

June

Chapter 5 is based on these data

2 year follow-up measurement (pre)

October diabetes patients (started in 2008)

Implementation of the BeweegKuur

prototype in 20 ROS regions

- Start cost-effectiveness studies BeweegKuur

New Governement in the Netherlands

Political discussions on the BeweegKuur in health insurance package

une Chapter 7 reflects on the development and implementation process of the in

BeweegKuur 2007-2012 2011

BeweegKuur will not be part of the basic

health insurance 2012 
NISB continued by developing a BeweegKuur intervention for obese and overweight people in 2009. In October 2009, the prototype of this version of Beweegkuur was implemented in five pilot regions, where it was evaluated by means of questionnaires, interviews and focus groups. After six months, the BeweegKuur for obese or overweight people was further adjusted to meet the specific needs of patients and health care providers, and was then implemented in all primary health care centres participating in the BeweegKuur programme. By the end of 2010, a new Dutch government was installed, which decided in 2011 that the BeweegKuur intervention was not going to be covered by the basic health insurance scheme in the Netherlands.

\section{General outline of the thesis}

This thesis presents studies into the development and implementation of the BeweegKuur intervention. Chapter 2 discusses its development process and content, using a model of systematic health promotion planning.

Chapter $\mathbf{3}$ describes the factors that explain the motivation among health care providers to implement and continue using the BeweegKuur intervention. Two questionnaires were sent to Dutch general practitioners, practice nurses and physiotherapists participating in a pilot study. At the time of the baseline measurement, the health care providers were generally preparing to start the implementation, while the second measurement showed them to be in the process of implementing the programme.

Chapter 4 describes the relationship between sitting time and body mass index (BMI) in patients with diabetes or pre-diabetes who were willing to participate in BeweegKuur. It also identifies demographic and psychosocial associates of the motivation to become more physically active.

The study reported on in Chapter $\mathbf{5}$ used the baseline measurement and a measurement two years after the start of BeweegKuur to establish baseline predictors of the maintenance of intervention-induced changes in physical activity and sedentary behaviour among diabetic and pre-diabetic patients

Chapter 6 of this thesis reports on a study which determined perceived promoting and impeding factors for the implementation of the BeweegKuur programme for overweight and obese people. The study consisted of focus group meetings with intervention participants, interviews with health care providers and a focus group session with dieticians.

Chapter $\mathbf{7}$ describes the evolution of combined lifestyle interventions and the changes in primary care caused by these interventions. The thesis concludes with a general discussion in Chapter 8. 


\section{Chapter 2}

Development and implementation of a lifestyle intervention to promote physical activity and healthy diet in the Dutch general practice setting: the BeweegKuur programme

Published as:

Judith H.M Helmink, Jessie J.M. Meis, Inge de Weerdt, Femke N. Visser, Nanne K. de Vries \& Stef P.J. Kremers (2010) Development and implementation of a lifestyle intervention to promote physical activity and healthy diet in the Dutch general practice setting: the BeweegKuur programme. International Journal of Behavioral Nutrition and Physical activity, 7, 49. 


\begin{abstract}
Introduction: The number of patients with diabetes is increasing. BeweegKuur (Dutch for 'Exercise Therapy') is a Dutch lifestyle intervention which aims to effectively and feasibly promote physical activity and better dietary behaviour in primary health care to prevent diabetes.

Methods: The goal of this paper is to present the development process and the contents of the intervention, using a model of systematic health promotion planning. The intervention consists of a 1-year programme for diabetic and prediabetic patients. Patients are referred by their general practitioner to a lifestyle advisor (LSA), usually the practice nurse or a physiotherapist. Based on specific inclusion criteria and in close collaboration with the patient, an individual exercise programme is designed and supervised by the LSA. This programme can be attended at existing local exercise facilities or (temporarily) under the supervision of a specialized exercise coach or physiotherapist. All participants are also referred to a dietician and receive diet-related group education. In the first pilot year (2008), the BeweegKuur programme was implemented in 7 regions in the Netherlands (19 GP practices and health centres), while 14 regions (41 GP practices and health centres) participated during the second year. The aim is to implement BeweegKuur in all regions of the Netherlands by 2012.

Discussion: The BeweegKuur programme was systematically developed in an evidenceand practice-based process. Formative monitoring studies and (controlled) effectiveness studies are needed to examine the diffusion process and the effectiveness and cost-effectiveness of the intervention.
\end{abstract}




\section{Introduction}

Commissioned by the Dutch Ministry of Health, Welfare and Sports (VWS), a lifestyle intervention called 'BeweegKuur' was developed by the Netherlands Institute for Sport and Physical Activity (NISB) [48]. The BeweegKuur is a lifestyle intervention tailored to the individual needs of patients, focusing on a change in physical activity behaviour and dietary behaviour, to support the prevention and treatment of type 2 diabetes mellitus. The intervention is developed with the aim to become an effective and feasible primary health care based intervention, which in time can be reimbursed under the Dutch basic health insurance scheme. The current paper describes the rationale for the development of the BeweegKuur programme, as well as its development and contents. It also outlines the design of a formative evaluation study of the pilot implementation. Effective interventions should be based on the model of systematic intervention planning and development (Figure 2.1, see also [49, 50]).

Figure 2.1: Model of systematic intervention planning and development

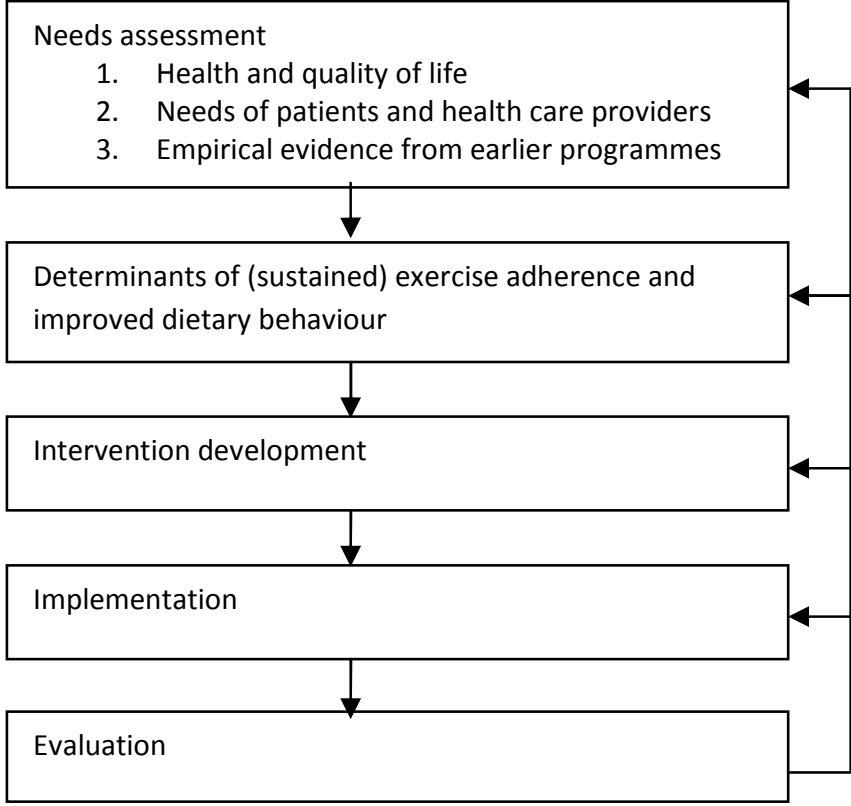

The first step of this planning model comprises a needs assessment. This step involves gathering both quantitative (e.g., literature review) and qualitative (e.g., in-depth interviews) information regarding the needs for intervention development. The needs assessment starts by reviewing health and quality of life. It also reviews personal (i.e. patient-related) and environmental (e.g., health care professionals) factors involved in unhealthy lifestyle, as well as empirical evidence on existing primary health care interventions. The second step of the model explores the determinants of (sustained) exercise adherence and improved dietary behaviour. This is followed by the 
development of a prototype for the intervention (step 3) and its pilot implementation (step 4). All these steps should be evaluated in the fifth step. With respect to the prototype development and pilot implementation, the evaluation is typically and preferably of a formative nature [51]. The structure of this paper follows the steps of this planning model.

\section{Step 1: Needs Assessment}

\section{Health and quality of life}

Approximately 740,000 people in the Netherlands were diagnosed with diabetes in $2007,90 \%$ of whom had type 2 diabetes [7], while 250,000 to 740,000 people are estimated to be unaware that they have the disease [7]. Additionally, approximately 900,000 people aged 60 years and older suffer from early-stage diabetes (Impaired Glucose Tolerance: pre-diabetes) [4]. The number of Dutch citizens with diabetes is expected to have doubled by 2025 , partly due to the ageing population and the increasing number of overweight people [4]. At least $40 \%$ of people with type 2 diabetes suffer from chronic complications, such as cardiovascular diseases, neuropathy, retinopathy and renal failure [9]. These types of complications particularly limit their mobility and, therefore, their ability to be physically and socially active, resulting in a reduced ability to live independently and in a lower quality of life [8]. Physical inactivity, unhealthy dietary behaviour and obesity play a significant role in the development of type 2 diabetes [52]. Currently, at least five million people in the Netherlands are overweight and physically inactive [4]. It has been argued that the greatest benefit to health can be achieved by getting physically inactive people with diabetes to become active, which can delay the development of complications in the long term and support and postpone pharmaceutical treatment $[10,11]$. The advantageous effects of exercise include changes in body composition and a decrease in blood pressure. Exercise also results in favourable effects on the regulation of the blood glucose level [12]. Finally, exercise as well as healthy dietary behaviour are not only important in the treatment of type 2 diabetes, but also decrease the risk of developing diabetes, and are therefore an important primary prevention measure.

\section{Needs of patients and primary health care providers}

Before developing a prototype of the BeweegKuur intervention, a preliminary needs assessment was carried out (see Figure 2.2). A literature search was used to explore existing lifestyle interventions, national as well as international [53]. Based on this literature review, in-depth interviews were conducted with leaders of Dutch national projects relevant to the BeweegKuur programme. The outcomes of these interviews were used to develop the first outline of the intervention. During its development, the outline was discussed with primary health care providers as well as patients by means of face-to-face interviews and focus group sessions. The goal of this discussion process was to see how the draft prototype should be adjusted to fit the needs of both patients and health care professionals. The first step in the process consisted of interviews with project partners and specialists to assess whether the prototype was feasible and to discuss improvements to the prototype. In addition, four expert panels 
were formed in different regions in the Netherlands, each including eight to fourteen people, from different disciplinary backgrounds and with or without experience with lifestyle interventions. The outcomes of these expert panel meetings were incorporated in an improved version of the prototype.

Figure 2.2: Timeline of the BeweegKuur

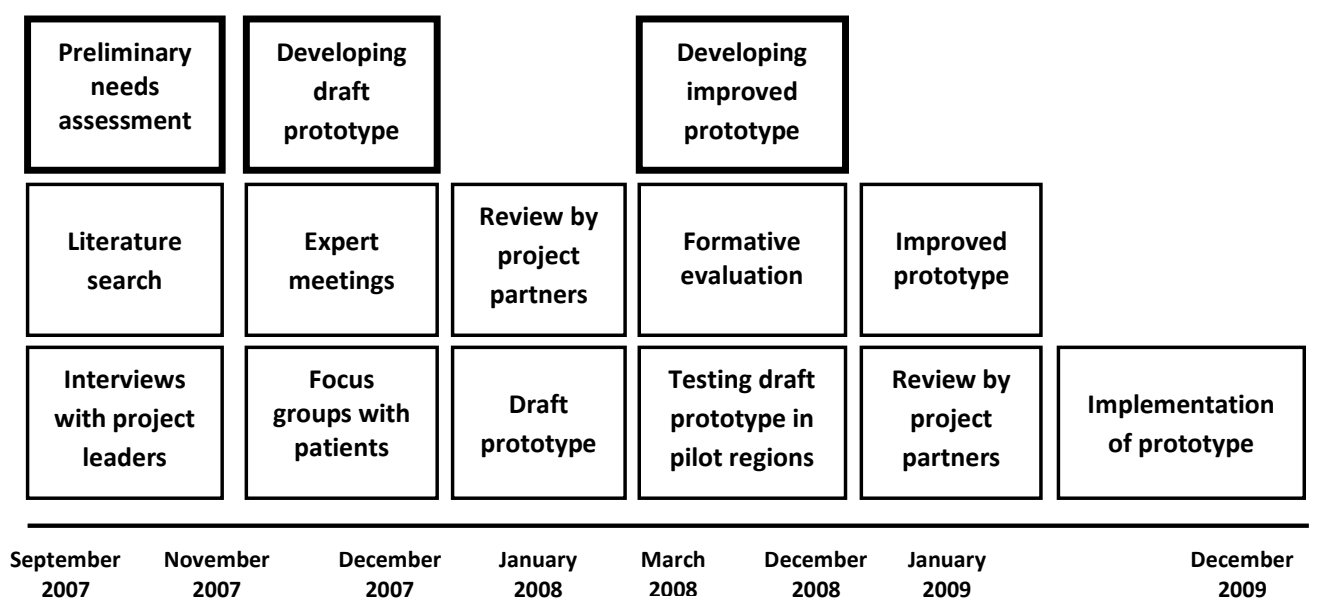

Three focus groups with diabetic and prediabetic patients were then organized to assess the views of diabetic or prediabetic patients regarding the care they would expect, the role of health professionals and the perceived need for the BeweegKuur programme. The main outcomes of the focus group sessions were that referral to BeweegKuur by a general practitioner (GP) would provide additional motivation for patients, which is consistent with previous research [28]. The patients emphasized the importance of close collaboration between the different health care professionals and their need to feel safe with these professionals. Other issues included the importance of a smooth transition to existing local exercise facilities and of low costs. Finally, the patients indicated that they would be stimulated by noticeable results, for example initial loss of weight or an improvement in blood glucose levels.

The results of the focus groups were confirmed in three interviews with patients who were participating in an intervention similar to BeweegKuur. Important aspects they mentioned were close collaboration between health care professionals, personal guidance by a health care professional, a clear structure and exercising in a group with peers. The main reason for attending the lifestyle intervention appeared to be achieving weight loss. The opinions of health care professionals regarding lifestyle interventions were explored in nine face-to-face interviews with GPs, physiotherapists and lifestyle advisors (LSA) who were already participating in a lifestyle intervention. The health care providers affirmed the importance of lifestyle interventions. Important topics they mentioned included the crucial role of motivating patients to start and continue the intervention, and working in a multidisciplinary team. 
Empirical evidence from existing lifestyle interventions

Various relatively controlled lifestyle interventions to prevent type 2 diabetes (i.e. highly supervised and executed within a research context) have been developed and evaluated in the Dutch context (e.g., Study on Lifestyle Intervention and Impaired glucose tolerance Maastricht (SLIM) [12, 22], as well as internationally (e.g., the Diabetes Prevention Program (DPP) [21] and the Finnish Diabetes Prevention Study (DPS) [10]. Although details differ between the various studies in terms of outcomes and purposes, the general purport is that a controlled lifestyle intervention to improve physical activity and dietary behaviour has a positive effect on the health of participants [54]. However, it is not self-evident that relatively controlled lifestyle interventions will also be suitable for wider implementation in practice. The following paragraphs review lifestyle interventions delivered by primary health care centres all over the world and specifically in the Netherlands.

To increase patients' physical activity, interventions have been set up in primary health care settings in various countries, such as the UK [29], Finland [24, 25], the United States [27], New Zealand [33] and Australia [55]. The typical conclusion from these types of intervention studies is that their effectiveness is limited. A metaanalysis showed that 17 inactive adults would have to be referred to an intervention to result in one of them starting to exercise for 30 minutes a day, with at least moderate intensity, on a minimum of five days a week [29]. A potential cause of the limited effectiveness relates to high attrition rates, because the intervention interferes with patients' other activities, is not in line with their capabilities [56] or is not designed and implemented so as to result in sustained behaviour change.

Previous studies have revealed various important implementation issues. An active patient role is required, and GPs should take enough time to convince patients that the intervention is beneficial and safe. 'Exercise-on-prescription' interventions were generally perceived as feasible by participating GPs and patients who voluntarily took part in the intervention. However, little is known about the degree of acceptance and feasibility for patients and GPs who refused to participate in an intervention, and for GPs who were not motivated to implement the intervention. GPs also stated that it is important for them to receive help from the intervention designers in terms of the implementation [32]. This is in line with the results of the implementation of the PACE intervention (Physician-based Assessment and Counselling for Exercise) in the US. More than half of the GPs indicated that, without the training, they would not have been able to introduce the intervention properly [27]. In Australia, research has shown that a prescription for exercise by a GP accompanied by written material led to an increase in self-reported physical activity by inactive individuals within a short period of time. However, this research also showed that a prescription for exercise alone did not result in more physical activity [55]. For example, GPs indicated that they preferred presenting patients with written exercise objectives rather than verbal ones, which also made the GPs feel comfortable about discussing physical activity with patients and prescribing exercise [55]. Although the GPs who participated in the study supported the concept, they indicated that the lack of time presented an immediate and significant obstacle, which might be overcome by suitable training and materials [33]. 
Factors that could discourage participants from exercising include high costs and the distance to exercise facilities; embarrassment about physical appearance; religion and culture; lack of time; inability or unwillingness to arrange a babysitter or attend during evening hours; and lack of support from the social environment [37]. Another barrier to participation in an exercise-on-prescription intervention could be low self-efficacy. External obstacles were also identified, such as an intimidating exercise environment, inadequate supervision during exercise and unfavourable opening hours [29]. Referral by a GP was an important motivation to participate because it forms a strong incentive and provides a legitimate reason for starting to exercise [37].

In the Netherlands, various initiatives have been set up in primary health care settings to increase patients' physical activity behaviour, with names like Big!Move [57, 58], Exercise on Prescription [26, 37] and 'From Complaint to Strength' [59]. The results of these projects were generally favourable in terms of self-reported effect indicators. Participants indicated that they had increased their level of physical activity and that they felt better and healthier [37]. During the exercise programme, the participants developed a more positive attitude towards exercising, and they reported that the social atmosphere and social contacts were important aspects of the intervention [37]. A change was also observed in the number of consultations with their GP, which decreased by $20 \%$ during the Big!Move project $[57,58]$. The projects show that a lifestyle intervention can be effective, when specific barriers are overcome. The BeweegKuur programme tries to overcome potential motivational barriers for the patients by training health care providers to apply motivational interviewing. Health care providers are supported in the implementation of the BeweegKuur intervention by the NISB, which trains all health care professionals involved to work with the intervention. The Regional Support Structure for Primary Health Care (ROS) advisor can assist them in optimizing their implementation of the BeweegKuur programme. The referral by the GP is assumed to provide an important motivation for patients to start exercising, and the adoption of a multidisciplinary approach is expected to increase the success in terms of promoting a healthy lifestyle. Inclusion of the BeweegKuur programme in the Dutch basic health insurance scheme would reduce the financial barrier to patients. Both the evidence base described above and the practice-based information gathered in step 1 of the planning process were incorporated in the draft of the prototype. For more detailed information regarding the content of the intervention, we refer to Step 3 of the planning process.

\section{Step 2: Determinants of (sustained) exercise adherence and improved dietary behavior}

Although many diabetics and prediabetics are able to improve their physical fitness [10], most of them fail to become more physically active and to improve their diet. Developing interventions to achieve this requires thorough insights into the main determinants of (sustained) exercise adherence and improved dietary behaviour.

These determinants of (sustained) exercise adherence and improved dietary behaviour can be classified into several domains, as demographic and psychological influences coexist with social, environmental and wider policy/ legislative 
determinants [60]. All of these factors can, directly or indirectly, influence people's motivation to be physically active and to consume a healthy diet.

Psychological determinants of exercise and dietary behaviour have been extensively studied, often based on socio-cognitive theories such as the Theory of Planned Behaviour. Concepts such as enjoyment of exercise and self-efficacy have been repeatedly found to be positively associated with physical activity [61]. Factors related to healthy food consumption include health consciousness and knowledge of the prescribed number of servings, as well as knowledge of diet-disease relationships $[62,63]$. Specific barriers to exercise in patients with diabetes include perceived difficulty of engaging in exercise and feelings of tiredness [64]. The central construct in this theoretical framework is the intention or level of motivation to change physical activity and/or diet.

Rather than on the level of motivation, Self-Determination Theory [65] focuses on the type of motivation. The theory assumes that motivated behaviour is based on trying to fulfil the three basic psychological needs of competence, autonomy and relatedness to others, and socio-environmental influences that support these three basic needs are expected to promote intrinsic motivation [65]. According to Deci and Ryan [65], intrinsic motivation is linked to greater productivity, cognitive flexibility, and perseverance. Generally speaking, people should have a sense of choice and feel confident about being able to meet their health-related goals. They also need to feel that they are fully responsible for initiating and maintaining healthy behaviour. Previous research showed that when patients perceive their doctor as autonomysupportive, they report greater intrinsic self-motivation for treatment adherence [66]. In order to achieve sustained behavioural change, lifestyle interventions thus need to ensure engagement in physical activities that are intrinsically enjoyed, that participants feel competent at, and that contribute to their sense of autonomy [67]. Furthermore, lifestyle interventions need to enhance autonomous motivation for diet improvement, to facilitate better maintenance of healthy behaviour change $[68,69]$.

People's motivations, abilities and opportunities to change their healthrelated behaviour may also be strongly dependent on the environments they live in. This implies that optimal intervention structures are the result of an investment in enhanced facilitative environments (e.g., a solid local infrastructure in which people can be referred to various local exercise facilities), which may be regarded as a crucial feature to enable sustained behavioural change.

Theory- and evidence-based knowledge about the main determinants of sustained changes in dietary behavior and physical activity has led to the following major change objectives [49] of our intervention. The BeweegKuur programme should:

- promote participants' autonomous motivation for behaviour change,

- inform participants about lifestyle-disease relationships and ways in which they can improve their own health status,

- increase participants' willingness to change their health-related behaviours and support their own initiatives,

discuss difficulties in behaviour change and assist participants improve their problem solving skills, and 
- promote a facilitative environment to engage in sustained physical activity and healthy dietary behaviour.

\section{Step 3: Intervention development}

An elaborate scrutiny of the first two steps resulted in the development of a draft prototype of the BeweegKuur intervention. During the first year of the study (2008), the prototype was continuously being changed, guided by formative research to achieve further improvement [53]. At the same time, a professional development and support manual was developed, based on the experiences of experts and "experts by experience". This took place under the supervision of the Development and Professional Advancement work group, consisting of representatives from the NISB, and all relevant Dutch professional organizations in the field of health care, including NHG (Dutch College of General Practitioners), KNGF (Royal Dutch Society for Physical Therapy), LVG (Association of Organized Primary Healthcare), VSG (Dutch Association for Sports Medicine), NVDA (Dutch Association of Doctors' Assistants), TNO (Netherlands Organisation for Applied Scientific Research), NPi (Dutch Institute of Allied Health Care), LHV (National Association of General Practitioners), DNO (Diabetes and Nutrition Organisation), NDF (Netherlands Diabetes Federation) and DVN (Dutch Diabetes Association).

\section{Outline of the BeweegKuur programme}

The BeweegKuur intervention [48] starts and ends at the GP's practice. The practice staff is responsible for including the patient, coaching and supervising them and referring them to allied health professionals and/or local exercise coaches or a sports physician. The aim of the 12-month intervention is to facilitate transfer to existing local exercise facilities [48].

The BeweegKuur programme is open to prediabetic patients and patients with type 2 diabetes (see Table 2.1 for the inclusion criteria per subgroup). In addition to these criteria, eligible patients must have an inactive lifestyle (i.e. not adhering to the Dutch guideline recommendation of exercising at least half an hour for five or more days a week) and motivation for behavioural change. Exclusion criteria for the BeweegKuur are type 2 diabetes with three or more complications, type 2 diabetes with serious polypharmacy and type 2 diabetes with type 3 hypertension.

Table 2.1: The inclusion criteria per subgroup

\begin{tabular}{ll}
\hline Subgroup & Patient profile \\
\hline Subgroup A & $\begin{array}{l}\text { Impaired fasting glucose (fasting glucose value (finger prick) } \geq 5.6 \mathrm{mmol} / \mathrm{I}- \\
\leq 6.0, \text { or fasting plasma glucose value } \geq 6.1 ; \mathrm{mmol} / \mathrm{I}<6.9)\end{array}$ \\
Subgroup B & $\begin{array}{l}\text { Type II Diabetes (according to } 2006 \text { definition; HbA1c } \geq 7.0 \text { ) and/or RR }> \\
140 / 90 \mathrm{mmHg} \text { and/or side-effects from medication }\end{array}$ \\
Subgroup C & Type II Diabetes well-controlled \\
\hline
\end{tabular}


The goal of the BeweegKuur programme is to achieve health benefits through increased physical activity and improved dietary behaviour for patients with type 2 diabetes or prediabetes. The primary objective is to promote a healthy lifestyle in terms of physical activity and dietary behaviour. Secondary objectives include the improvement of physical parameters, such as $\mathrm{HbA1C}$, hypertension and BMI and the cardiovascular risk profile. Long-term objectives are the prevention of type 2 diabetes in prediabetic patients and lowering the incidence of complications in type 2 diabetes patients.

Theoretical and practical context of the BeweegKuur intervention

The starting point for the BeweegKuur intervention is the assumption that a permanent facilitative environment, autonomy support and involvement of counsellors play a crucial role in encouraging patients to take up and maintain positive lifestyle changes. The integration of the Motivational Interviewing counselling technique [70] with an environmental approach that facilitates opportunities to engage in lifestyle changes could thus be regarded as providing optimal conditions for long-term maintenance. The primary health care setting can be regarded as a good place to start a lifestyle intervention. In the Netherlands, GPs act as the gatekeepers to health care; they see their patients regularly and know their social environment [28]. Most diabetic patients visit their GP or the practice nurse for a check-up every three months, enabling quick selection of eligible patients for BeweegKuur and detection of relapse after inclusion in the programme. Prediabetic patients do not get these regularly check-ups.

In the BeweegKuur intervention, it is the GP who determines whether an individual is eligible for the programme. Coaching and supervision are provided by a lifestyle advisor (LSA, often the practice nurse), based on principles of Motivational Interviewing [70]. Peer feedback meetings are regularly organized for the health care providers. All medical specialists involved in the BeweegKuur programme are offered training in motivational interviewing, consisting of four eight-hour sessions, with the final session after one year. This training course is essential to improve the expertise of the professionals involved in the programme. The Motivational Interviewing course incorporates concepts from Self-Determination Theory [65].

Based on specific inclusion criteria and in close collaboration with the patient, the LSA designs an individual exercise programme, which can be attended in the existing local exercise facilities or (temporarily) under the supervision of a specialized exercise coach or physiotherapist. In addition, all participants are referred to a dietician. During the BeweegKuur programme, the GP practice remains the central location, where patients have frequent contact with the LSA about their progress in the programme and about perceived barriers.

Designed as a multi-disciplinary intervention, the BeweegKuur intervention intends to create cohesion between intervention elements and aims to achieve cooperation between partners at both national and local levels. Thus, the BeweegKuur programme contributes to the development of a solid local infrastructure for prevention. The Regional Support Structure for Primary Health Care (ROS) plays a central role in the local coordination. Good contacts within the multidisciplinary team 
are important. Patients should feel that they are supported by a team which is involved and committed, and that all health care providers involved aim for the same goals. It is crucial for patients' confidence in therapy that health care providers have compatible goals, provide unequivocal recommendations, and fully support, respect and know one another. The LSA is the pivot of the BeweegKuur intervention, and is responsible for unequivocal and smooth communication within the multidisciplinary team and the coordination of individual activities within the BeweegKuur programme.

The BeweegKuur programme combines individual counselling with group counselling. Group counseling can promote group cohesion, which can help motivate patients. Cohesion can be seen as a field of forces that act on the participants to stay in the group [71]. These forces depend on participants being attracted to the group and the ability of the group to mediate important goals for the participants. Several studies have shown that cohesion can be a significant predictor of adherence to exercise [7175]. For example, a study by Kwak and colleagues [73] showed that, regardless of participants' cognition about exercise, a close and bonded walking group led to higher adherence. Therefore, the BeweegKuur intervention uses group education as much as possible, for dietary behaviour as well as exercise [54].

\section{Duration of the BeweegKuur programme}

The BeweegKuur programme takes one year. Coaching by the LSA is gradually reduced during this year, being both more frequent and more intense at the start of the programme. The final appointment with the LSA takes place one year after the start of the intervention. It is assumed that patients who are more active and have improved their dietary behaviour over the year have increased their level of intrinsic motivation towards lifestyle changes, and will be able to maintain these changes in the long run as a result of the continuous provision of a facilitative environment (e.g., [67]).

\section{The exercise settings in the BeweegKuur programme}

The LSA determines the intensity level of the exercise programme that best fits the individual patient. The patient can be referred to three distinct settings for physical activity (see figure 2.3 for the pathway of the BeweegKuur). The first is that of exercising in existing local exercise facilities (the independent exercise setting). This could include walking or cycling, in locally organized groups, with or without supervision. Further options within this setting include dancing, going to the gym or swimming. It is important for the continuation of the patients' physical activity that they intrinsically enjoy the physical activity. They also have to feel competent about engaging in the activity and it should contribute to their sense of autonomy [67]. A tailored exercise programme is designed, based on the patient's caloric expenditure. After the intake procedure and explanation of the exercise plan, the LSA and the patient meet six times during the first year (after 2, 4, 8, 16, 20 and 52 weeks) to discuss progress in terms of exercise as well as dietary behavior and the barriers they perceived.

The second setting is a so-called 'start-up exercise setting', which is supervised by a physiotherapist for four weeks. Finally, the third setting is the 'supervised exercise setting', a three-month training programme supervised by a 
physiotherapist, who designs an exercise program. After the weeks of training with the physiotherapist, the LSA and the patient look for an activity that suits the patient, and the patient is referred to local exercise facilities. During the sessions with the physiotherapist, the patient starts exercising in small groups two or three times a week. The training consists of power, endurance and interval training exercises, designed by a team of specialists [76]. The group sessions can also be used for education on dietary and other behaviour, depending on the patients' preferences. The patients in the second and third settings also have six follow-up meetings with the LSA to discuss the perceived barriers and the progress in terms of exercise and dietary behaviour. The meetings with the LSA and the physiotherapist offer an autonomy supportive environment to achieve better treatment adherence [65]. All patients in the BeweegKuur receive a logbook that assists in the self-monitoring process of the behavioural changes, and that guides and promotes the formation of Action Plans [77].

Patients are referred to one of the three settings depending on the presence of comorbidity, exercise related complaints and strength limitations (as tested with the Cardiac Stress Test). If one or more of these criteria are met, the patient starts in the third setting, with the physiotherapist. If none of these criteria apply, the patient starts in the first setting. If the criteria are not applicable, but the patient needs to overcome certain barriers to get started, they start in the 'start-up exercise setting'.

Figure 2.3: The pathway of the BeweegKuur intervention
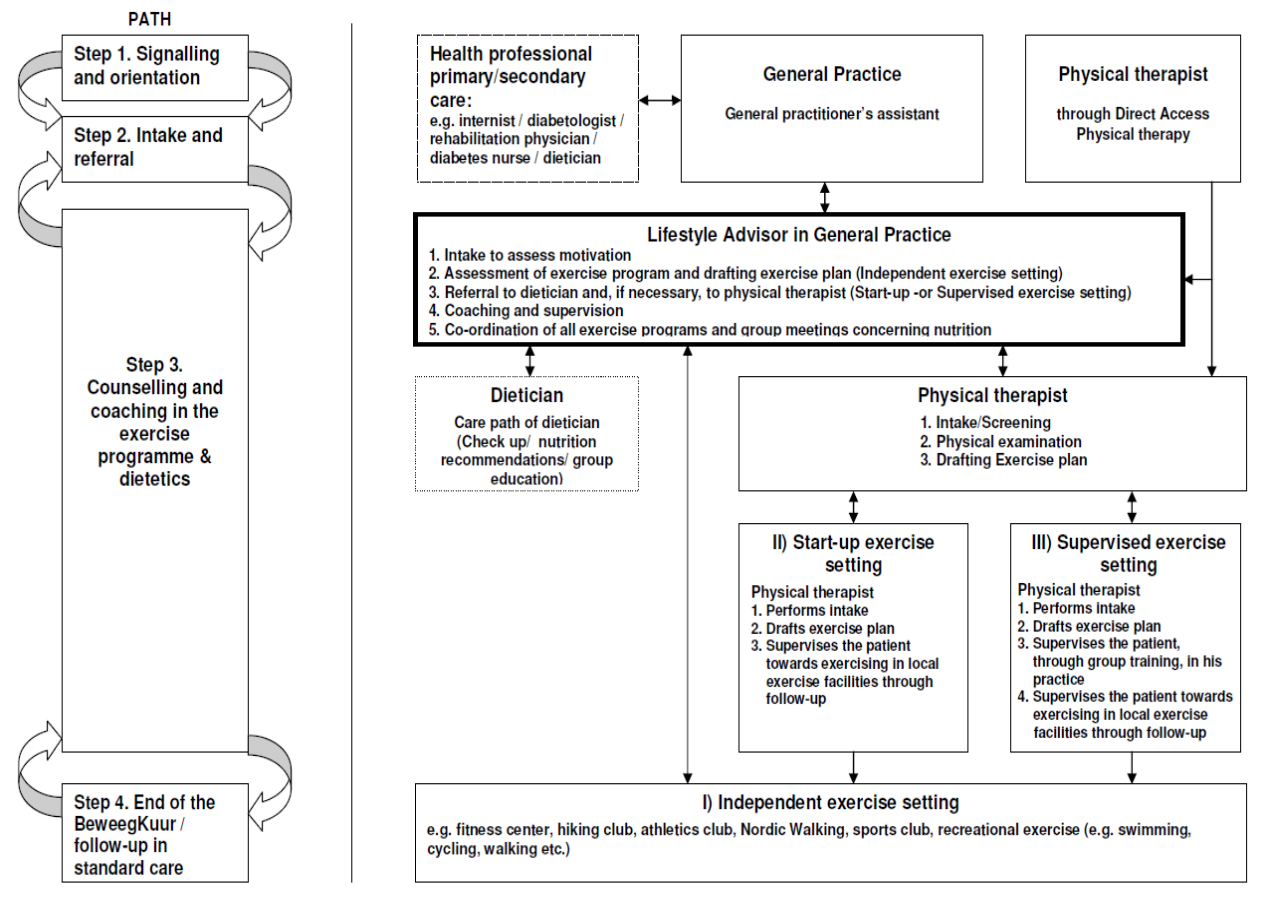


\section{Step 4: Adoption and implementation plan}

In the first year of the BeweegKuur project (2008), seven ROS regions started the intervention. These seven ROS regions implemented the BeweegKuur programme in 19 primary health care centres under relatively strict supervision by NISB. These primary health care centres had a positive attitude towards promoting a healthy lifestyle, and most of them were already experimenting with the implementation of preventive lifestyle interventions. In the second year, 2009, the number of participating ROS regions doubled to 14 , with 41 locations. The selection of the locations was based on the experience of the advisors with the primary health care centres and their belief that a particular practice would be able to implement the intervention at relatively short notice. In 2009, the remaining six ROS regions were preparing to start, so that in 2010 all 20 ROS regions should be participating in the BeweegKuur programme and local exercise networks will be further developed. In 2012, it is expected that all inhabitants of the Netherlands will be able to participate in the BeweegKuur programme in their region if they meet the inclusion criteria. The NISB is developing a plan for further dissemination of the programme.

\section{Step 5: Evaluation and monitoring}

The pilot project for the implementation and dissemination of the BeweegKuur intervention in the first seven ROS regions is being monitored in a formative implementation study [53]. The purpose of this study is to test the BeweegKuur intervention in terms of perceived feasibility, satisfaction and perceived efficacy of the health care providers and the patients who started the BeweegKuur in 2008. The BeweegKuur implementation process is being assessed using interviews with patients, GPs, LSAs and physiotherapists. In addition, patients receive three questionnaires, one at the start of the intervention, one after three months and one after a year. Two questionnaires are sent to the health care professionals (one at the start and one after they have worked with the intervention for 6 months). The evaluation study is based on the systematic approach proposed by Grol and Wensing [46, 78, 79] and the implementation theories proposed by Rogers [39] and Paulussen and colleagues [41, 45]. In addition, after the pilot study, formative monitoring studies and (cost-) effectiveness studies are envisaged to examine the diffusion process and the costeffectiveness of the intervention.

\section{Discussion}

This paper has presented the dynamic development and implementation process of the BeweegKuur intervention. In the first two steps of the systematic intervention planning and development model used in this study, we collected all relevant information for the development of the prototype intervention. In-depth interviews, expert meetings, focus groups and reviewing previous completed and ongoing interventions yielded information about the strengths and barriers of this type of lifestyle intervention in primary care. The information showed that a lifestyle 
intervention can be effective, when specific barriers have been overcome. The BeweegKuur intervention tries to overcome these potential motivational barriers for both patients and health care providers. In this respect, the characteristics of the intervention, i.e. its systematic design and implementation, its theoretical underpinnings and its focus on environmental facilitation, make that the BeweegKuur has the potential to reach moderate effect sizes regarding both short-term change as well as longer-term behavioural maintenance.

There are still some aspects of BeweegKuur that need attention, however. An important point in the BeweegKuur intervention is the access to existing local exercise facilities. During the first pilot year (2008), the network of these exercise facilities was incomplete and the health care providers lacked an adequate overview of the available facilities. This is likely to have resulted in relapse by the participants in the pilot year of the BeweegKuur programme. An additional element of the relapse prevention strategy might be if GPs and LSAs address the patients' lifestyle in follow-up appointments after the end of the BeweegKuur intervention. For example, GPs and practice nurses could be advised to monitor the patients during the regular diabetes appointments. This monitoring of the patient is important, in order to quickly identify lapse and relapse.

Implementation planning for an innovation such as BeweegKuur should take the adopter categories of health care providers into account. Innovators are persons who adopt an innovation immediately after its release [39], and they are followed by the early adopters. These early adopters are characterised by a high degree of opinion leadership in most systems, and are respected and important for the adoption of the innovation by the other adopter categories [39]. The early majority is a category with a lot of informal and social contacts, and will adopt a new idea before the average system member. The late majority is sceptical about an innovation, and the final category consists of the laggards, who are characterized by a considerable resistance to the innovation [39]. In addition, a potential gap exists between the early adopters and the early majority [80]. Early adopters have other goals than the early majority, and the gap between these stages should be taken into account when implementing an innovation [80]. In the first year of the BeweegKuur project, most participating health care professionals were likely to be innovators or early adopters, as a result of the selection procedures. In the coming years of the project, the characteristics in terms of adopter categories are expected to change. The dissemination process of the BeweegKuur intervention will come to a point where the characteristics of the late majority and laggards will prevail. If the BeweegKuur programme were to be included in the basic health insurance package in the Netherlands, this may induce the late majority and laggards to join the intervention. The question remains how these adopter categories can best be approached to ensure intervention quality.

It is conceivable that the BeweegKuur intervention will be adapted to other risk groups than diabetics and prediabetics, such as COPD patients and the obese population. In fact, the prototype of the BeweegKuur programme for diabetic patients will be adapted for overweight and obese people, and will be tested in a limited number of pilot locations in 2010. After the prototype has been adjusted, the aim is to implement the intervention in more primary health care centres. 


\section{Chapter 3}

Factors determining the motivation of primary health care professionals to implement and continue the 'Beweegkuur' lifestyle intervention programme

Published as:

Judith H.M. Helmink, Stef P.J. Kremers, Leonieke C. van Boekel, Femke N. van Brussel-Visser \& Nanne K. de Vries (2012) Factors determining the motivation of primary health care professionals to implement and continue the 'Beweegkuur' lifestyle intervention programme, Journal of Evaluation in Clinical Practice, 18, 682-688. 


\section{Abstract}

Aim: To examine factors explaining motivation among health care professionals to implement and continue a multidisciplinary primary care-based lifestyle intervention, called BeweegKuur, to support prevention and treatment of type 2 diabetes mellitus.

Methods: Questionnaire research with two measurements among Dutch general practitioners, practice nurses and physiotherapists participating in a pilot study. At baseline, professionals were generally preparing to start the implementation. At second measurement, all practices were implementing BeweegKuur.

Results: The results reveal a positive motivation among professionals to implement and continue the intervention. The motivation of practice nurses to continue implementation was lower compared to other professionals. Social support by colleagues, compatibility and perceived relative advantage of the intervention for the professionals were associates of the baseline motivation to implement it. High baseline self-efficacy and profession (i.e. not being practice nurse) positively predicted the motivation to continue the intervention at second measurement.

Conclusions: Professionals in our study can be characterised as innovators or early adopters and inclusion of BeweegKuur in the basic health insurance package may persuade other adoption categories to implement the intervention. An intensified focus on skills building (e.g., Motivational Interviewing skills, general lifestyle counselling skills) is expected to contribute to sustained high-quality implementation of the intervention. 


\section{Introduction}

The incidence of type 2 diabetes mellitus is increasing worldwide. In 2000, the worldwide prevalence of type 2 diabetes was 171000000 and this is expected to increase to 366000000 in 2030. Therefore, intensive efforts to prevent this disease have been advocated [10] and several programmes have been developed with the aim of decreasing the incidence of type 2 diabetes in high-risk groups. Leading prevention programmes, such as the Finish Diabetes Prevention Study [10], the Diabetes Prevention Programme [21] and the SLIM study [12, 22], have successfully focused on lifestyle change, in particular on changes in diet and physical activity, in relatively controlled research settings.

The question arises whether these types of intervention would also be appropriate and effective in 'real-world' settings [23, 81]. General practitioners (GPs) can be regarded as a good starting point for primary care lifestyle interventions in many countries. In the Netherlands, for example, GPs see their patients regularly and are aware of their social environment [28]. Diabetic patients visit their GP or a practice nurse for a check-up every 3 months. The question remains, however, whether all primary care professionals are willing and able to effectively implement preventive lifestyle interventions.

Several studies have examined the effect of lifestyle intervention programmes delivered through primary health care facilities (e.g. [25, 29, 32, 55, 82]). These studies revealed that promoting a healthy lifestyle at the GP's practice can be effective, although the effects on lifestyle behaviours they found were rather small [29]. Successful implementation of lifestyle programmes requires an active patient role, and GPs should take enough time to convince their patients of the benefits and safety of an intervention [32]. GPs have reported that it is important for them to receive assistance from the programme designers for the implementation [32]. GPs need to feel comfortable when discussing these topics and prescribing physical activity to patients, for example, and they prefer to hand over written advice for exercise rather than give verbal instructions [33]. Although GPs generally appear to support the concept of addressing lifestyle in primary care, they indicated lack of time as an immediate, significant obstacle, although adequate training and materials could overcome this [33]. GPs may, however, not be the most appropriate primary care professionals to act as lifestyle advisors [81], so some interventions have aimed at transferring the central role in lifestyle intervention implementation from GPs to other primary care professionals, such as specially trained experts or practice nurses [34, 35, 83]. A recent review on the delegation of tasks from the GP to a (practice) nurse in the diabetes primary care showed promising results in the improvement of patient care [36].

At the request of the Dutch Ministry of Health, Welfare and Sports, the Netherlands Institute for Sport and Physical Activity (NISB) has systematically developed a theory- and evidence-based lifestyle intervention called 'BeweegKuur' (Dutch for 'Exercise Therapy') [48, 81]. BeweegKuur is a multidisciplinary primary care based lifestyle intervention tailored to patients' individual needs, focusing on 
increasing physical activity and improving dietary behaviour, to support the prevention and treatment of type 2 diabetes mellitus. BeweegKuur is envisaged to become an effective and feasible intervention, which in due time would be eligible for reimbursement through the Dutch health insurance system.

The dissemination of the intervention can only be successful if enough is known about the factors that may promote or impede the motivation of health care professionals to implement this lifestyle intervention in routine practice. Health care intervention implementation theory $[40,45]$ assumes that four types of factors predict the motivation to implement and continue innovative interventions like BeweegKuur. The first type of factor that can influence the implementation process is the characteristics of the socio-political context, such as social support, rules and legislation. The second important category is the characteristics of the innovation. Crucial theory-based intervention characteristics are the perceived compatibility, trialability, observability, complexity and relative advantage of the intervention [39]. The third type of factor relates to characteristics of the organization, such as the size and nature of the organization implementing the intervention. Finally, the characteristics of the user are also considered to be primary determinants. These characteristics relate to the health care providers' attitude, subjective norm and selfefficacy towards the implementation of the intervention [41, 42]. We undertook a theory-based determinants study of the motivation of primary care professionals to implement and continue the BeweegKuur intervention.

\section{Methods}

\section{The BeweegKuur intervention}

The BeweegKuur intervention [48] starts at a GP's practice. The aim of the 12-month intervention is to guide participants in achieving a sustained healthy lifestyle [48]. The intervention is intended for people with an impaired fasting glucose and people with type 2 diabetes. In addition to these criteria, eligible patients must have an inactive lifestyle (i.e. not meeting the Dutch guideline recommendation of exercising for at least half an hour on 5 or more days a week) and should be motivated to achieve behavioural change. The intervention was first implemented in a pilot study in 2008. For a detailed description of the BeweegKuur intervention, see Helmink et al. [81].

In the BeweegKuur programme, it is the GP who determine whether individuals are eligible for the intervention. Coaching and supervision are provided by a lifestyle advisor, usually the practice nurse, based on principles of motivational interviewing [70]. All medical specialists involved in the programme are offered training in motivational interviewing. The lifestyle advisor designs an individual exercise programme, in close consultation with the patient. Patients can be referred to an independent exercise setting (local exercise facilities), a start-up exercise setting (training with a physiotherapist for 1 month) or a 'supervised exercise' setting (training with a physiotherapist for 3 months). After training with the physiotherapist, all patients transfer to exercise at local facilities. In addition, all participants are referred 
to a dietician. During the BeweegKuur programme, the GP practice remains the central location, where patients have frequent contact with the lifestyle advisor about their progress in the programme and about perceived barriers. These appointments are more frequent and more intensive in the first 3 months of the programme. The final appointment with the lifestyle advisor takes place 1 year after the start of the intervention.

\section{Data collection}

Both qualitative and quantitative data were gathered. The qualitative data were gathered through in-depth interviews with health care professionals. Before the start of the implementation of the BeweegKuur intervention, four physiotherapists, three practice nurses and two GPs, who were already working with a lifestyle programme in the primary health care setting, were interviewed about impediments to implementation and potential benefits of the intervention. The theoretical framework and the specific information gathered in all interviews in the current study determined the topics for the subsequently developed questionnaires.

The study included two measurements. At the baseline measurement $(t=0)$, the pilot practices $(n=18)$ were generally preparing to start the intervention. At the second measurement $(t=1), 6$ months later, all practices were implementing the intervention, with an average of 21.8 patients (SD 20.1) participating per practice.

\section{Participants and design}

The study participants consisted of primary health care providers relevant for the BeweegKuur intervention (GPs, physiotherapists, practice nurses), who took part in a pilot implementation scheme. The participating practices, primary health care centres or GP or physiotherapy practices were selected because they had a positive attitude towards promoting a healthy lifestyle, and most of them were already experimenting with preventive lifestyle interventions. All participating professionals in the BeweegKuur programme received two questionnaires by mail. The first questionnaire was completed by 59 health care providers (15 GPs, 25 physiotherapists and 16 practice nurses), representing a response rate of $59.8 \% \quad(46.9 \%$ GPs, $62.5 \%$ physiotherapists and $64 \%$ practice nurses). Of the respondents who completed the first questionnaire, 35 also completed the second questionnaire, including 7 GPs, 16 physiotherapists and 12 practice nurses. The response rate at follow-up was $60.3 \%$ (46.6\% GPs, $64.0 \%$ physiotherapists and $75.0 \%$ practice nurses, $83.3 \%$ of whom acted as lifestyle advisors). Dropout analyses showed that gender, age, working in a health care centre nor profession predicted response at the second measurement.

Almost two-thirds $(62.7 \%)$ of the respondents who completed the first questionnaire were women. The mean age of the research population was 42.2 years (SD 10.9), and $59.3 \%$ of the respondents were working in a primary health care centre, while $40.7 \%$ worked in a GP or physiotherapy practice. 
Motivation to implement and continue implementation

At baseline, motivation to implement BeweegKuur was measured with the question: 'How motivated are you to implement the BeweegKuur, on a scale from 1 (totally unmotivated) to 10 (totally motivated)?' The motivation to continue the implementation was measured with the question 'How motivated are you to continue the BeweegKuur after the end of the pilot period, on a scale from 1 (totally unmotivated) to 10 (totally motivated)?' At the second measurement, the question about the motivation to continue the implementation of the BeweegKuur was repeated, to enable us to predict follow-up motivation from the perceived baseline characteristics of the socio-political context, the innovation and the user.

\section{Characteristics of the socio-political context}

The social context was assessed with four items, relating to the social support that health care professionals received from their colleagues within the practice, from fellow professionals, from their professional association and from NISB, the programme designer. An example of such a question was 'I feel that I am supported by other colleagues at this practice in working with BeweegKuur.' These items were measured on a 5-point scale ranging from 'I do not agree at all' (1) to 'I fully agree' (5).

The political context was not assessed in the current study, as it was similar for all participating practices. The study took place in 2008 and 2009, at a time when all professionals were aware that the Ministry of Health, Welfare and Sports intended to propose including the BeweegKuur intervention in the Dutch basic national health insurance package, if its feasibility and cost-effectiveness were proven in a series of studies. If this happened, all primary care providers in the Netherlands would be able to offer their clients the option of enrolling in this lifestyle intervention.

\section{Characteristics of the innovation}

The characteristics of the innovation we assessed included perceived relative advantage for patients, relative advantage for health care providers, and complexity, compatibility, trialability and observability of the intervention [39]. All questions were measured on a 5-point scale ranging from 'I do not agree at all' (1) to 'I fully agree' (5). Relative advantage for patients was measured with five items, e.g. 'If I work with BeweegKuur, I expect this to cause patients to adopt a more active lifestyle.' Reliability analysis revealed a Cronbach's alpha of 0.65 for these items. Relative advantage for health care professionals was measured with eight items, for example, 'BeweegKuur leads to improved communication with other health care professionals' (Cronbach's alpha 0.64). Complexity was measured with four questions (Cronbach's alpha 0.67), for instance, 'The instruction manual for BeweegKuur contains too much text.' Compatibility was measured with six items, for example, 'BeweegKuur fits my work routine' (Cronbach's alpha 0.61). Regarding the implementation of the BeweegKuur, the concepts of trialability and observability are considered to be unidimensional. This enabled them to be measured with one item each 'It is important for me to first test 
BeweegKuur in a pilot setting' (trialability) and 'While working with BeweegKuur, it is important to me that the results of the intervention are visible' (observability).

\section{Characteristics of the user}

The socio-cognitive characteristics of the user were measured by assessing attitude, subjective norm and self-efficacy. Attitude was measured with six items (Cronbach's alpha 0.60) on a 5-point scale from 'I do not agree at all' (1) to 'I fully agree' (5). An example of a question was 'It is important for me that I provide patients with education on exercising.' Subjective norm was measured with four items (e.g. 'The support of my colleagues is important to me' Cronbach's alpha 0.63). Self-efficacy was measured with eleven items (e.g. 'I think I am able to correctly implement BeweegKuur' Cronbach's alpha 0.71).

\section{Statistical analyses}

The statistical analyses were conducted using spss 15.0. Mean scale scores and standard deviations were calculated. In addition, we calculated frequencies of total mean scale scores above (positive) and below (negative) 3 to provide an overview of the distribution of the characteristics of social context, innovation and user. Subsequently, three consecutive hierarchical linear regressions were run using the backward deletion procedure (Pout $>0.05$ ). Dependent variable in each of the regressions was the motivation to implement or continue the implementation of the intervention. Independent variables were background characteristics (gender, age, profession, work organization and number of patients enrolled in the programme) as well as each of the concepts from the theoretical framework (characteristics of the organization, innovation, social context and user).

\section{Results}

Motivation for implementation and continuation of the BeweegKuur intervention On the whole, the health care providers were motivated to implement the BeweegKuur intervention (mean 7.84 on a scale ranged 1-10; SD 1.30; observed range 1-10). A total of $3.4 \%$ of the respondents indicated that their motivation was insufficient (below 6). At baseline, most respondents were also positive about their motivation to continue the implementation after the pilot period, with a mean motivation score of 7.79 (SD 1.29; range 1-10).

The follow-up measurement also showed a generally positive motivation to continue the implementation (mean 7.84; SD 1.24; range 5-10). The motivation among the practice nurses dropped during the study, but this decrease was not significant. The motivation of the GPs and physiotherapists increased during the study, but this increase was not significant either. 
Table 3.1: Mean scores for the characteristics of the socio-political context, the innovation and the user $(N=59)$

\begin{tabular}{|c|c|c|}
\hline & Mean & SD \\
\hline \multicolumn{3}{|l|}{ Motivation to....* } \\
\hline \multicolumn{3}{|l|}{ Implement } \\
\hline GPS & 7.67 & 0.82 \\
\hline Physiotherapists & 7.94 & 1.30 \\
\hline Practice nurses & 7.81 & 0.54 \\
\hline \multicolumn{3}{|l|}{ Continue (to) } \\
\hline GPS & 7.45 & 0.83 \\
\hline Physiotherapists & 7.97 & 1.72 \\
\hline Practice nurses & 7.80 & 0.54 \\
\hline \multicolumn{3}{|l|}{ Continue (t1) } \\
\hline GPS & 8.00 & 1.00 \\
\hline Physiotherapists & 8.30 & 1.13 \\
\hline Practice nurses & 7.17 & 1.27 \\
\hline \multicolumn{3}{|l|}{ Characteristics of the social context** } \\
\hline Social support by direct colleagues & 3.99 & 0.92 \\
\hline Social support by fellow professionals & 3.68 & 0.89 \\
\hline Social support by professional association & 2.53 & 0.93 \\
\hline Social support by the NISB & 3.59 & 0.93 \\
\hline \multicolumn{3}{|l|}{ Characteristics of the innovation** } \\
\hline Relative advantage for patients & 3.75 & 0.36 \\
\hline Relative advantage for health care providers & 3.55 & 0.34 \\
\hline Complexity & 2.98 & 0.63 \\
\hline Compatibility & 3.42 & 0.48 \\
\hline Trialability & 4.17 & 0.53 \\
\hline Observability & 4.05 & 0.54 \\
\hline \multicolumn{3}{|l|}{ Characteristics of the user** } \\
\hline Attitude & 3.55 & 0.52 \\
\hline Self-efficacy & 3.40 & 0.34 \\
\hline Subjective norm & 3.58 & 0.47 \\
\hline
\end{tabular}

*Questions were answered on a scale from (1) totally not motivated to (10) totally motivated

** Questions were answered on a 5-point scale from (1) totally disagree to (5) totally agree

Characteristics of the socio-political context

The majority of the respondents (72.9\%) felt supported by the colleagues within their practice (see Table 3.1 for mean scale scores and standard deviations). More than half (64.4\%) felt supported by colleagues in their professional association and $66.2 \%$ by the institute that designed and implemented the intervention (NISB). More than a third of 
the health care professionals (39.0\%) did not feel supported by their professional association.

\section{Characteristics of the innovation}

Overall, $79.6 \%$ of the health care providers perceived relative advantages of the intervention for their patients and $62.7 \%$ perceived relative advantages for themselves (see also Table 3.1). Some respondents (13.5\%) thought that the intervention was complex, while others $(27.2 \%)$ did not share this opinion and $59.3 \%$ adopted a neutral position in this respect. Half of the respondents thought that the intervention was compatible with their current work, while most of the other respondents $(45.8 \%)$ gave a neutral answer. A large majority (93.2\%) of the respondents agreed with the importance of testing the intervention first in a pilot setting, and the majority $(83.1 \%)$ agreed with the importance of the observability of the results of the intervention.

\section{Characteristics of the user}

The majority (61.0\%) of the respondents had a positive attitude towards the intervention, and $70.2 \%$ perceived a high subjective norm to implement it. High selfefficacy was reported by $35.6 \%$ of the health care professionals, while the others (64.5\%) had a neutral opinion about their self-efficacy.

\section{Multivariate analyses of factors influencing the motivation to implement and to continue}

The regression analysis with the motivation to implement as the dependent variable revealed that $41 \%$ of the variance in motivation was explained by the independent variables (Table 3.2). Higher motivation to implement the BeweegKuur intervention was explained by the perception of a larger relative advantage of the intervention. This relative advantage did not relate to the advantages for the patients but to those for the professionals themselves. In addition, motivation to implement was positively associated with the perceived compatibility of the intervention with routine practice. The more social support health care professionals perceived from their colleagues, the more motivated they were to implement the intervention. The opposite was found for the support by the NISB; increased motivation was associated with less (perceived) support.

The model for the motivation at baseline to continue the implementation of the intervention after the pilot period had a total explained variance of $47 \%$. Gender was a statistically significant predictor; women were more motivated than men. Similar to the motivation to implement, increased motivation to continue was associated with more perceived support by colleagues and less perceived support by the NISB, as well as higher perceived relative advantage for the health care professionals and compatibility of the intervention with routine practice. In addition, the perceived observability of the implementation of the intervention was significantly associated with the motivation to continue implementation. 
Table 3.2: Backwards regression analyses, using motivation to implement and continue the Beweegkuur intervention at to and to continue it at $t 1$ as dependent variables

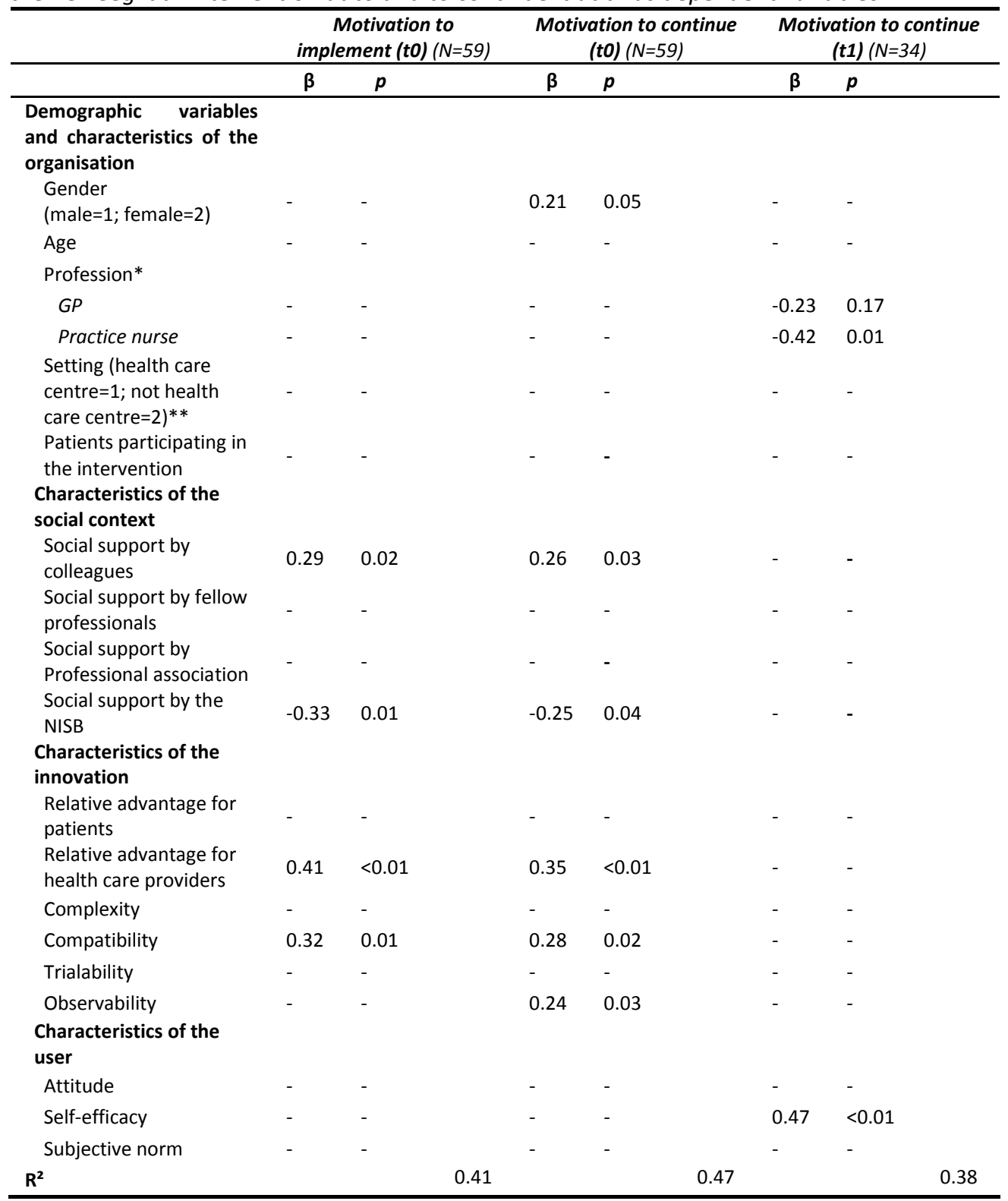

* Reference category= physiotherapists

** 1=health care professional working in a primary health care centre; $2=$ health care professionals not working in a primary health care centre, but in a GP or physiotherapy practice. 
At follow-up, the characteristics of the social context and the innovation lost their predictive value for the motivation to continue implementation. Instead, the profession and the self-efficacy of the respondents became significant predictors. A higher self-efficacy expectation at baseline predicted higher motivation to continue the intervention after the pilot period. Practice nurses were less motivated to continue the intervention than physiotherapists (see Table 3.2).

\section{Discussion}

The primary health care professionals in this study were rather highly motivated to implement the BeweegKuur intervention. This result may be surprising in view of the major shift of focus towards prevention that is represented by the intervention. However, the results need to be interpreted in the context of the natural process of diffusion of innovations as described by Rogers [39]. The high level of motivation can be partly explained by the fact that the health care professionals in this study are best characterized as innovators or early adopters. Innovators are typically persons who adopt an innovation immediately. They are followed by the early adopters and then by the 'early majority', persons with many informal and social contacts, who adopt an intervention before the majority of those involved. The practices that took part in this pilot study were primarily selected on these characteristics, and most had already implemented similar types of lifestyle interventions in their practice in the recent past. Most professionals in our study had a positive attitude towards the implementation of preventive lifestyle interventions in primary care, whereas it is known that there are certain groups of professionals, including some in primary care, that are much more sceptical about this development. In Rogers's theory, such groups are labelled late majority and laggards [39]. Determinants of implementation and continuation in these adopter categories can be expected to differ from those identified in the current study. In a nationwide dissemination process like this, it is generally advocated to focus on the innovators and early majority in the first implementation phase [84]. If the intervention is included in the basic Dutch health insurance package in the future, this may convince the late majority and laggards to implement the intervention in their practices.

Strong points of the present study were the longitudinal design and the theoretical perspective. The BeweegKuur intervention itself is also based on sound evidence [81] and the fact that the intervention has been designated by the Dutch Ministry of Health, Welfare and Sports to become the first preventive intervention to be included in the medical insurance package, which increases the practical relevance of the current study. However, limitations of the study need to be acknowledged. First, the sample size was relatively small and the response rate was approximately $60 \%$, which may have distorted the results. However, dropout analyses showed no selective dropout. Because of the small sample size, we based the results on all health care provides as one group, and the final model might have been different if the groups had been analysed by profession. This was not possible, however, because of the small 
sample size. Second, the dependent variable was measured by only one item, while the independent variables were measured by more items. The third limitation is that the health care professionals may have given socially desirable answers, even though the respondents were guaranteed full anonymity.

The results revealed a lower motivation among the practice nurses to continue the BeweegKuur intervention than among the physiotherapists. Comparable results were found in a study of smoking cessation [85], which found that practice nurses had a lower intention to implement a smoking cessation intervention than the GPs. This lower motivation can be partly explained by the role practice nurses have in lifestyle interventions in general and in the BeweegKuur intervention in particular. Practice nurses typically assume the role of the 'lifestyle advisor', who plays a pivotal role in the intervention, and is responsible for smooth and unequivocal communication within the multidisciplinary team and for the coordination of individual activities within the intervention. A lifestyle advisor therefore spends more additional time counselling participants than the other health care professionals. Thus, the practice nurses probably perceive greater changes in terms of job content and workload as a result of the implementation of the intervention than the other professionals involved. As workloads are generally perceived as high, practice nurses are likely to feel they are not allocated sufficient extra time per included patient to deliver the intervention properly. Although the average motivation to continue was still quite high among practice nurses and did not drop significantly during the pilot period, it is important to monitor this issue. Self-efficacy in particular was found to predict the motivation to continue. Before implementing the intervention, only $35.5 \%$ of the health care professionals reported their self-efficacy to be high. An intensified focus on skills' building (e.g. motivational interviewing skills, general lifestyle counselling skills) is expected to produce higher self-efficacy expectations [49], and may thus ensure sustained high-quality implementation of the intervention.

The perception of social support by colleagues in the practice was important for the professionals to adopt and implement the intervention. Comparable results were found in a study by Harting and colleagues [86] who investigated the determinants of guideline implementation among physiotherapists. These physiotherapists were of the opinion that there should be agreement about work routines within a practice [86]. A motivated multidisciplinary team improves the social support perceived by the health care professionals. GPs have been shown to be more willing to counsel patients in a smoking cessation programme when they perceived support from the practice staff [87]. A literature review by Fleuren and colleagues showed that lack of support and low self-efficacy impede the implementation of innovations [88]. Surprisingly, we found that the motivation to implement and continue the BeweegKuur intervention at baseline was inversely associated with perceived support by the NISB, but it is possible that the support by the NISB was not important to the health care providers at this early stage of implementation. In addition, respondents felt poorly supported by their professional association. Although most of the professional associations were involved in the further development of 
BeweegKuur for the future, their involvement was at national level, which may explain why health care providers did not perceive direct support.

An important associate of the motivation to implement and continue the BeweegKuur intervention was the health care providers' perception of the relative advantage of the intervention for themselves. By contrast, the relative advantage for the patient was not a predictor in this study. Health care providers may first need to see a short-term advantage for themselves, while the longer term advantages for the patients are less salient at the stage of deciding whether to implement a lifestyle intervention programme.

As the implementation continues, the perceived relative advantage for the patients may become more important as a determinant of sustained implementation. Nevertheless, this finding is important for intervention designers and disseminators. It may be especially important to stress the advantages of adopting the intervention for the professionals themselves, including its compatibility with routine practice, rather than to focus on potential longer-term advantages for patients of participating in a lifestyle intervention.

Social context and the characteristics of the innovation appeared to be important in decisions that were made before implementing the intervention, whereas the user characteristic self-efficacy became an important predictor of the motivation to continue implementing after the pilot period. In order to ensure sustained implementation, it is important that health care providers who are working with the programme feel confident about performing their tasks. Although at present, lifestyle counselling is no longer a totally new concept for primary care professionals, the application of specific counselling techniques such as motivational interviewing is relatively new. Motivational interviewing techniques are not easily applied in routine practice and require intensive training and practice support by specific feedback [89]. It is therefore important that health care professionals acquire sufficient skills to continue the BeweegKuur intervention and to facilitate health care providers in this respect. 


\section{Chapter 4}

Sitting time and body mass index in diabetics and pre-diabetics willing to participate in a lifestyle intervention

\section{Published as:}

Judith H.M. Helmink, Stef P.J. Kremers, Femke N. van Brussel-Visser \& Nanne K. de Vries (2011). Sitting time and body mass index in diabetics and prediabetics willing to participate in a lifestyle intervention, International Journal of Environmental Research and Public Health, 8, 3747-3758. 


\section{Abstract}

This cross-sectional study examined the relationship between Body Mass Index (BMI), total sitting time and total physical activity time in a generally overweight or obese population of type 2 diabetics or pre-diabetics willing to participate in a lifestyle intervention [ $n=221,55.1 \%$ male, mean age (SD) 62.0 (9.9), mean BMI (SD) 31.4 (5.0)]. In addition, we aimed to identify demographic and psychosocial associates of the motivation to become more physically active. The measurement instrument was a selfreport questionnaire. Results showed that total sitting time was more closely related to BMI than total physical activity time. Subjects with a higher weight status were more sedentary, but they were also more motivated to be physically active. On the other hand, their self-efficacy to be physically active was lower than subjects with a lower weight status. Lifestyle interventions to decrease the risk of obesity and type 2 diabetes should aim not only at increasing total physical activity time, but also at reducing the total sitting time. Despite generally high levels of motivation among these obese participants, intervention designers and intermediaries should be aware of their low level of self-efficacy towards being physically active. 


\section{Introduction}

Obesity and physical inactivity are the most important risk factors for developing type 2 diabetes mellitus. In the Netherlands, five million people are considered overweight and/or physically inactive [4]. A total of 740,000 people were diagnosed with diabetes in 2007 in the Netherlands, 90\% of them with type 2 diabetes [7]. The incidence of type 2 diabetes is increasing worldwide, and the quality of life of people with diabetes is lower than that of people without this chronic disease, especially as regards wellbeing and physical functioning [8]. Hence, measures to prevent type 2 diabetes have been advocated [10], with lifestyle interventions focusing on the promotion of a healthy diet and physical activity (PA) [11, 12, 24-27, 81]. Lifestyle interventions should not only aim to increase PA, but also to decrease sedentary behaviour, as moderate to vigorous activity a few times a week will not compensate for the potential negative effects of excessive sedentary behaviour [20]. Sedentary behaviour refers to activities that do not increase energy expenditure substantially above the resting level, such as sitting, lying down or viewing TV [14]. It has been suggested that sedentary behaviour should be explicitly measured instead of being defined as a lack of PA [14, 15]. In fact, defining a sedentary population based on low levels or absence of PA might be inaccurate, as sedentary behaviour and PA are two independent behaviours with different effects on health outcomes $[16,17]$. A review by Proper and colleagues showed that there was moderate evidence for an independent relationship between sedentary behaviour and type 2 diabetes [90].

Non-exercise active thermogenesis (NEAT) can play a key role in the energy expenditure of obese people. NEAT is the energy expenditure associated with everyday activity [91]. Altering one's postural allocation from a seated to a standing position or engaging in light ambulation has been shown to significantly increase energy expenditure. A difference in NEAT has been observed between obese and lean subjects [91]. Obese subjects have been shown to sit for 2.5 hours/day more than lean subjects [91, 92]. Underlining the importance of sedentary behaviour in explaining weight status, Santos and colleagues [93] demonstrated that in a general population of Portuguese men, even those meeting public health guidelines for leisure time PA may be at risk for overweight and obesity if they spend a large proportion of their leisure time in sedentary activities. They found that men with a high Total sitting time and with sufficient PA time had a higher average Body Mass Index (BMI) than those with low total sitting time and sufficient PA time. The difference between the groups was statistically significant, though with a small effect size (Cohen's $d<0.1$ ) [93]. In line with the empirical evidence from research about NEAT, we hypothesised that an even larger share of the BMI would be explained by low sitting time in a subpopulation of generally overweight or obese diabetics or pre-diabetics.

The results of the study by Santos et al. [93] also indicated that total sitting time was positively associated with BMI after adjustment for some potential confounders (including total PA time), but they did not correct for potentially relevant cognitive determinants of PA. Theories about behavioural determinants generally 
distinguish three types: outcome expectancies or attitude, subjective norm and selfefficacy. Attitudes are determined by the beliefs about attributes of performing the behaviour [94]. Outcome expectancies are a person's estimate that a given behaviour will lead to certain outcomes [43]. Subjective norm reflects expectations about what people in our social environment want us to do [42]. Self-efficacy (also known as perceived behavioural control) refers to a person's expectation that he or she has the ability to implement the desired behaviour [95].These cognitions have been successfully applied in research on PA [96-100].

The aim of the present study to examine the relationship between BMI, total sitting time and Total PA time in a generally overweight or obese population of diabetics or pre-diabetics, while controlling for demographic and psychosocial associates of the motivation to become more physically active by participating in a lifestyle intervention.

\section{Methods}

\section{Study Design and Sampling}

Participants in this study were people who had agreed to be included in a lifestyle intervention programme called BeweegKuur (see [81] for more information). People with an impaired fasting glucose (pre-diabetics; i.e., fasting glucose range (finger prick) $\mathrm{L} 5.6 \mathrm{mmol} / \mathrm{L}-<6.9 \mathrm{mmol} / \mathrm{L}$ ) and persons with type 2 diabetes (HbA1c L 7.0) were eligible for inclusion in the intervention. Exclusion criteria were type 2 diabetes with three or more complications, type 2 diabetes with serious poly-pharmacy and type 2 diabetes with type 3 hypertension. In this programme, a patient's general practitioner (GP) determines whether the intervention will be offered. After the patient agrees to participate, coaching and supervision is provided by a lifestyle advisor (LSA, often the GP's assistant), based on principles of Motivational Interviewing [70].

The present study used a cross-sectional non-controlled design, based on the (self-reported) baseline data of a longitudinal monitoring study. The LSA was responsible for the distribution of the questionnaire to the patients, who received it at the end of their intake consultation with the LSA. Patients also completed an informed consent form. The questionnaire could be returned to the university free of postage, and participants could win a gift voucher. The questionnaire was completed by 361 persons.

\section{Measures}

Demographic variables assessed in the questionnaire were date of birth, educational level, gender and country of birth. Self-reported weight and height were used to calculate BMI (weight $(\mathrm{kg}) /$ height $\left.(\mathrm{m})^{2}\right)$. PA and sitting time were measured with the short version of the International Physical Activity Questionnaire (IPAQ) [101]. Based on the ACSM/AHA physical activity guidelines, subjects were categorised as: insufficiently active (participants who reported fewer than $150 \mathrm{~min} /$ week of at least moderate-intensity PA or less than $20 \mathrm{~min} /$ week of vigorous-intensity PA) or 
sufficiently active (participants who reported $150 \mathrm{~min} /$ week or more of at least moderate intensity PA or $20 \mathrm{~min} /$ week or more of vigorous-intensity PA) [102]. Total PA time in this article was measured by calculating the amount of moderate and vigorous PA time. The proxy measure of sedentary behaviour was the time spent sitting on an ordinary weekday. This was measured with the question: 'During the last 7 days, how much time did you spend sitting on a week day?' where the respondents could fill out the hours or minutes spent sitting. The sitting time includes time spent at work, at home, while doing course work and during leisure time. Outliers (sitting time on an ordinary day $<120 \mathrm{~min} /$ day) were recoded to the 5 th percentile. Subjects were categorised as having low or high total sitting time, based on the median value for total sitting time found in this sample (360 min/day). Participants were also categorised into the following four groups: low total sitting time/sufficient PA time; low total sitting time/insufficient PA time; high total sitting time/sufficient PA time and high total sitting time/insufficient PA time. Subjects with missing data on any of the PA measures $(n=140 ; 38.8 \%)$ were removed from the further analyses. Drop-out analyses showed that the persons who did not fill in the PA measures did not differ from the complete cases, except for education (more highly educated people being more likely to fill in the PA measures).

The questionnaire items regarding the psychosocial determinants of PA in the BeweegKuur programme were based on the Theory of Planned Behaviour [42], Social Cognitive Theory [103] and the ASE model [95]. Outcome expectancies were measured with eleven items, with answers on a 5-point scale ('I do not agree at all' (1) to 'I fully agree' (5)). Reliability analysis yielded a Cronbach's alpha of 0.79. An example of an item was 'If I participate in BeweegKuur, I will lose weight'. Six items measured the attitude concept $(R=0.65)$. An example of a question was: 'The BeweegKuur programme suits people like me'. Five self-efficacy questions $(R=0.87)$ were asked, again using a 5-point scale with answer categories ranging from 'I do not agree at all' (1) to 'I fully agree' (5). An example of a question was 'I think I will be able to attend the whole BeweegKuur programme.' Three questions were asked regarding subjective norm, measured on a 5-point scale ('I do not agree at all' (1) to 'I fully agree' (5) ( $R=$ 0.87). An example of these questions was 'My family wants me to participate in BeweegKuur'. Motivation to become more physically active at the start of the BeweegKuur intervention was assessed with the question 'How motivated are you to be more physically active', with answering categories on a scale from 0 (not motivated) to 10 (extremely motivated).

\section{Statistical Analyses}

The statistical analyses were conducted using SPSS 17.0. Frequencies were determined to examine the characteristics of the sample and the distributions of age, gender, education, occupation, BMI, sitting time, total PA time, outcome expectancies, attitude, self-efficacy and subjective norm in the sample. Cognitive, motivational and behavioural responses were explored using a Chi-square test and correlations. We correlated demographic characteristics with intermediate (cognitive factors) and 
ultimate outcomes (total sitting time, total PA time, obesity and BMI). Linear regressions were conducted with $\mathrm{BMI}$ as the dependent variable, while independent variables were demographic characteristics (gender, age, education, occupation, country of birth) as well as cognitive factors, total sitting time and total PA time. Logistic regressions were conducted with weight status (obese (1) vs. non-obese (0)) as the dependent variable, and with the same independent variables as the linear regression with BMI. Demographic and psychosocial associates of the motivation to become more physically active were assessed using linear regressions, with motivation as the dependent variable and demographic characteristics, cognitive factors, total sitting time, total PA time and obesity as independent variables. P-values below 0.05 were considered statistical significant, $p$-values below 0.10 were considered being a trend.

\section{Results}

More than half of the respondents were men (55.1\%), with a mean age of 62.0 years. The average BMI was $31.4 \mathrm{~kg} / \mathrm{m} 2$, and $60.6 \%$ of the respondents were obese. The average self-reported PA time was 0.53 hours/day and the average self-reported total sitting time was 6.41 hours/day. The motivation to be physically active was 7.66 (see Table 4.1).

Table 4.1: Descriptive characteristics of the participants $(n=221)$

\begin{tabular}{|c|c|c|c|c|}
\hline & $\%$ & Mean士SD & & $\%$ \\
\hline Gender & & & Level of education & \\
\hline Male & 55.1 & & Low education & 47.3 \\
\hline Female & 44.9 & & Medium-level education & 30.5 \\
\hline Age & & $62.0 \pm 9.9$ & High education & 22.3 \\
\hline Body Mass Index $\left(\mathrm{kg} / \mathrm{m}^{2}\right)$ & & $31.4 \pm 5.0$ & Occupation & \\
\hline $\mathrm{BMI}>30 \mathrm{~kg} / \mathrm{m} 2$ & 60.6 & & Employed & 34.2 \\
\hline Total PA time (hours/day) & & $0.53 \pm 0.87$ & Homemaker & 21.0 \\
\hline Total sitting time (hours/day) & & $6.41 \pm 2.93$ & Retired & 33.3 \\
\hline Outcome expectancies (1-5) & & $3.91 \pm 0.40$ & Unemployed / disabled & 11.4 \\
\hline Attitude (1-5) & & $3.90 \pm 0.41$ & & \\
\hline Self-efficacy (1-5) & & $3.96 \pm 0.53$ & & \\
\hline Subjective Norm (1-5) & & $3.38 \pm 0.82$ & & \\
\hline $\begin{array}{l}\text { Motivation to be physically active } \\
(1-10)\end{array}$ & & $7.66 \pm 1.43$ & & \\
\hline
\end{tabular}

As regards the sitting time/PA time subgroups, the subjects with low sitting time and sufficient PA had the lowest BMI, while the respondents with high sitting time and sufficient PA time had the highest BMI. The difference between these groups was statistically significant ( $p=0.03$; Cohen's $d=0.42$ ) (Table 4.2). 
Table 4.2: Mean $( \pm S D)$ body mass index $\left(\mathrm{kg} / \mathrm{m}^{2}\right)$ by categories combining total sitting time and total physical activity time.

\begin{tabular}{lcc}
\hline & BMI & BMI >30 (\%) \\
\hline Low total sitting time / sufficient PA time ( $\mathrm{N}=54)$ & $30.3 \pm 4.0 *$ & 57.4 \\
Low total sitting time / insufficient PA time ( $\mathrm{N}=42)$ & $31.1 \pm 4.7$ & 57.1 \\
High total sitting time / sufficient PA time ( $\mathrm{N}=58)$ & $32.4 \pm 5.9$ & 65.5 \\
High total sitting time / insufficient PA time (N=67) & $31.7 \pm 5.0$ & 61.2 \\
\hline
\end{tabular}

${ }^{*} p<0.05$ different from high total sitting time / sufficient PA time; $P A=$ physical activity

Correlation analyses showed that more highly educated people and those who were employed had a higher sitting time, while homemakers had a lower sitting time. Respondents with higher sitting times were less physically active. Higher BMI levels were more prevalent among the younger age group, low-educated respondents, those in employment, respondents from non-Western countries and those with a high sitting time (but not a high PA time). Weight status was also positively correlated with outcome expectancies and subjective norm (Table 4.3).

Linear regression analyses showed that a higher BMI was negatively associated with age and education. Younger participants had a higher BMI than older participants. Respondents with a medium-level or high education had a lower BMI than those with a lower education (Table 4.4).

Logistic regression showed that the BMI groups were associated with age, self- efficacy and sitting time. Younger respondents were more likely to be in the obese group than older ones. Respondents in the lower BMI group had higher self-efficacy and spent less time sitting than obese subjects (Table 4.4).

Linear regressions with the motivation to be physically active showed that people who had a medium-level education were more motivated than the lower educated respondents. A higher motivation was associated with higher self-efficacy. Obese respondents were more motivated to be physically active than those in the lower BMI group (see Table 4.4). 
Table 4.3: Correlates of the demographic variables, cognitive factors, behavioural factors and weight status

\begin{tabular}{|c|c|c|c|c|c|c|c|c|c|c|c|c|c|c|c|c|c|}
\hline & \multicolumn{9}{|c|}{ Demographic variables } & \multirow{3}{*}{$\begin{array}{l}\text { Country } \\
\text { of birth }\end{array}$} & \multicolumn{4}{|c|}{ Cognitive factors } & \multicolumn{2}{|c|}{ Behavioural factors } & \multirow{3}{*}{$\begin{array}{l}\text { Weight } \\
\text { status }\end{array}$} \\
\hline & \multirow{2}{*}{ Gender $^{a}$} & \multirow{2}{*}{ Age } & \multicolumn{3}{|c|}{ Education } & \multicolumn{4}{|c|}{ Occupation } & & \multirow{2}{*}{$\begin{array}{l}\text { Outcome } \\
\text { expec- } \\
\text { tancies }\end{array}$} & \multirow{2}{*}{ Attitude } & \multirow{2}{*}{$\begin{array}{c}\text { Self } \\
\text { efficacy }\end{array}$} & \multirow{2}{*}{$\begin{array}{c}\text { Subjec- } \\
\text { tive } \\
\text { norm }\end{array}$} & \multirow{2}{*}{$\begin{array}{c}\text { Total } \\
\text { sitting } \\
\text { time }\end{array}$} & \multirow{2}{*}{$\begin{array}{c}\text { Total } \\
\text { PA } \\
\text { time }\end{array}$} & \\
\hline & & & Low & Medium & High & Working & $\begin{array}{l}\text { House- } \\
\text { wife }\end{array}$ & Retired & $\begin{array}{c}\text { Un- } \\
\text { employed }\end{array}$ & & & & & & & & \\
\hline $\begin{array}{l}\text { Outcome } \\
\text { expectancies }\end{array}$ & 0.06 & $-0.20^{* *}$ & -0.06 & 0.04 & 0.02 & $0.21^{* *}$ & 0.01 & -0.04 & -0.04 & 0.04 & & & & & & & \\
\hline Attitude & 0.06 & 0.01 & 0.12 & -0.09 & -0.05 & 0.01 & 0.02 & -0.13 & -0.13 & 0.00 & $0.31^{* *}$ & & & & & & \\
\hline Self efficacy & -0.01 & -0.03 & -0.05 & 0.07 & -0.02 & 0.01 & 0.08 & -0.10 & -0.10 & -0.01 & $0.51^{* *}$ & $0.36 * *$ & & & & & \\
\hline $\begin{array}{l}\text { Subjective } \\
\text { norm }\end{array}$ & 0.00 & -0.06 & 0.02 & -0.07 & 0.06 & 0.11 & 0.02 & -0.08 & -0.07 & 0.12 & $0.23^{* *}$ & $0.25 * *$ & $0.21^{* *}$ & & & & \\
\hline $\begin{array}{l}\text { Total sitting } \\
\text { time }\end{array}$ & $-0.17^{*}$ & -0.09 & -0.09 & -0.08 & $0.19 * *$ & $0.15^{*}$ & $-0.18^{* *}$ & -0.00 & -0.00 & -0.04 & -0.05 & -0.03 & -0.04 & 0.03 & & & \\
\hline $\begin{array}{l}\text { Total PA } \\
\text { time }\end{array}$ & -0.09 & -0.05 & 0.03 & -0.06 & 0.03 & 0.12 & -0.08 & 0.06 & 0.06 & -0.09 & 0.05 & 0.04 & -0.07 & 0.02 & $-0.17^{*}$ & & \\
\hline Obesity & 0.11 & $-0.21 * *$ & $0.15 *$ & -0.11 & -0.06 & 0.13 & 0.07 & 0.01 & 0.02 & 0.10 & $0.16^{*}$ & $0.15^{*}$ & -0.01 & 0.13 & 0.10 & 0.01 & \\
\hline BMI & 0.09 & $-0.33^{* *}$ & $0.18^{* *}$ & -0.08 & $-0.14 *$ & $0.14^{*}$ & 0.03 & 0.05 & 0.07 & $0.13^{*}$ & $0.16^{*}$ & 0.08 & 0.01 & $0.13^{*}$ & $0.13^{*}$ & -0.04 & $0.74^{* *}$ \\
\hline
\end{tabular}

Male (1); female (2), ${ }^{b}$ Netherlands (0); other country(1) ${ }^{c} B M I<30$ (0); BMI > 30 (1)

* correlation is significant at the 0.05 level ** correlation is significant at the 0.01 level 
Table 4.4: Linear regression analyses predicting Body Mass Index (BMI) and motivation to be physically active, and logistic regressions predicting obesity.

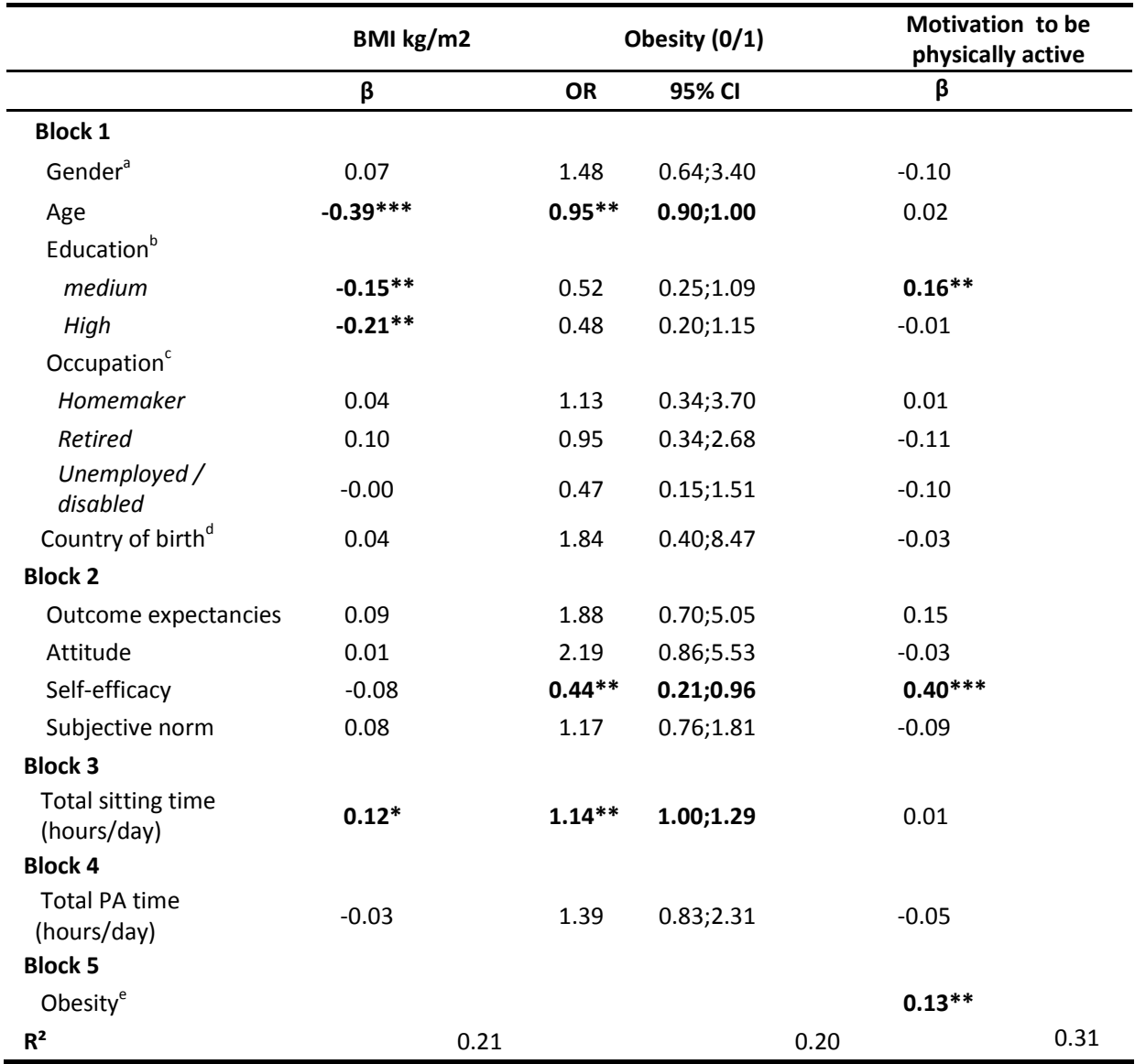

$\bar{a}$ Male (1); female (2), ${ }^{b}$ reference category = low education, ${ }^{c}$ reference category $=$ employed ${ }^{d}$ Netherlands (0), other country(1)

${ }^{e} B M I<30(0) ; B M I>30(1)$

$* * *<0.01, * *<0.05, *<0.10$

\section{Discussion}

The results for this specific group of diabetic or pre-diabetic patients with overweight or obesity showed that the total sitting time was more closely related to BMI than total PA time. Subjects with high sitting time had a higher BMI, even after correction for total PA time. This is in line with the results of Santos and colleagues [93] who demonstrated that, after adjusting for potential confounders (including total PA time) total sitting time was positively associated with BMI. In fact, the differences between 
the groups in our study were even larger than the differences demonstrated by Santos and colleagues. Furthermore, our results showed that subjects with high total sitting time and sufficient total PA time had the highest BMI, and that, after adjustment for potential confounders (including Total PA time and cognitive determinants of being physically active), sitting time was positively associated with being obese. We therefore conclude that even those diabetics or pre-diabetics who meet public health guidelines for leisure time PA may be at risk for co-morbidities if they spend a large proportion of their residual leisure time in sedentary activities. These results indicate that it is important in future lifestyle interventions to aim not only at increasing PA but also at decreasing total sitting time.

Because of the key role NEAT plays with obese people, it will be beneficial if sedentary participants of lifestyle interventions will succeed in decreasing their sedentary behaviour. Sedentary behaviour has been shown to be a risk factor for type 2 diabetes and increased mortality [104]. Various studies have investigated the effects of television viewing on BMI in type 2 diabetes patients [104-107]. In line with our results, Dunstan and colleagues showed that even when people met the exercise guidelines, television viewing was associated with weight gain [106]. Another study showed that women who spent a considerable amount of time watching television had an increased risk of type 2 diabetes at every level of PA [107]. A similar relationship was found in a study with men [105], where men who were obese and physically active had a substantially increased risk for type 2 diabetes compared with those who were lean and inactive, while obese and inactive men were at highest risk [105].

Although our selection method meant that all subjects in our sample were motivated to be physically active, our results indicated that subjects with a higher BMI were more motivated and demonstrated a more positive attitude towards participating in a lifestyle intervention than the subjects with a lower BMI. However, obese subjects also had lower self-efficacy regarding successful participation in lifestyle interventions compared to those with a lower BMI. This is in line with the results discussed in a review by Allen [98], who reported that different exercise studies with diabetes patients had found a significant relationship between self-efficacy and exercise behaviour [98]. A study by Plotnikoff and colleagues [100] also reported that self-efficacy was more important than outcome expectancies in predicting PA; their results supported the need to target self-efficacy to set goals and change behaviour in PA promotion [100]. Participation in PA programmes has been shown to improve selfefficacy and reduce mental health problems, which can have effects on the quality of life [108].

Various strategies can be used to increase self-efficacy regarding PA, such as goal-setting, providing training and guidance in performing activities, demonstrating the desired behaviour, giving verbal reinforcement and reducing anxiety [109]. Giving feedback by telephone or clinical follow-up is also a strategy to increase self-efficacy [110]. These strategies are all part of the BeweegKuur intervention. Participants in this intervention are guided by an LSA, who has been trained in Motivational Interviewing, based on the principles formulated by Miller and Rollnick [70]. This technique is 
expected to enable LSAs to raise the patients' confidence about increasing their PA and decreasing their sedentary behaviour. Goal-setting is part of the counselling process. Participants receive a logbook, in which they can enter their goals and record the time spent on PA. These goals and reports are then discussed with the LSA. Furthermore, participants can start the intervention in an exercise setting with a physiotherapist, enabling the latter to show the participants that it is safe to be physically active [81].

One limitation of the present study was its cross-sectional, non-controlled design. This implies that we cannot draw any conclusions on the causality of the identified associations. Another limitation was the use of self-report measures of weight, height, PA and sitting time, which can lead to underestimation (weight) and overestimation (PA time) as well as socially desirable answers. In addition, we were unable to make distinctions between sitting time in different settings (e.g., at work or during leisure time) which are likely to vary in terms of control over behaviour change. The best way to avoid this in future studies would be to combine self-reported measurements with objective measurements.

A further limitation was that all participants in this study had voluntarily subscribed to participate in a lifestyle intervention, implying high levels of motivation among the participants. Since they participated in a lifestyle programme, it is possible that the obese subjects were aware of their high weight status and wanted to lose weight by participating in the intervention. Longitudinal measurements are needed to show whether the higher level of motivation results in larger intervention effects in the obese subjects. Finally, the small sample size was a limitation. Therefore, separate analyses for gender and age groups could not be conducted. Strengths of the study include the relatively large sample of diabetic or pre-diabetic patients and the use of social-cognitive factors in explaining weight status.

\section{Conclusions}

In conclusion, our results from cross-sectional analyses showed that it was not total PA time but total sitting time which was best associated with BMI among diabetics or prediabetics. Obese diabetics or pre-diabetics had higher sitting time, but were also more motivated and had a more positive attitude towards participating in a lifestyle intervention, compared to non-obese participants. But these subjects also had a lower self-efficacy regarding successful participation in a lifestyle intervention. Lifestyle interventions should not only aim to increase total PA time, but also to reduce the total sitting time, to decrease the risk of obesity and type 2 diabetes 


\section{Chapter 5}

Baseline predictors of maintenance of intervention-induced changes in physical activity and sitting time among diabetic and prediabetic patients: A descriptive case series

\section{Submitted as:}

Judith H.M. Helmink, Jessica S. Gubbels, Femke N. van Brussel-Visser, Nanne K. de Vries \& Stef P.J. Kremers. Baseline predictors of maintenance of intervention-induced changes in physical activity and sitting time among diabetic and pre-diabetic patients, submitted. 


\section{Abstract}

Background: The aim of this study was to explore the predictive value of baseline characteristics with changes in physical activity (PA) and sedentary behaviour among diabetic and pre-diabetic patients participating in a primary care based exercise intervention.

Methods: We used a descriptive case series among diabetic and pre-diabetic patients ( $n=119,50.8 \%$ male, mean age (SD) 65.5 (7.8)). Measurements took place with questionnaires at baseline and two years after the start of the intervention. Predictor variables included demographic factors, Body Mass Index, baseline PA and sitting time, and baseline socio-cognitive profile.

Results: At follow-up, respondents spent more time being physically active than at baseline. For the total group, the average sitting time remained almost unchanged between the two measurements. Further exploration showed that respondents who had relatively high levels of PA at the start of the intervention, increased their total sitting time, while respondents with relatively low levels of PA at the start decreased their sitting time. The socio-cognitive profile did not predict behaviour change. The intervention appeared to be suitable for people with a low-education level.

Conclusion: The finding that the socio-cognitive profile did not predict behaviour change may underline the proposition that decisions to initiate and maintain PA behaviour change are to a large extend non-linear events. 


\section{Introduction}

Type 2 diabetes mellitus is one of the most common chronic diseases worldwide, and its incidence is increasing everywhere [111]. The worldwide prevalence of type 2 diabetes was 171 million in 2000 and is expected to increase to 366 million in 2030 [111]. Benefits to health can be achieved by getting inactive people with diabetes to become more active, which can delay the development of complications in the long term and postpone pharmaceutical treatment $[10,11]$. Being physically active is not only important in the treatment of type 2 diabetes, but also decreases the risk of developing the disease [52], making it an important preventive measure. Several programmes have been developed to decrease the incidence of type 2 diabetes by targeting the pre-diabetic patient population (i.e. people with an impaired fasting glucose) $[10,12,21,22]$. In view of the gap between theory and practice, the challenge is to develop effective primary care exercise programmes. In addition, accumulating evidence indicates the importance of reducing sitting time in addition to increasing physical activity (PA) $[18,19,112]$. Therefore, primary care exercise programmes should not only aim to increase total PA time, but also to reduce sedentary behaviour.

A study of five primary care exercise programmes in Denmark found that participants had increased their PA levels after the intervention. The results of this study suggested that between three and six participants have to enrol in a programme for one to achieve an important increase in PA behaviour [30]. A review by Williams [29] indicated that 17 inactive participants had to start an exercise-on-prescription intervention for one to become active at a moderate intensity level [29]. Furthermore, a review by Garrett and colleagues [31] showed that PA interventions through primary care are more cost-effective than intensive gym-based or instructor-led interventions [31]. However, maintaining intervention-induced behavioural changes remains difficult for many people [29].

Research to identify baseline characteristics that predict intervention-induced behaviour change and maintenance is still in its infancy [113]. Intervention studies have typically focused on process indicators of intervention success (e.g. satisfaction, level of use), while others tried to identify mediating cognitive changes (e.g. attitudes, self-efficacy). Although this information is highly relevant for an understanding of the mechanisms of intervention effects, it does not provide health care professionals with information on the responsiveness of particular risk groups to the intervention. Such information would make the efforts of the health professionals more cost-effective, and would provide intervention designers with feedback on risk groups that may not be responsive to the intervention.

The baseline cognitive profile of intervention participants may be of importance in predicting responsiveness to interventions. Three types of determinants are generally distinguished in behavioural theories: outcome expectancies or attitude, subjective norm and self-efficacy. Outcome expectancies refer to a person's perception that a given behaviour will lead to certain outcomes [43] and attitudes are determined by the beliefs about whether the behaviour will lead to desirable or 
undesirable outcomes [94]. Subjective norms refer to expectations about what people in the social environment want you to do [42]. Finally, self-efficacy (or perceived behavioural control) reflects a person's expectation that he or she can indeed perform the desired behaviour [95]. These cognitions have been successfully applied in research on PA (e.g. [97-100]).

Studies have revealed, however, that it is not cognitions but past behaviour that is the most important factor in predicting behaviour change. One study showed that past behaviour was the strongest predictor of behaviour at follow-up [114, 115]. A study by Plotnikoff [115] showed that although the Theory of Planned Behaviour (TPB) was useful for designing a PA promotion intervention for diabetes patients, the level of PA at six months after the intervention was best predicted by the level of PA at baseline, rather than by the TPB-based cognitions [115].

Another explanation for the increase or decrease in PA may be weight status. A population-based survey in Australia showed that, over a 5-year period, abdominal obesity predicted the decrease in PA for both men and women [116]. This is in line with the outcomes of a review which found a high body mass index (BMI) to be associated with less PA [117]. The study by Lakerveld also revealed that women who had high scores for sitting time at baseline were more likely to have reduced their PA levels after five years [116]. As regards gender differences, women were found to be less active than men [117]. Furthermore, as the age of respondents increases, the level of PA tends to decrease $[117,118]$. Lower educational levels were also associated with less PA [117]. On the other hand, being employed was associated with more PA [118].

In view of all this, our study aimed to examine cognitive profile, PA level, sitting time, weight status and demographics as baseline predictors of the maintenance of intervention-induced changes in terms of PA and sitting time among diabetic and pre-diabetic patients.

\section{Methods}

\section{Participants and design}

Respondents in this study were people who had agreed to participate in a primary care exercise programme called BeweegKuur (see [81] for more information). People with an impaired fasting glucose (fasting glucose value (finger prick) $\geq 5.6$ to $\leq 6.0 \mathrm{mmol} / \mathrm{L}$ or fasting plasma glucose value $\geq 6.1$ to $<6.9 \mathrm{mmol} / \mathrm{L}$ ) and persons with type 2 diabetes (according to the 2006 definition; $\mathrm{HbA} 1 \mathrm{c} \geq 7.0$ ) were eligible for inclusion in the intervention. Exclusion criteria were type 2 diabetes combined with three or more complications, serious poly-pharmacy or type 3 hypertension. The aim of the 12 -month intervention is to guide participants with an impaired fasting glucose or type 2 diabetes in achieving a sustained healthy lifestyle [48]. Eligible patients must have an inactive lifestyle (i.e. not meeting the Dutch guideline recommendation of exercising for at least half an hour on five or more days a week) and motivation for behavioural change. The motivation for behavioural changes is subjectively determined by the general practitioner (GP) and lifestyle advisor (LSA). The selection of participants 
differed between the health care professionals; some selected them during consultation, while others actively approached potential participants.

In the BeweegKuur programme, a patient's GP determines whether he or she is eligible for the programme, and patients are referred to a LSA, usually the practice nurse. The LSA provides coaching and supervision, based on principles of Motivational Interviewing [70], and designs an individual exercise programme in close collaboration with the patient. In addition, all participants are referred to a dietician. The GP's practice remains the central location during the BeweegKuur, where patients have frequent contact with the LSA about their progress in the programme and about perceived barriers, with more frequent and intensive appointments during the first three months. The final appointment with the LSA takes place one year after the start of the intervention. After the intervention, participants are expected to go on exercising on their own, using local exercise facilities.

The present study was a descriptive case series, based on the baseline and follow-up measurement data using two questionnaires. The Maastricht University Medical Ethics Committee indicated that its approval was not required for this study. The LSA was responsible for the distribution of the questionnaires to the patients. The participants received the first questionnaire $(t=0)$ at the start of the BeweegKuur intervention. The recruitment period was nine months. The respondents were exposed to the intervention for one year. The second questionnaire $(t=1)$ was sent to the LSA two years after the start of the intervention, after a one-year follow-up period without intervention. Patients completed an informed consent form at baseline. Both questionnaires had to be returned to the university free of postage. A gift voucher was raffled among participants. The baseline questionnaire was completed by 361 participants, 29 of whom could not be reached by their LSA for the second questionnaire. In total, the second measurement was completed by 119 respondents (response rate $35.8 \%$ ). Drop-out analyses showed that the persons who did not complete the second questionnaire did not differ from those who completed both questionnaires, in terms of age, occupation, country of birth, BMI, vigorous-intensity PA time, walking time or total sitting time at baseline. Gender, education, moderateintensity activity and motivation at baseline were significant predictors of response at the second measurement. Men were more likely to fill in the second questionnaire than women $(p=0.04)$. Respondents with a high $(p<0.01)$ or medium-level $(p=0.03)$ education were more likely to fill in the second questionnaire than low-educated respondents. Respondents with a higher level of moderate-intensity activity at baseline $(p=0.04)$ and with a higher motivation at baseline $(p=0.01)$ were more likely to respond to the second questionnaire.

\section{Baseline Measures}

Demographic characteristics, weight, PA behaviour and sitting time

The questionnaire used in this study was designed specifically for the current study, based on validated questionnaires and theory. The scorings method of the domains used in the questionnaire to assess the cognitive profile corresponds with 
recommendations of the primary theoretical frameworks [42, 95]. Demographic variables assessed in the questionnaire were date of birth, gender, educational level (low, medium or high) and occupation. Self-reported weight and height were used to calculate BMI (weight $(\mathrm{kg}) /$ height $(\mathrm{m})^{2}$ ). PA and sitting time were measured with the validated short version of the International Physical Activity Questionnaire (IPAQ) [101], which assessed PA time in a usual week in terms of total time spent on walking and on moderate-intensity and vigorous-intensity PA. Total PA time in was measured by calculating the amount of moderate and vigorous PA time. The proxy measure of sedentary behaviour was the time spent sitting on an ordinary weekday. This was measured with the question: 'During the last 7 days, how much time did you spend sitting on a week day?', where the respondents could fill in the hours or minutes spent sitting. The sitting time includes time spent at work, at home, while doing course work and during leisure time [101]. Activity data were examined for distribution and outliers. Outliers were identified and recoded to the $95^{\text {th }}$ percentile of the distribution.

\section{Socio-cognitive profile}

The questionnaire items regarding the socio-cognitive profile addressed outcome expectancies [43], attitude, subjective norm and self-efficacy towards participating in physical activity intervention [42, 95]. Outcome expectancies were measured with eleven items, with answers on a 5-point scale ('I do not agree at all' (1) to 'I fully agree' (5)). Reliability analysis yielded a Cronbach's alpha of 0.74 . An example of an item is 'If I participate in BeweegKuur, I will lose weight'. The attitude concept was measured on a 5-point scale ('I do not agree at all' (1) to 'I fully agree' (5)) with six items ( $\alpha=0.65)$, for example: 'The BeweegKuur programme suits people like me'. Five self-efficacy questions ( $\alpha=0.83$ ) were asked, using 5-point scales with answering categories ranging from 'I do not agree at all' (1) to 'I fully agree' (5). An example of a question is 'I think I will be able to attend the whole BeweegKuur programme.' The subjective norm was measured with three questions on 5-point scales ('I do not agree at all' (1) to 'I fully agree' (5) ( $\alpha=0.88)$ ). An example of these questions is 'My family wants me to participate in BeweegKuur.' Motivation was assessed by the question 'How motivated are you to be more physically active?' with answering categories on a scale from 0 (not motivated) to 10 (extremely motivated).

\section{Follow-up measures}

The measures that were included to study the maintenance of behavioural changes were PA (vigorous-intensity, moderate-intensity and walking time) and sitting time. The measures were identical to those used in the baseline measurement.

\section{Statistical analyses}

The descriptive statistical analyses were conducted using SPSS 17.0. Frequencies and descriptives were used to assess the background characteristics of the respondents. Shapiro-Wilk tests and sample distribution plots were used to test the normality of the PA measures, total sitting time and BMI at baseline and follow-up, as well as the socio- 
cognitive profile variables at baseline. All variables were non-normally distributed, except for BMI and outcome expectations. Therefore, non-parametric tests were used to further examine the data. Wilcoxon's signed-rank tests were performed to analyze differences in PA measures and total sitting at baseline and at the second measurement.

In addition, linear regression analyses were conducted. For this purpose, we calculated the change scores between baseline (TO) and follow-up (T1) for the following outcome variables: total vigorous-intensity PA time, total moderate-intensity PA time, total walking time and total sitting time. These change scores were used as the dependent variables in the regressions, while independent variables were demographic characteristics (gender, age, education and occupation) as well as the baseline data on BMI, socio-cognitive profile, PA (walking, moderate- and vigorousintensity PA), and total sitting time. Sensitivity analyses were performed to check the robustness of the results. The regression analyses described above where therefore repeated, excluding the respondents below the $5^{\text {th }}$ percentile and above the $95^{\text {th }}$ percentile of the relevant activity change scores. Potential suppressor or mediation effects of PA behaviour in our multivariate equations were checked for using bivariate Spearman correlations of the socio-cognitive profile factors and all outcomes.

Finally, if statistically significant predictors were found, the responsiveness among respondent groups with certain baseline characteristics was explored by means of a median-split analysis for demographics, weight status (non-obese vs obese), socio-cognitive profile, PA and sitting time. Mann-Whitney tests and graphic representations were used to test and interpret the differences between groups. In all analyses, $p$-values $<0.05$ were considered statistically significant.

\section{Results}

Slightly more than half of the respondents were men, and the mean age was 65 years. Almost half of the respondents were low-educated, and $52.2 \%$ had a BMI above 30 (see Table 5.1).

Table 5.1: Descriptive characteristics of the participants $(n=119)^{a}$

\begin{tabular}{|c|c|c|c|c|c|c|}
\hline & $\mathbf{N}$ & $\%$ & Mean \pm SD & & $\mathbf{N}$ & $\%$ \\
\hline Gender & & & & Occupation & & \\
\hline Male & 60 & 50.8 & & Employed & 38 & 31.9 \\
\hline Female & 58 & 49.2 & & Homemaker & 36 & 30.3 \\
\hline Age & 119 & & $65.52 \pm 7.82$ & Retired & 44 & 37.0 \\
\hline Level of education & & & & BMI $\left(\mathrm{kg} / \mathrm{m}^{2}\right)$ & & \\
\hline Low education & 46 & 39.3 & & $<25$ & 9 & 8.0 \\
\hline Medium-level education & 40 & 34.2 & & $25-30$ & 45 & 39.8 \\
\hline \multirow[t]{2}{*}{ High education } & 31 & 26.5 & & $30-35$ & 33 & 29.2 \\
\hline & & & & $>35$ & 26 & 23.0 \\
\hline
\end{tabular}

${ }^{a}$ The number of participants can differ between variables due to missing values 
Median levels of vigorous-intensity PA time increased (with $40.2 \%$ of all respondents actually increasing this behaviour), as did total walking time (with $62.4 \%$ having increased their walking time) (Table 5.2).

Table 5.2: Median and range of respondents' behaviour and socio-cognitive factors

\begin{tabular}{|c|c|c|c|c|}
\hline & $\mathbf{N}$ & $\begin{array}{c}\text { Median (Range) } \\
\text { T0 }\end{array}$ & $\begin{array}{c}\text { Median (Range) } \\
\text { T1 }^{\mathrm{a}}\end{array}$ & $\begin{array}{c}\% \text { increased } \\
\text { time spent in } \\
\text { activity }^{b}\end{array}$ \\
\hline $\begin{array}{l}\text { Total vigorous-intensity PA time } \\
\text { (min/day) }\end{array}$ & 97 & $\begin{array}{c}0.00 \\
(0.00-48.85)\end{array}$ & $\begin{array}{c}0.00 \\
(0.00-161.14)^{*}\end{array}$ & 40.2 \\
\hline $\begin{array}{l}\text { Total moderate-intensity PA time } \\
\text { (min/day) }\end{array}$ & 86 & $\begin{array}{c}12.85 \\
(0.00-106.29)\end{array}$ & $\begin{array}{c}17.14 \\
(0.00-233.57)\end{array}$ & 48.8 \\
\hline Total walking time (min/day) & 85 & $\begin{array}{c}17.14 \\
(0.00-60.00)\end{array}$ & $\begin{array}{c}28.57 \\
(0.00-180.00)^{*}\end{array}$ & 62.4 \\
\hline Total sitting time ( $\mathrm{min} / \mathrm{day})$ & 76 & $\begin{array}{c}360.00 \\
(0.00-720.00)\end{array}$ & $\begin{array}{c}360.00 \\
(120.00-720.00)\end{array}$ & 42.1 \\
\hline Outcome expectancies $(1-5)^{c}$ & 119 & $\begin{array}{c}3.91 \\
(3.00-4.79)\end{array}$ & & \\
\hline Attitude $(1-5)^{c}$ & 119 & $\begin{array}{c}3.83 \\
(3.00-5.00)\end{array}$ & & \\
\hline Self-efficacy $(1-5)^{c}$ & 119 & $\begin{array}{c}4.00 \\
(3.00-5.00)\end{array}$ & & \\
\hline Subjective Norm $(1-5)^{c}$ & 119 & $\begin{array}{c}3.33 \\
(1.00-5.00)\end{array}$ & & \\
\hline
\end{tabular}

${ }^{a}$ Wilcoxon's signed-rank test comparing scores at T0 and T1 * $P<0.001$

${ }^{b} \%$ of respondents of whom the $\Delta T 1-T 0$ scores was $>0$

${ }^{c}$ Score of 3 is a neutral score, a score $>3$ is a positive score and a score $<3$ is a negative score

Moderate-intensity PA time showed a borderline significant increase $(p=0.051)$, with $48.8 \%$ of the respondents having increased this behaviour at the second measurement. Total sitting time remained stable at the follow-up measurement (Table 5.2).

Linear regression analyses (Table 5.3) showed that a greater change in vigorous-intensity PA time between the baseline and follow-up measurements was negatively associated with education level and BMI at baseline. Highly educated respondents and those with a lower BMI had a smaller change in vigorous-intensity PA time between baseline and follow-up than low-educated respondents and respondents with a high $\mathrm{BMI}$ at baseline.

Linear regression also showed that the change in moderate-intensity PA time was predicted by vigorous- and moderate-intensity PA time at baseline. More vigorous-intensity PA and less moderate-intensity PA time at baseline were associated with a greater change in moderate-intensity PA time.

The change in walking time was predicted by age, total sitting time at baseline and $\mathrm{BMI}$ at baseline. Younger respondents had a larger difference in walking time than older respondents. Furthermore, respondents with a high score for sitting time at 
baseline and those with a lower BMI at baseline had a greater change in walking time than those with low score for sitting time and a high BMI at baseline.

Finally, the difference in total sitting time was negatively associated with total sitting time and outcome expectancies at baseline, and positively associated with BMI at baseline (see table 5.3). With the exception of the predictive value of baseline outcome expectancies for changes in sitting time, the socio-cognitive profile did not predict changes in PA behaviour or sitting time.

The sensitivity analyses testing the robustness of the results found in the regression analyses showed no major deviances from the original standardized regression coefficients, although most coefficients became non-significant due to the decreased statistical power because of the smaller sample size. Potential suppressor or mediation effects of PA behaviour in our multivariate equations were checked for by analysing bivariate correlations between the factors of the socio-cognitive profile and all outcomes. These Spearman correlations showed a range of -0.16 to 0.17 (mean 0.02), all non-significant. Regression analyses without the baseline PA behaviour and sitting time showed that there was no predictor effect of the socio-cognitive profile. Even the predictive effect of outcome expectancies on the sitting behaviour became non-significant in those analyses.

To assist the interpretation of the statistically significant regression results, figures were drawn using cut-offs based on the median baseline score for vigorousintensity PA time (median score=0), moderate-intensity PA time (median score=12.85), sitting time (median score=360) and outcome expectancies (median score=3.91), as well as on BMI (non-obese vs obese) and education (low, intermediate, high). These figures showed that all subgroups had a tendency to show favourable changes two years after baseline (graphically represented by the green colour of the lines). However, some groups were found to have increased their sitting time: without exception, these were the groups with relatively favourable behavioural characteristics at baseline (i.e. high vigorous-intensity PA, high moderate-intensity PA, and low levels of sitting time). The subgroup with relatively high levels of outcome expectancies had also increased their sitting time. The groups that were distinguished on the basis of baseline moderate-intensity PA differed significantly in terms of the increase in moderate- intensity PA $(p<.05)$. The groups with low and high scores for sitting time at baseline differed significantly in terms of the difference in sitting time $(p<.001)$ and walking time $(p<.05)$ (see figure 5.1). 
Table 5.3: Linear regression analyses predicting $\Delta$ vigorous-intensity PA time, $\Delta$ moderate-intensity PA time, $\Delta$ walking time and $\Delta$ sitting time.

\begin{tabular}{|c|c|c|c|c|c|c|c|c|c|c|c|c|c|}
\hline & \multicolumn{3}{|c|}{$\Delta$ vigorous-intensity PA time } & \multicolumn{3}{|c|}{$\Delta$ moderate-intensity PA time } & \multicolumn{3}{|c|}{$\Delta$ walking time } & \multicolumn{4}{|c|}{$\Delta$ sitting time } \\
\hline & $\beta$ & $\mathbf{p}$ & $95 \% \mathrm{Cl}$ & $\beta$ & $\mathbf{P}$ & $95 \% \mathrm{Cl}$ & $\beta$ & $\mathbf{P}$ & $95 \% \mathrm{Cl}$ & $\beta$ & p & $95 \% \mathrm{Cl}$ & \\
\hline Gender $^{a}$ & 0.15 & 0.54 & $-30.40 ; 57.19$ & -0.32 & 0.17 & $-107.46 ; 19.42$ & 0.09 & 0.70 & $-37.29 ; 55.38$ & -0.22 & 0.24 & $-193.71 ; 49.63$ & \\
\hline Age & -0.31 & 0.09 & $-3.96 ; 0.29$ & -0.12 & 0.50 & $-4.12 ; 2.06$ & -0.45 & 0.01 & $-5.34 ;-0.79$ & -0.13 & 0.35 & $-8.76 ; 3.19$ & \\
\hline \multicolumn{14}{|l|}{ Education } \\
\hline \multicolumn{14}{|l|}{ Low (reference) } \\
\hline Medium & -0.28 & 0.10 & $-57.21 ; 5.43$ & 0.21 & 0.19 & $-15.83 ; 76.86$ & -0.12 & 0.47 & $-45.97 ; 21.55$ & 0.13 & 0.34 & $-45.85 ; 131.47$ & \\
\hline High & -0.60 & $<0.01$ & $-91.56 ;-18.09$ & -0.03 & 0.87 & $-57.22 ; 48.80$ & -0.36 & 0.06 & $-76.38 ; 2.23$ & 0.14 & 0.38 & $-57.79 ; 148.65$ & \\
\hline \multicolumn{14}{|l|}{ Occupation } \\
\hline \multicolumn{14}{|l|}{ Employed(reference) } \\
\hline Homemaker & -0.39 & 0.15 & $-89.95 ; 13.69$ & 0.33 & 0.20 & $-26.82 ; 125.09$ & -0.27 & 0.27 & $-85.33 ; 24.53$ & 0.19 & 0.35 & $-76.95 ; 211.54$ & \\
\hline Retired & 0.13 & 0.50 & $-24.17 ; 49.11$ & 0.02 & 0.94 & $-52.38 ; 56.67$ & 0.03 & 0.89 & $-36.86 ; 42.27$ & 0.15 & 0.33 & $-53.03 ; 154.78$ & \\
\hline Vigorous-Intensity PA to & 0.00 & 0.98 & $-0.99 ; 1.01$ & 0.30 & 0.03 & $0.15 ; 3.05$ & -0.21 & 0.12 & $-1.91 ; 0.24$ & -0.03 & 0.77 & $-3.22 ; 2.40$ & \\
\hline Moderate-Intensity PA to & -0.07 & 0.65 & $-0.53 ; 0.33$ & -0.46 & $<0.01$ & $-1.70 ;-0.38$ & 0.25 & 0.08 & $-0.06 ; 0.92$ & 0.05 & 0.71 & $-1.05 ; 1.53$ & \\
\hline Walking to & 0.16 & 0.24 & $-0.29 ; 1.14$ & -0.11 & 0.43 & $-1.49 ; 0.64$ & -0.09 & 0.47 & $-1.06 ; 0.50$ & 0.15 & 0.17 & $-0.63 ; 3.44$ & \\
\hline Total sitting time to & 0.06 & 0.70 & $-0.07 ; 0.10$ & -0.16 & 0.29 & $-0.19 ; 0.06$ & 0.30 & 0.04 & $0.00 ; 0.19$ & -0.66 & $<0.01$ & $-0.89 ;-0.41$ & \\
\hline BMI to & -0.35 & 0.01 & $-5.57 ;-0.74$ & -0.07 & 0.59 & $-4.49 ; 2.57$ & -0.34 & 0.01 & $-6.12 ;-0.78$ & 0.23 & 0.04 & $0.37 ; 14.40$ & \\
\hline Motivation to & 0.05 & 0.75 & $-7.79 ; 10.78$ & -0.04 & 0.77 & $-15.58 ; 11.54$ & 0.25 & 0.08 & $-1.26 ; 19.67$ & 0.03 & 0.80 & $-24.05 ; 30.91$ & \\
\hline \multicolumn{14}{|l|}{ Cognitions } \\
\hline Outcome expectancies to & -0.04 & 0.81 & $-42.58 ; 33.27$ & -0.13 & 0.40 & $-79.34 ; 32.10$ & -0.03 & 0.86 & $-44.14 ; 37.03$ & -0.26 & 0.04 & $-221.22 ;-8.08$ & \\
\hline Attitude to & 0.02 & 0.87 & $-35.23 ; 41.38$ & -0.04 & 0.81 & $-66.94 ; 52.88$ & -0.03 & 0.83 & $-45.96 ; 37.27$ & 0.12 & 0.32 & $-54.53 ; 164.03$ & \\
\hline Self-efficacy t0 & -0.00 & 0.99 & $-34.38 ; 33.78$ & -0.01 & 0.95 & $-50.51 ; 47.24$ & -0.02 & 0.90 & $-38.34 ; 33.94$ & -0.06 & 0.64 & $-117.38 ; 72.44$ & \\
\hline Subjective norm to & 0.15 & 0.32 & $-8.33 ; 24.90$ & 0.02 & 0.99 & $-24.53 ; 24.93$ & 0.10 & 0.47 & $-11.38 ; 24.22$ & 0.03 & 0.80 & $-40.71 ; 52.77$ & \\
\hline$R^{2}$ & \multicolumn{6}{|c|}{0.34} & \multicolumn{6}{|c|}{0.43} & 0.61 \\
\hline
\end{tabular}

${ }^{a}$ Male (1); female (2) 
Figure5.1: Graphic representation of changes in PA time, Sitting time, BMI, Outcome expectancies and education
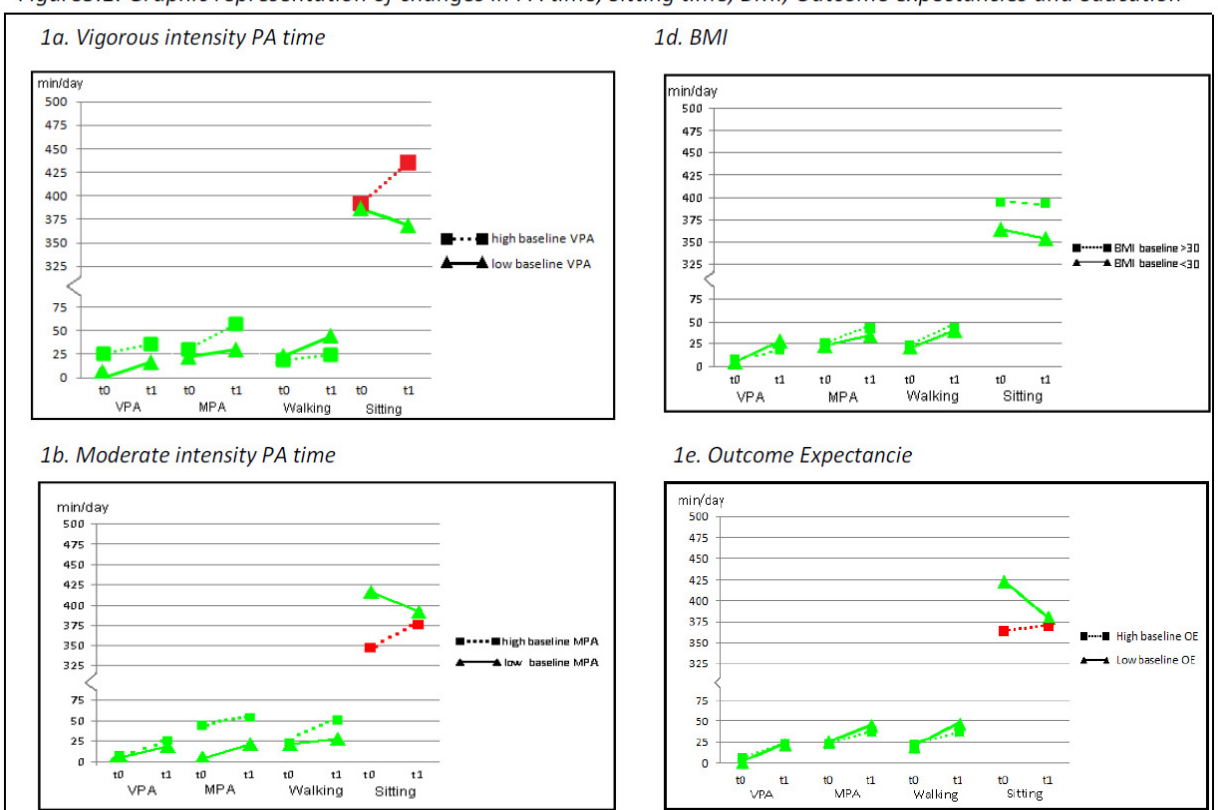

1c. Sitting time

1f. Education
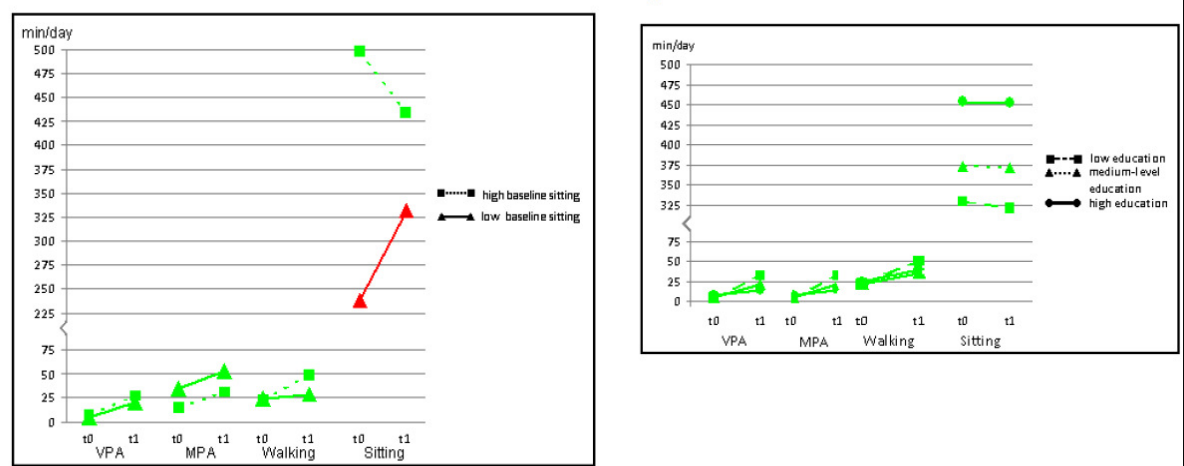

Note: $\quad V P A=$ vigorous-intensity $P A ; M P A=$ moderate-intensity $P A ;$ walking= walking time; sitting=sitting time Green line: desirable behavior; red line: undesirable behavior

\section{Discussion}

On average, respondents in this study were more physically active two years after the start of the intervention than they were at baseline, in terms of each level of intensity (walking and moderate- and vigorous-intensity PA, although the increase was nonsignificant for moderate-intensity PA). The baseline socio-cognitive profile did not predict intervention-induced changes in PA levels. The increase in moderate-intensity 
PA was best predicted by low baseline scores for moderate-intensity PA and high baseline scores for vigorous-intensity PA. The change in sitting time was best predicted by higher scores for sitting time and higher outcome expectancies, and by non-obese weight status at baseline.

The finding that the socio-cognitive profile of the respondents did not predict behavioural outcomes is in line with previous studies $[114,115]$. The limited predictive and mediating power of social cognitions in PA interventions has been the subject of some debate (e.g. $[119,120])$. Whereas social-cognitive theories see behaviour change as a linear, deterministic process, Resnicow and Vaughan [119] argued that decisions to initiate and maintain behaviour change are non-linear events. Behavioural changes may result from a surge of motivation or inspiration that is greater than the sum of its cognitive parts. This is something that happens beyond cognition, represented in statements such as 'It just happened', or 'I just did it'. Our results appear to support this non-linear view of behaviour change. In this respect, our results indicate that GPS or other professionals should not be misled by patients' expressions of high motivation or by perceived low levels of patient motivation or involvement. In fact, expressions of high motivation may reflect highly extrinsic levels of motivation, which are poor predictors of long-term behaviour maintenance [121], while relatively low levels of expressed self-efficacy may reflect a lack of optimistic bias and the inability to set realistic goals. Small units of positive belief structures may in the end lead to 'quantum' changes being made by apparently unmotivated individuals [119, 122]. People may need to cross a 'motivational threshold' to start participating in combined lifestyle interventions, but we would advise intervention designers not to incorporate a high threshold of expressed motivation as a strict inclusion criterion.

Although respondents who scored favourably in terms of the PA measures at baseline increased their scores for PA measures, they also increased their sitting time. Studies have shown that respondents who increased their exercise level compensated this increase with more sedentary behaviour as a strategy to conserve energy [123, 124]. It is therefore important to aim lifestyle interventions not only at increasing PA levels, but also at decreasing sitting time simultaneously. In addition, lifestyle interventions should give more attention to participants' favourable behaviour (i.e. low baseline sitting time), rather than focusing only on behaviours that could be improved.

There was a positive association between baseline sitting time and the change in walking time. Our findings thus indicate that respondents with a high score for sitting time at baseline started with low-intensity activity such as walking. This is in agreement with the (stepped) goals of the BeweegKuur programme, in which participants are encouraged to start with low-threshold activities, such as walking and cycling.

The intervention we studied appeared to be suitable for people with a lower education, and our data shows that the intervention is indeed able to reach these people. Although the differences were non-significant, all outcomes appeared to develop more favourably among low-educated people than more highly educated people. A possible explanation why the BeweegKuur suits respondents with a lower 
education may lie in the referral by GPs. A study by Schmidt and colleagues [37] in the Netherlands showed that referral by GPs was an important motivation to participate, as this is a strong incentive and provides a legitimate reason for starting to exercise. The fact that the BeweegKuur was free of charge for the participants has probably resulted in the relatively large low-SES sample. In addition, the frequent contacts with the LSA, the use of Motivational Interviewing techniques and the tailored BeweegKuur programme may have made the intervention suitable for people with a lower education.

Limitations of the present study were its non-controlled design and the use of self-report measures. The use of self-report measures can lead to underestimation (as regards weight) and overestimation (as regards PA time), as well as to socially desirable answers. The best way to avoid this in future would be to combine selfreported measurements with objective measurements, such as accelerometers to assess activity levels. All respondents in our study had voluntarily agreed to participate in the intervention, which may explain the high average level of motivation. Another point of concern is the high drop-out rate. This could be explained by the fact that participants were approached and reached by the LSA. Questionnaires were sent to the LSA, who had to provide them to the participants. It was impossible for us to detect if all participants in the BeweegKuur were reached with this method. It is possible that some participants did not receive the questionnaire or that they received it at a different time than the researchers indicated. Another limitation of this study was that no process parameters (e.g. attendance to or accomplishment of the programme) were taken into account. Also, there was no measurement immediately after the programme, and we have no information about the respondents' behaviour during the year after the programme. Therefore, the results should be interpreted with caution. Strengths of the study include the relatively large sample of diabetic or prediabetic patients, the longitudinal design, the long-term follow-up and the use of theory-based social-cognitive factors.

The results of this study can be used to inform the development of interventions for this specific target group in future. Interventions may need to put more emphasis on the prevention of increased sitting time. Moreover, acknowledging the possible non-linearity of the relationship between socio-cognitive determinants and behaviour change will help our understanding of this complex and dynamic process. The findings may perhaps also be generalized to other high risk and patient populations, although caution is warranted. Additional research would be needed to examine whether our results would be similar in other risk groups and in other countries.

\section{Conclusion}

To conclude, two years after the start of the intervention, respondents spent more time being physically active. Respondents who had relatively high levels of physical activity at the start of the intervention, increased their total sitting time, while 
respondents with relatively low levels of physical activity at the start decreased their sitting time. The socio-cognitive profile did not predict the behaviour change of the respondents in this sample of diabetic and pre-diabetic patients and the intervention appeared to be suitable for people with a lower education. 


\section{Chapter 6}

The BeweegKuur programme: A qualitative study of promoting and impeding factors for successful implementation of a primary healthcare lifestyle intervention for overweight and obese people

Published as:

Judith H.M. Helmink, Stef P.J. Kremers, Leonieke C. van Boekel, Femke N. van Brussel-Visser, Liesbeth Preller \& Nanne K. de Vries (2012). The BeweegKuur programme: A qualitative study of promoting and impeding factors for successful implementation of a primary healthcare lifestyle intervention for overweight and obese people, Family Practice, 29:i68-i74. 


\section{Abstract}

Background: The aim of the study was to identify promoting and impeding factors for successful implementation of a Dutch primary health care-based lifestyle programme called 'BeweegKuur'. BeweegKuur aims to increase the physical activity and change the diet of people at increased health risk due to overweight or obesity.

Objective: To determine perceived promoting and impeding factors in the implementation of the BeweegKuur programme for overweight and obese people.

Methods: This study consisted of 3 focus group meetings with intervention participants, 15 interviews with health care professionals (HCPs) and 1 focus group session with dieticians. The interviews and focus groups were recorded and transcribed verbatim. The data were analysed with the Nvivo qualitative research software package.

Results: For some intervention participants, the invitation to participate in BeweegKuur came unexpectedly, as they had not been diagnosed with an illness. HCPs were aware of this and took time to explain to participants that the programme was appropriate and safe for them. Participants as well as professionals were generally positive about the feasibility of the programme for overweight and obese people.

Conclusions: HCPs as well as intervention participants were motivated to participate in the programme, and generally indicated that the intervention was in accordance with their needs. The multidisciplinary approach and the combination of physical activity and dietary behaviour change can make the BeweegKuur programme a success if potential impeding factors like those identified in the present study are overcome. 


\section{Introduction}

The number of people who are overweight or obese is increasing in Western societies [2]. In the Netherlands, approximately half of the Dutch adult population was overweight in 2007, and $12 \%$ were obese. Obesity increases the risk of developing cardiovascular diseases, some types of cancer and psychosocial problems $[5,6]$. Weight gain is the result of a positive energy balance, in which energy input through food consumption exceeds energy output, which is partly determined by physical activity [4]. Lifestyle interventions focussing on both physical activity and healthy diet have proved effective in preventing weight-related morbidity in relatively controlled research settings $[10,21]$. Because of the gap between theory and practice, the challenge is to make these interventions suitable for 'real-world' settings [23].

Primary health care has been suggested as a good starting point for lifestyle interventions [28], but successful implementation of lifestyle programmes requires the intervention to be (i) in agreement with patients' wishes and expectations and (ii) compatible with the structure and routines of primary health care. Thus, to make lifestyle interventions effective in primary health care, indepth insight is needed into impeding and promoting factors of successful implementation [125]. A number of characteristics have been argued to be essential for good implementation. These include the characteristics of the innovation itself [39]. For example, does the innovation fit the organization? What are the impeding and promoting contents of the innovation? Also, the characteristics of the user are important [39]. Does the user have a positive attitude towards the intervention and is he or she skilled enough $[125,126]$. The factors which are crucial on the patient level include knowledge, skills, attitude and compliance [127]. Furthermore, the characteristics of the organization are important. In lifestyle interventions, health care professionals (HCPs) work in multidisciplinary teams consisting of professionals from disciplines that have traditionally not been part of the HCPs work-related network. Finally, the characteristics of the social-political context are crucial, such as financial arrangements and policies. This article reports on a qualitative study into the recently developed Dutch primary health care lifestyle intervention called 'BeweegKuur' (Dutch for 'exercise therapy'), examining the crucial implementation issues. The objective of this study is to determine perceived promoting and impeding factors in the implementation of the BeweegKuur programme for obese and overweight people.

\section{Methods}

\section{Background information on BeweegKuur}

In 2008, the Netherlands Institute for Sport and Physical Activity developed BeweegKuur as an evidence and practice-based intervention focussing on both dietary behaviour and physical activity. The aim was an effective and feasible primary health care-based intervention, which in time could be reimbursed under the Dutch basic health insurance scheme (for a detailed description of the development process and intervention contents, including the theoretical and evidence base for the approach, 
see Helmink et al., [81]). Whereas the paper of Helmink et al. [81] describes the systematic process of intervention development, the present study was aimed at a qualitative assessment of the implementation process. The intervention was initially developed for diabetic or pre-diabetic patients, but in 2009 , the programme was further developed for overweight and obese people with a body mass index $>25$ in combination with large waist circumference ( $>88 \mathrm{~cm}$ for women, $>102 \mathrm{~cm}$ for men), as the main selection criteria. Inclusion criteria also allow for people with several types of comorbidity such as diabetes to be included.

The starting point of the BeweegKuur intervention [48] is the GP's practice. The aim of the 12-month intervention is to guide participants in achieving a sustained healthy lifestyle [48] (for a detailed description of the BeweegKuur intervention, see Helmink et al. [81]). The GPs refer patients for an introduction by a so called lifestyle advisor (LSA), normally a practice nurse, who was trained prior to the implementation of the programme to coach and guide patients during the process of initiating and maintaining lifestyle changes. Based on the results of an endurance test, the LSA designs an individual exercise programme in close consultation with the patient and a physiotherapist and the LSA refers the patients to a dietician for individual assessment (see figure 6.1).

Figure 6.1: The pathway of the BeweegKuur intervention

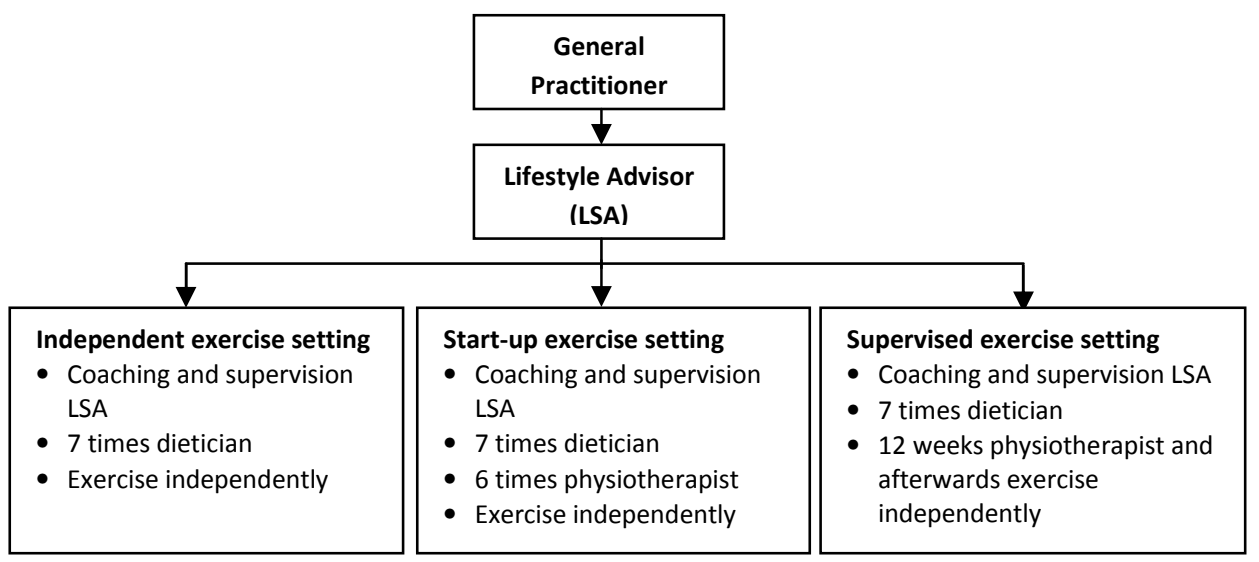

\section{Pilot study}

The BeweegKuur programme for obese and overweight people was implemented at the end of 2009 at five pilot locations across the Netherlands. In total, 36 HCPs started to work with the programme, and 87 patients participated during this period. Qualitative methods were used to assess the opinions of HCPs and patients regarding the programme's feasibility in primary health care practice. Focus group sessions with a total of 16 patients were conducted at 3 locations. Participants were approached by the LSA and they received a gift voucher for their cooperation. Participants filled out an informed consent form and full anonymity was granted. The data were gathered 
between November 2009 and April 2010. All HCPs in the pilot locations were approached by the researcher for an interview or focus group. Due to logistics and time of the HCPs, most of the HCPs were interviewed individually [ $n=15 ; 4$ GPs, 6 physiotherapists ( 2 of whom acted as LSA), 4 practice nurses ( 3 of whom acted as LSA), and 1 dietician who acted as an LSA)]. Three interviews were done by telephone, the others face to face. In addition, a 1-hour focus group session with five dieticians was organized. The point of saturation was reached at the final interview. The interviews and focus groups were recorded and transcribed verbatim with the consent of the participants and transcripts were anonymized. Two researchers (JHMH and LCVB) interviewed the HCPs and led the focus groups and classified the interviews by theme, using the Nvivo qualitative research software package. The content analysis of the transcription was performed by two researchers (JHMH and LCVB). A codebook was developed before the interviews based on the interview scheme and the transcripts were systematically analysed by the principal investigator (JHMH). A second researcher (LCVB) verified the coding and made further suggestions. The themes discussed during the interviews and focus groups are systematically presented in Table 6.1.

Table 6.1: Themes discussed during the focus groups and interviews, per subgroup

\begin{tabular}{|c|c|c|c|}
\hline Theme & $\begin{array}{l}\text { Focus group } \\
\text { with patients }\end{array}$ & $\begin{array}{c}\text { Interviews with } \\
\text { health care } \\
\text { professionals }\end{array}$ & $\begin{array}{c}\text { Focus group } \\
\text { with } \\
\text { dieticians }\end{array}$ \\
\hline Motivation to participate & $\mathrm{X}$ & $\mathrm{X}$ & $\mathrm{X}$ \\
\hline Facilitators and barriers of the programme & $\mathrm{X}$ & $\mathrm{x}$ & $\mathrm{x}$ \\
\hline Content of the programme & $x$ & $x$ & $x$ \\
\hline $\begin{array}{l}\text { Factors determining the success of the } \\
\text { programme }\end{array}$ & $\mathrm{X}$ & $x$ & \\
\hline Dietary programme: group education & $\mathrm{x}$ & $x$ & $\mathrm{x}$ \\
\hline Dietary programme: individual consultations & $X$ & $\mathrm{X}$ & $\mathrm{X}$ \\
\hline Exercise programme & $\mathrm{x}$ & $\mathrm{X}$ & \\
\hline $\begin{array}{l}\text { Combination of physical activity and dietary } \\
\text { behaviour advice }\end{array}$ & $x$ & $\mathrm{x}$ & $x$ \\
\hline Guidance by health care professionals & & $\mathrm{x}$ & $\mathrm{x}$ \\
\hline Financial contribution by participants & $x$ & $x$ & \\
\hline $\begin{array}{l}\text { Differences and/or similarities between diabetes } \\
\text { and obese or overweight participants }\end{array}$ & & $x$ & \\
\hline $\begin{array}{l}\text { Changes in working methods after redefinition } \\
\text { of target group from DM to overweight }\end{array}$ & & $x$ & \\
\hline $\begin{array}{l}\text { Skills required to work with the new target } \\
\text { group }\end{array}$ & & $\mathrm{x}$ & $\mathrm{x}$ \\
\hline Inclusion criteria & & $x$ & $\mathrm{x}$ \\
\hline Refusing to take part in BeweegKuur & & $\mathrm{x}$ & \\
\hline Collaboration by health care professionals & & $x$ & $x$ \\
\hline Maintaining lifestyle changes & $\mathrm{X}$ & $x$ & $x$ \\
\hline
\end{tabular}


To facilitate the interpretation of the data, a thematic framework method has been used [128] and the topics were categorized under the following themes: motivation and barriers for participation, physical activity, dietary behaviour, combination of physical activity and dietary behaviour and maintaining lifestyle changes.

\section{Results}

\section{Motivation and barriers for participation}

The main reasons for participants to take part in BeweegKuur were prevention of diseases and/or complications, losing weight and changing lifestyle. One participant stated 'I want to change my lifestyle; otherwise I'll get diabetes'. Some participants were convinced by the LSA or GP to start the intervention, and for some participants the invitation to participate in the programme was unexpected because they were not aware of being at high risk of overweight-related morbidity as a result of their unhealthy lifestyle.

All HCPs had had 2 years of work experience with the BeweegKuur programme when it was still aimed at diabetic or pre-diabetic patients, but they shared the opinion that the further development of the programme for overweight or obese people was an essential improvement. A practice nurse stated: 'It's the target group that you hope will exercise more. With this programme, you can offer them something'. Another physiotherapist commented: 'The participants in this target group feel that they have failed at different levels, at the level of exercise as well as that of dietary behaviour. It is a benefit to offer these people a programme'. It is a new approach for GPs to identify potential intervention participants based on their overweight status, rather than on the diagnosis of a disease. HCPs noticed some differences between participants with diabetes and those with overweight or obesity. In the perception of HCPs, overweight or obese people were more embarrassed about their physical appearance (a practice nurse: 'Physical appearance plays an important role'), while diabetes patients were more aware of the fact that a healthy lifestyle could improve their health (LSA: 'Diabetic patients are more aware that they can do something about their health'). These professionals thus indicated that the main reason for overweight and obese people to participate was to lose weight, while the diabetic patients generally appeared to participate to improve their health. The professionals reported they needed more time to convince nondiabetic participants that it is good and safe to participate in BeweegKuur, since diabetic patients were already more used to seeing their GP or practice nurse regularly to discuss their lifestyle. A GP stated: 'A diabetic patient is already a patient with a chronic disease and comes four times a year to the GP for a check-up. You talk about lifestyle with these patients more often. An overweight or obese patient is only too heavy and not sick yet. It is not always easy to start and talk about lifestyle and weight with these people'. All HCPs thought it was important that the participants consulted their GP before attending the programme, although in practice this did not always occur due to GPs' 
lack of time. One HCP said: 'I think it's important. It can also be important for the participant to have the GP's support'.

Most HCPs had attended the course on motivational interviewing provided to all professionals involved in the intervention; only some GPs did not attend. The HCPs thought that motivational interviewing was an important skill to motivate patients to participate in BeweegKuur. However, a barrier in this respect was lack of time for optimal use of motivational interviewing techniques. A practice nurse stated "I do not have enough time to apply Motivational Interviewing in the right way'. HCPs stated that one important reason for patients to refuse participation was having to pay for the exercise programmes offered by the physiotherapists, which some were unwilling or unable to do. A physiotherapist said: 'Paying for the exercise programme is a problem in this area. At first, we let them pay to exercise here, but a lot of patients refused to come. Now it is free and more people came'. Finally, time investment by participants or inability to come to meetings during working hours was also reasons to refuse. A LSA stated: 'The people in the overweight and obese group are younger and have to work during the day when the meetings are planned'.

\section{Physical activity}

The focus group participants who had been allocated to the independent exercise setting had started various physical activities, such as walking, cycling, Nordic walking, swimming, and dancing. Participants allocated to the start-up and supervised exercise settings were positive about the physiotherapy programme. A respondent stated: 'The physiotherapist sees everything, even when it doesn't go well'. Supervision by the physiotherapist was perceived as valuable and some participants wanted to stay with the physiotherapist after the three months of training. This option was indeed offered at two locations, at a price which was almost equal to going to a gym. Some participants preferred to exercise alone, while others preferred group exercising. A patient stated: 'I rather exercise in a group, we have a nice group'. Another patient preferred to exercise alone: 'If you go for a walk you can do it whenever you want, whereas in a group you have to be there on time'.

HCPs perceived the three exercise settings to be appropriate for this target group, though they also reported that some participants were disappointed about being allocated to the independent exercise setting. Some participants had expected that the programme would consist of 3 months of training with a physiotherapist, rather than having to exercise on their own. One LSA stated: 'Some patients are enthusiastic because they think they can go to the supervised exercise setting. If we refer them to the independent exercise setting, they're really disappointed'.

\section{Dietary behaviour}

Respondents were satisfied with the dietary programme offered to them. They indicated that it was important that the content and goals of the dietary programme were made clear from the start. Attendance at individual consultations with dieticians 
varied, partly because of costs, the expectation that it would not add much to the group meetings or negative experiences with past visits to a dietician. As one respondent expressed it: 'In the past I went to a dietician and she told me what I was allowed to eat and what not. If you have a consultation with the dietician she focuses on you. Now I am in a group, so the attention is not only focused on me, and for me that works better'. Other respondents thought they would benefit more from individual consultations: "I think that you can make better arrangements with the dietician during an individual consultation than during the group meetings'. The majority of the respondents were willing to participate in the group education meetings. A reason not to attend the meetings was a fear of showing dietary behaviour and emotions in the group. For some respondents, the information in the meetings was not new; nevertheless, they felt that the meetings were useful as a backup to prevent an unhealthy lifestyle. A respondent stated: 'The information in the meetings is not new, but now I have a back up so I will improve my behaviour sooner'. Another perceived advantage of the group meetings was that participants could compare themselves with others. Some respondents had wanted more information about the combination of their diet and their increased exercise behaviour.

Some LSAs were of the opinion that they ought to be present at a patient's first dietary education meeting, to lower the threshold. Since participants already knew the LSA, this could increase their willingness to go. An LSA stated: 'Patients think it is nice if we are there'. It was perceived as important by HCPs that LSAs explained to participants that BeweegKuur consisted of both an exercise and a dietary programme and that they should try and convince participants to attend the first session to see 'that it's not so bad'. Some dieticians saw that participants were very negative at the start, but became more positive during the meetings. A dietician: 'One participant was very skeptical and didn't know what to do before the meeting. During the meeting, she was talking the most'. A disadvantage of the individual consultations is that Dutch health insurance does not cover the full costs. People consulting a dietician have to pay part of the cost themselves, which made some participants decide not to attend individual consultations. A dietician stated: 'The payment really plays a role. One participant cancelled the BeweegKuur when he heard he had to pay'.

Combination of physical activity and dietary behaviour

The respondents thought the exercise programme was the most attractive part of BeweegKuur, although they all regarded the combination of exercise and dietary behaviour as necessary. One respondent said: 'It isn't possible to do the one without the other'. The combination of the exercise and dietary programme was perceived as a considerable burden, especially for those in the supervised exercise setting, and particularly in the beginning.

HCPs were also convinced of the necessity of combining the dietary and exercise programmes. However, most of the dieticians aimed their educational efforts solely at 
improving dietary behaviour. A dietician stated: 'I only aim at an improved diet and not at more physical activity'. Only one dietician took the participants' increased physical activity behaviour into account in their dietary advice.

\section{Maintaining lifestyle changes}

The focus groups revealed that participation in BeweegKuur was perceived as a success by the participants when they lost weight, and that continuation of a healthy lifestyle was also an important aim for the participants. Some respondents doubted whether they would be able to maintain their healthy lifestyle after the programme: 'I think it'll be hard to keep up the healthy lifestyle'.

HCPs commented that it was important that the list of available local exercise facilities was complete and up to date, to sustain lifestyle changes. An important prerequisite for sustained engagement in physical activity is a smooth transition of the participants to local facilities. The five pilot locations had worked with the BeweegKuur programme for almost 2 years, and their lists were now sufficiently complete. During the first year of the programme, however, the list had been incomplete, which had hindered the transition. All professionals intended to discuss the BeweegKuur programme with participants when these patients returned to their practice after completing the programme, in order to follow-up on the process of lifestyle change. Practice nurse: 'I always talk about their lifestyle with the patient when they return at a check up'. A GP stated: 'I only see the participants once a year and there is not always time to talk about the programme. But I always try to talk about lifestyle'.

\section{Discussion}

A new aspect of lifestyle interventions such as BeweegKuur, at least for primary health care in the Netherlands, is that the selection of potential participants is based on risk factors for diseases, rather than on actual diagnosis of a disease. This approach implies a shift towards a more preventive attitude for all professionals concerned. For some participants, the invitation to participate in the programme was unexpected, as they were not diagnosed with a disease. HCPs were aware of this and indicated they needed more time to explain to patients that the programme was appropriate and safe for them. In this study, participants as well as HCPs were generally positive about the feasibility of the programme for overweight and obese people. Referral by a GP was perceived as important by both participants and professionals. A study by Schmidt et al. [37] showed that being motivated by a GP can be important for patients and provides a strong incentive to exercise. Attractive features of our intervention are the multidisciplinary approach and the combination of physical activity and improved dietary behaviour. However, our study also identified some potentially impeding factors for the implementation of BeweegKuur, as outlined below.

An important skill to motivate participants is motivational interviewing, and all HCPs had been trained to use this. Previous studies showed that motivational 
interviewing is effective in coaching lifestyle changes [89, 129-131]. However, these techniques are not easily applied in routine practice and require intensive training and practice support by specific feedback [89]. The fact that motivational interviewing takes more time than traditional counselling techniques could be a barrier for HCPs to implement it in the BeweegKuur programme.

In our study, they did indicate lack of time, as was also reported in other studies $[33,126,132,133]$. The BeweegKuur programme was perceived as a success by the participants when they lost weight or reached their target weight. However, weight reduction results from a consistently negative energy balance over a prolonged period, and exercise will lead to an increase in muscle tissue, resulting in better physical condition but not necessarily in weight reduction for all participants. HCPs in particular should explain this to the participants, and discuss realistic objectives.

The majority of the participants were referred to a dietician. Some respondents had found that dieticians' advice had not helped them in the past, and therefore no longer wanted to consult a dietician. It is crucial that the dieticians' counselling adds to previous experiences and fits in with the BeweegKuur lifestyle approach. This implies, for example, that dietary advice should be adapted to the increased physical activity in the patients' daily routines.

An impeding factor for individual dietary consultations in BeweegKuur was that health insurance did not fully cover the costs. A similar financial impeding factor was identified for the start-up and supervised exercise sessions. Some people were unable or unwilling to pay their share of the cost (a relatively small amount of money; 15 Euros a month) of exercising with a physiotherapist, and in some cases refused to participate in the programme. Some participants were disappointed when they were referred to the independent exercise setting. These respondents had expected that the BeweegKuur involved three months of exercising with a physiotherapist, while in fact they had to exercise on their own, without supervision. The different exercise settings thus need to be explained in more detail to participants before they start. Some participants were not convinced they would be able to maintain their healthy lifestyle after the programme. A complete list of available local exercise facilities was perceived as crucial by the HCPs, as well as giving attention to lifestyle during follow-up meetings after completion of the programme.

Strength of this study is the qualitative study methodology that incorporated both HCPs and patients. The results of this study have been directly used to inform practice; the gathered information has been used to improve the nation-wide implementation of the BeweegKuur in the Netherlands. Some limitations of the present study need to be acknowledged. The same two researchers interviewed and structured the data, which could have introduced bias in the interpretation of the data. Furthermore, the HCPs in this study were selected on the motivation to work with the intervention. Therefore, this selective sample could be more positive about the intervention than their colleagues.

To conclude, both HCPs and participants were motivated to participate in the programme, and they generally indicated the intervention to be in accordance with 
their wishes and needs. If the various potential impeding factors identified in the present study are taken into account, the multidisciplinary approach and the combination of physical activity and dietary behaviour advice can make the BeweegKuur programme a success. 


\section{Chapter 7}

Combined lifestyle interventions in the Netherlands: experiences gained with the BeweegKuur intervention

Submitted (in Dutch) as:

Judith H.M. Helmink, Lieke G.M. Raaijmakers, Geert M. Rutten, Nanne K. de Vries \& Stef P.J. Kremers. Gecombineerde leefstijl interventies in Nederland: ervaringen uit de BeweegKuur, submitted. 
In 2007, the threat of healthcare costs spiralling out of control induced the Dutch Minister of Health, Welfare and Sport, Ab Klink, to propose that costs could be reduced by focusing on prevention. In his 2007-2011 policy framework letter offering a vision on health and prevention, the Minister described the importance of prevention and called it a priority area for health policy. The Minister commissioned the Netherlands Institute for Sport and Physical Activity (Nederlands Instituut voor Sport en Bewegen (NISB)) to develop a prevention programme for diabetes, entitled BeweegKuur (Dutch for 'exercise therapy'). Programme development started in late 2007. BeweegKuur is a combined lifestyle intervention in which participants are referred by a general practitioner (GP) to a lifestyle advisor (usually the practice nurse or a physiotherapist), who assists the participant over a one-year period in achieving a healthier lifestyle. Participants can choose to exercise at their own initiative or can be referred by the lifestyle advisor to a physiotherapy practice, to enter an exercise programme there. At the same time, all participants are referred to a dietician for individual as well as group sessions. The long-term intention was that BeweegKuur and similar Dutch combined lifestyle interventions like Bewegen op Recept, Van Klacht naar Kracht en Big!Move would be covered by the Dutch basic health insurance scheme.

Since the start of its development in 2007, the BeweegKuur intervention has gone through various changes. At first, the intervention mainly focused on exercise, while the dietician sessions were added as a standard part of the intervention later. In 2009, the Ministry recommended that BeweegKuur should be applied earlier in the causal chain, to assist overweight or obese persons. In this same year, the BeweegKuur intervention was implemented at nearly 150 locations across the Netherlands. At this time, the government's aim was still to include the intervention in the Dutch basic health insurance scheme. However, a change of government in 2010 meant that $A b$ Klink, the Minister who had initiated the programme, was replaced by a new Minister of Health, Edith Schippers, who decided on a new policy course, as a result of which the BeweegKuur intervention is currently (2012) not covered by the insurance scheme. The purpose of the present article is to discuss the process of developing, implementing and disseminating the BeweegKuur intervention, as a typical example of a lifestyle intervention in Dutch primary care.

The BeweegKuur intervention has been systematically developed and implemented by NISB since 2007, while Maastricht University has played a role in the iterative process of development, by continually monitoring the process through implementation studies en using the results to provide feedback. The present article is based on the various monitoring studies that were carried out between 2008 and 2012 $[53,134-139]$. BeweegKuur is currently still the subject of effect studies, the results of which are expected to become available in 2013.

Looking back on the 2007-2012 period, it is clear that BeweegKuur has provided momentum for the realization of various processes relating to the dissemination of local combined lifestyle interventions and the further development of these interventions. These primary care processes (including the formation of multidisciplinary teams, training programmes for health care providers and the 
establishment of local networks) are a direct spin-off of the dissemination of the BeweegKuur intervention in the Netherlands. The process of developing, implementing and disseminating the programme has revealed a number of interesting aspects that can be used to inform the development of future combined lifestyle interventions. The introduction of BeweegKuur has been a very costly and time- and energy-consuming process. Much time has been invested by health care providers and also by the Regional Support Structure for Primary Health Care [ROS] and the NISB in starting up the whole process, building networks, developing a 'social map' and supporting participants in achieving a healthier lifestyle. A decisive factor in the success of the intervention at particular locations appears to be the enthusiasm of the health care providers or the ROS advisors involved. The time, funding and energy investments have led to the development of multidisciplinary networks around the participating practices. The contacts with staff at local exercise facilities are particularly important to ensure continuation of the intended behaviour after the actual BeweegKuur intervention has ended. Although a social map showing the local exercise facilities is available in many places, it is important that such maps are kept up to date. This is a dynamic process of constantly monitoring whether sufficient activities are still available and whether they are compatible with the needs of the target group.

The history of the above development started about five years ago, in 2007, when a small and dedicated group of health care providers started the process of introducing BeweegKuur. Despite many initial problems, the participating health care providers retained their enthusiasm, and the BeweegKuur participants reported favourable results. Nevertheless, both the content and the implementation of the intervention proved to leave room for improvement, and NISB has adjusted many of its elements in revised versions. This process of continual optimisation during the interactive initial stages has led to health care providers expressing their satisfaction with the exercise and diet programme [53, 137, 139]. On the other hand, a recent monitoring study [139] suggests that health care providers tend to adapt the intervention to the specific conditions of the practice where it is offered and the requirements of individual patients. One example of this type of adaptation is that of the group sessions with dieticians: at some locations, it turned out to be more effective to have fewer but longer sessions, while other health care providers chose to follow the original BeweegKuur protocol. These group sessions are actually still a subject of some debate. There are considerable differences of opinion among dieticians about such group sessions: some dieticians prefer to use only individual consultations, while others regard the combination of individual and group sessions as very effective. It remains to be seen what form the dietary programme within the BeweegKuur intervention will take in the future, now that advice by dieticians is no longer covered by the Dutch basic health insurance scheme. Future studies will have to show whether this will affect the BeweegKuur dietary programme or its effectiveness. In 2008, multidisciplinary collaboration in the context of lifestyle interventions was still in its infancy. Hence, ROS advisors were engaged to help set up multidisciplinary teams at each location where BeweegKuur was to be introduced. 
During the first year of introduction, these teams consisted mostly of GPs, practice nurses and physiotherapists. One year later, one or more dieticians were added to these teams at all locations, and by 2009 , the multidisciplinary collaboration was clearly beginning to take shape. The health care providers reported that lines of communication had become shorter and they were more easily able to contact each other. The most recent monitoring study among health care providers, held in the autumn of 2011, clearly confirms this picture [139]. Nearly all of the health care providers involved reported that the multidisciplinary collaboration was becoming more successful, and they were generally pleased with the contacts among colleagues.

In recent years, NISB decided to focus on network formation as a valuable instrument to create closer ties between care and sports within the BeweegKuur intervention, in order to ensure the availability of enough exercise facilities for BeweegKuur participants. Ideally, these networks should include not only health care providers but also various other disciplines such as municipal government officials, regional public health services (GGDs), provincial sports councils, welfare workers and local exercise facilities. Close contacts between the various parties are essential to optimize exercise options for each participant. However, the following example reveals that the ties between care and local exercise facilities are not optimal yet. In 2010, NISB started training specialized BeweegKuur exercise instructors (BKIs). It turns out, however, that many health care providers are not aware where these BKIs are available and have therefore referred few patients to them so far [139]. The contacts between these parties can be improved by making better use of various channels of communication (like the BeweegKuur website). Ties between the health care and sports sectors can also be strengthened by the new 'community sports coordinators' that the Ministry of Health, Welfare and Sport intends to invest in over the coming years.

Considerable progress has also been made in recent years as regards the development of a (digital or printed) 'social map' showing all exercise facilities in an area. When the BeweegKuur project started, no such map was available for most of the locations involved. Although municipal directories listed sports facilities, these were generally unsuitable for the target group, as BeweegKuur participants generally prefer to take part in low-threshold activities like walking, cycling and swimming. Evaluation studies held among healthcare providers over the years have shown that the social map of exercise facilities is becoming better and more complete all the time. Nevertheless, some aspects could be further improved: clear agreements need to be made to indicate who is responsible for keeping the social map for a particular area up to date, and the map needs to be critically examined on a regular basis, to see if it is still complete and if the availability of suitable exercise facilities is still sufficient. Regularly asking these questions can help ensure that the availability of facilities remains sufficient, thus increasing the likelihood that BeweegKuur participants will continue to exercise in the longer term.

Two major obstacles to the implementation of the BeweegKuur intervention are the time investments required and the funding of the healthcare providers. In the 
2011 survey, many of the healthcare providers indicated that the fact that BeweegKuur was not going to be covered by the basic health insurance scheme meant that they would no longer be able to invest as much time in carrying out the intervention. They also reported that the time investment was often out of proportion to the results achieved by the intervention. Only one third of the practices that provided BeweegKuur in 2011 indicated their willingness to continue offering the intervention in full, now that it is not going to be covered. Other healthcare providers (21\%) indicated their intention to implement only a limited part of the BeweegKuur elements in future. Some (31\%) indicated that they would be prepared to use a different combined lifestyle intervention or exercise programme, but not BeweegKuur, and $15 \%$ said they intended to cease implementing combined lifestyle interventions altogether. It is highly important that a reliable funding structure is created to ensure the continued use of BeweegKuur. Local-level negotiations with health insurers take time. The role of the municipal authorities in allocating funds available for care and prevention is also an essential part of the funding options for BeweegKuur. There is a risk that health care providers will start to selectively use elements from the BeweegKuur intervention, which may endanger its effectiveness.

It seems plausible that the processes described above would also have come about if the BeweegKuur intervention has not been introduced, but they would probably have taken much more time and effort and would have been more local. The result of a number of years of investing in the BeweegKuur project is that effective networks have developed at various locations across the country, and that a structure is in place that could be used to successfully implement further combined lifestyle interventions (for other target groups). These experiences underline the importance of keeping track of the level and nature of the implementation and 'sustainability' of combined lifestyle interventions and/or exercise interventions in primary care. Primare care in the Netherlands has in recent years started to change its approach from one emphasizing 'care and illness' to one of 'behaviour and health'. The continued implementation of combined lifestyle interventions can stimulate this change, although the experiences gained with BeweegKuur have shown that it will not always be a smooth development.

In the course of the 2007-2012 period, many health care providers have been given a role in the prevention of chronic diseases like diabetes. In addition, these providers have established more intensive and easier contacts, and have managed to reach target groups that had been difficult to reach. Despite these favourable developments, however, the level of political support will continue to play a major part. If politics fails to provide the necessary support for interventions like BeweegKuur, it will probably be difficult to fund them. Only time will tell whether alternative forms of funding will be found to allow continued effective implementation of combined lifestyle interventions in the Netherlands. 


\section{Chapter 8}

General Discussion 
This thesis reports on the implementation process of BeweegKuur, a primary care based lifestyle intervention tailored to the individual needs of participants, focusing on a change in physical activity and dietary behaviour. This chapter summarizes the main findings of the studies, the methodological considerations, implications for practice and implications for further research. It ends with an overall conclusion.

\section{Main findings of the studies}

The BeweegKuur intervention was systematically developed, had a solid theoretical foundation and focused on both individual counselling and environmental facilitation (chapter 2). During the first (pilot) year of the BeweegKuur intervention, in 2008, several of its features remained to be developed. One of these features was access to existing local exercise facilities. In 2008, the network of these exercise facilities was incomplete and most health care providers were insufficiently aware of the available facilities. This is likely to have contributed to relapse by participants in this first pilot year of the BeweegKuur programme.

The results of an evaluation during the first pilot year showed that the health care providers involved were highly motivated to implement and continue the BeweegKuur intervention (Chapter 3). This can be partly attributed to the fact that the health care providers included in that particular study were likely to be innovators and early adopters [39]: they had volunteered to participate in the pilot implementation. The motivation to continue using BeweegKuur was lower among practice nurses than among other health care providers. One explanation may be the role of practice nurses in the intervention. Practice nurses typically assumed the role of the 'lifestyle advisor' (LSA), who played a pivotal role in the intervention, and were responsible for smooth and unequivocal communication within the multidisciplinary team and for the coordination of individual activities within the intervention. The LSA spends more time counselling the participants than the other health care providers. All these factors may have resulted in a higher workload for these respondents..

Another finding of the evaluation study was that social support by colleagues, perceived compatibility and perceived relative advantage of the intervention for providers were associates of the baseline motivation to implement it. The implementation of the intervention depended on the short-term advantages for health care providers, while the longer-term advantages for the participants were less salient at the stage of deciding whether to implement the lifestyle intervention programme. The motivation to continue using BeweegKuur was best predicted by a high baseline self-efficacy and by the type of profession (i.e. not being a practice nurse). In order to ensure sustained implementation of the BeweegKuur intervention, it was important that health care providers felt confident about performing their tasks. Therefore, an intensified focus on skills development (e.g. general lifestyle counselling skills and motivational interviewing skills) was expected to contribute to sustained high-quality implementation of the intervention. 
The results of the studies conducted among BeweegKuur participants yielded some interesting insights (Chapter 4). The baseline data showed that it was not the total physical activity time, but the total sitting time which was more closely related to body mass index (BMI), as it was the respondents with high total sitting time and sufficient total physical activity time who appeared to have the highest BMI. Also, after adjustment for potential confounders, sitting time was positively associated with being obese. Even those diabetic or pre-diabetic patients who meet public health guidelines for leisure time physical activity may be at risk of co-morbidities if they spend a large proportion of their time in sedentary activities. These results indicate that it is important that lifestyle interventions focus not only on increasing physical activity, but also on reducing sedentary behaviour. This finding supports the current increased research focus on the role of sedentary behaviour as a risk factor for chronic diseases, and the lessons that can be learned from it for lifestyle interventions.

The motivation of the participants at baseline was rather high. The selfefficacy of respondents with a relatively low BMI was higher than that of respondents with a higher BMI. Despite the generally high levels of motivation among the obese participants, intervention designers and intermediaries should be aware of such participants' low level of self-efficacy towards being physically active. Different strategies can be used to increase the participants' levels of self-efficacy, for example goal setting, offering verbal reinforcement and giving feedback by telephone or during consultations $[109,110]$. These strategies are all part of the BeweegKuur intervention.

The results of the study among participants two years after the start of the BeweegKuur programme showed that, on average, respondents spent more time being physically active, at each level of intensity (walking, moderate and vigorous) (Chapter 5). However, the total sitting time remained almost equal between the two measurements. Respondents who had relatively high levels of physical activity at baseline increased their sitting time, while respondents with relatively low baseline levels of physical activity decreased their sitting time. In other words, respondents with favourable baseline scores on the physical activity measures increased their physical activity time, but they also increased their total sitting time. Participants gave the impression of compensating the increased time during which they were physically active by also increasing their sedentary behaviour. Lifestyle interventions may thus profit from giving more attention to the participants' favourable behaviour (i.e. low baseline sitting time), rather than focusing only on behaviours that could be improved. The study findings also showed that the baseline socio-cognitive profile did not predict intervention-induced changes in PA levels, and that low-educated people can be reached by an intervention such as BeweegKuur.

Chapter 6 describes the further development and implementation of the prototype BeweegKuur intervention for obese and overweight people. A new aspect of lifestyle interventions such as BeweegKuur, at least for primary health care in the Netherlands, is that the selection of potential participants is based on risk factors for diseases, and not only on an actual diagnosis of a disease. This approach implies a shift towards a more preventive attitude on the part of all professionals involved. For some 
participants, the invitation to participate in the programme came as a surprise, as they had not been diagnosed with a disease. Health care providers were aware of this and indicated they needed more time to explain to participants that the programme was appropriate for them. Participants and providers were generally positive about the feasibility of the programme for overweight and obese people. Health care providers as well as intervention participants were motivated to participate in the programme, and generally indicated that the intervention fitted their needs. Referral by a general practitioner (GP) was perceived as important by both participants and professionals. According to health care providers, attractive features of BeweegKuur were its multidisciplinary approach, the use of motivational interviewing and the combination of physical activity and improved dietary behaviour. On the other hand, several impeding factors were identified. The first was that the costs of the individual dietary consultations were not fully covered by health insurance. Secondly, health care providers reported not having enough time to carry out the BeweegKuur intervention. Thirdly, there was no complete list of local exercise facilities available for the health care providers. And finally, health care providers were found to devote little attention to lifestyle during follow-up consultations with participants after the completion of the programme. The results of this study were used directly to inform practice, in that the information we gathered was used to improve the nation-wide implementation of BeweegKuur in the Netherlands.

Chapter 7 reflects on the development, implementation and dissemination processes of the BeweegKuur intervention in primary care. BeweegKuur has provided momentum to the processes involved in the implementation of combined lifestyle interventions. Other processes within primary care (for example forming multidisciplinary teams, skills training for health care providers and the establishment of local networks) were a direct consequence of the dissemination of the BeweegKuur intervention in the Netherlands. The dissemination process has been quite successful. In 2011, the BeweegKuur intervention was offered at 140 locations throughout the Netherlands. Some remarkable observations were made during the development, implementation and dissemination period. First, the implementation of the programme took a lot of time and energy on the part of the health care providers. Second, in the primary health care centres that implemented BeweegKuur, multidisciplinary networks were formed and the contacts with local exercise facilities improved during the implementation. Although it is plausible that without BeweegKuur, some of these processes would also have come about, they would probably have taken much more time and effort and would have remained more local. The BeweegKuur programme facilitated the formation of local networks and of a structure in which combined lifestyle interventions could be implemented at different locations in the Netherlands. The findings of the BeweegKuur implementation studies showed that it is important to monitoring the level and form of implementation and sustainability of combined lifestyle interventions in primary care. 


\section{Methodological considerations}

\section{Study design}

The data presented in this thesis were obtained in a longitudinal monitoring study among pre-diabetic, diabetic, overweight and obese participants and various health care providers. Since this study was not an effect evaluation study, we did not use a controlled trial or control group design. This implies that the results of the study cannot be interpreted in terms of the effectiveness of BeweegKuur; they only give an indication of the behavioural development within one cohort. The aim of this implementation study was to use longitudinal data to evaluate and improve the implementation of BeweegKuur, rather than testing the effectiveness of the programme.

The various studies used several quantitative and qualitative techniques. Questionnaires were developed for health care providers as well as participants. Furthermore, face-to-face interviews and focus groups were used to examine the opinions of participants and different types of health care providers regarding the BeweegKuur intervention. The combination of different forms of data collection and different target groups (health care providers and participants), and the fact that different types of health care providers (GPs, practice nurses, physiotherapists and dieticians) were involved in the study enabled us to provide a relatively complete overview of the impeding and promoting factors for the implementation of the BeweegKuur intervention.

A limitation of the studies among participants was the measurement of physical activity and sitting time. These measurements were conducted by means of self-report questionnaires, instead of objective measurements. This may have led to socially desirable answers and/or overestimation of physical activity and underestimation of sitting time. However, self-reported questionnaires, such as the short version of the IPAQ [101] (used in our studies), are more practical and less costly than objective measurements. A review of physical activity questionnaires showed that the short version of the IPAQ was appropriate for the dimensions of physical activity it was intended to measure [140]. However, since the short version of the IPAQ does not discriminate between different settings (home, work, transport, recreation and sport), its content validity seems limited [140]. Another concern regarding the measurement of physical activity with a questionnaire is that moderate-intensity activities are difficult to monitor. These activities are often part of daily routine and therefore more difficult to recall [141].

One strength of our studies was its iterative and field-based character. Since the studies in this thesis followed the programme from the beginning, the programme developers and the researchers improved the intervention in close consultation with the research population. The advantage of following the implementation process of an intervention from the start is that impeding factors can be detected in early development phases and improved immediately. 


\section{Dissemination process}

In chapter 2 of this dissertation the development and implementation process of the BeweegKuur was discussed. In this chapter we mentioned that the BeweegKuur was developed with the Intervention Mapping approach. As a consequence, the reader may have expected a chapter on the application of the Intervention Mapping protocol. Because the BeweegKuur was developed by NISB and we were involved in the process after the first draft of the prototype, it was not possible for us to describe this. Furthermore, this dissertation did not describe and evaluate the implementation strategies used by NISB. It would have had added value to evaluate the strategies used to disseminate the BeweegKuur. During the pilot years (in which the data of this dissertation were collected), health care centres were involved in the programme because of their initial motivation to adopt a lifestyle intervention. The use of specific implementation strategies was more important in the phase after the pilot years when the aim was to disseminate the intervention on a nation-wide basis. However, this national dissemination process was not the primary research topic of this dissertation.

\section{Population}

At the start of the implementation period, the BeweegKuur intervention was aimed at pre-diabetic and diabetic patients. In 2009, the target population was changed to obese and overweight people. This shift in the target group meant that BeweegKuur was now applied earlier in the causal chain. Overweight or obesity can lead to diabetes, but the intervention might now also help prevent other diseases (e.g., cardiovascular diseases, obstructive sleep apnea and different forms of cancer).

The BeweegKuur implementation process involved some impeding factors for health care providers, which may have decreased their motivation during the implementation process. The first impeding factor was the fact that the intervention went through certain changes during the pilot period. Some of the health care providers did not realize this at the start of the process. Furthermore, the time investment it required of the health care providers was higher than expected, especially for the LSAs. Some health care providers invested more time than expected to achieve an optimal implementation of the BeweegKuur. Together with the fact that the question whether the BeweegKuur would be covered by the Dutch basic health insurance scheme remained undecided, this meant that the motivation of some health care providers dropped during the implementation of the programme. In Chapter 3 , the results achieved by all health care providers were pooled. It is possible that the results for some subgroups, for example the LSAs, were different than those for the GPs. However, the small sample size made it impossible to detect these differences.

A disadvantage of the study reported on in Chapter 5 was the high dropout rate $(64.2 \%)$ among the participants. This could be explained by the fact that participants were approached by the LSA. Questionnaires were sent to the LSA, who had to distribute them to the participants. It was impossible for us to detect if all participants received a questionnaire. Questionnaires were sent to the LSA, who had to provide them to the participants. It was impossible for us to detect if all participants 
in the BeweegKuur were reached with this method. It is possible that some participants did not receive the questionnaire or that they received it at a different time than the researchers indicated.

\section{Theoretical framework}

Some comments can be made regarding the instruments we used to assess the cognitions in our studies among BeweegKuur participants. Since it would be somewhat awkward to ask a participant who has just voluntarily agreed to participate in a physical activity intervention to indicate his/her intention to be physically active or to start being physically active, we measured the level of motivation of participants to start being physically active, rather than their intention.

Another comment concerns the fact that we measured the concepts of the Theory of Planned Behaviour (TPB) and Social Cognitive Theory by questions about participants being physically active in the BeweegKuur context, rather than about being physically active regardless of the intervention. Therefore, our questionnaire did not measure cognitions about physical activity behaviour in general, but cognitions about physical activity behaviour in the specific context of the intervention.

The longitudinal study among BeweegKuur participants (Chapter 5) showed that cognitions did not predict the level of physical activity and sitting behaviour at the second measurement. Other theories than the ones we used could be more appropriate to predict physical activity behaviour change. Quality or type of motivation can be a better predictor of physical activity and sitting behaviour than quantitative socio-cognitive measures. One theory that distinguishes different forms of motivation is the Self Determination Theory (SDT). In this theory, motivation lies on a continuum from 'extrinsic' to 'intrinsic' [65]. The SDT presumes that intrinsic motivation and more internalized forms of extrinsic motivation (e.g. being active because people aim for the health benefits of exercise) are associated with more pleasure in exercise, more commitment and a stronger intention to exercise more often [65], in comparison with amotivation or less internalized forms of extrinsic motivation (e.g. being active because of reasons outside the individual). More intrinsic motivation and more internalized forms of extrinsic motivation may result in a person starting to exercise more intensively of their own free will, which increases the likelihood of sustained physical activity behaviour. Additionally, SDT predicts that supporting a person's autonomy, competence and relatedness will improve the intrinsic motivation and the more internalized forms of extrinsic motivation. Long-lasting positive behavioural outcomes require a person's motivation to shift from extrinsic to intrinsic [142]. The SDT has been successfully applied in studies on physical activity (e.g. [143-146]) and weight control (e.g. [147-149]). Future studies that incorporate SDT measures in predicting lifestyle intervention-induced behaviour change may find predictive value in personal motivational profiles.

The limited predictive and mediating power of social cognitions in physical activity interventions has been debated (e.g. [119, 120]). Whereas social-cognitive theories see behaviour change as a linear deterministic process, Resnicow and 
Vaughan [119] proposed to view behaviour change as a non-linear event. Behaviour change results from a surge of motivation or inspiration that is greater than the sum of its cognitive parts. It is something that may occur beyond cognition, as reflected in statements such as 'it just happened' or 'I just did.' Such apparently unpredictable or chaotic processes are the result of the operation of dynamic systems. A unique feature of the dynamic-systems explanation is the underlying assumption that behaviour is not determined by intrinsic central blueprints (e.g., genes) or by extrinsic facilitators (e.g., culture), but rather by the interplay among the elements of the system itself [150]. A consequence of such interplay is that simple elements can give rise to highly unpredictable nonlinear behavioural transitions.

Linear and chaotic paradigms are not necessarily mutually exclusive. Behaviour change includes both chaotic and rational processes. The Cognitive-Planned and Chaotic-Quantum aspects of motivation can be placed along a continuum, in line with the approach adopted by dual-process models. The anchors of the continuum reflect the 'duality' invoked by these models [151]. On the one hand, people can spend large amounts of time, effort and cognitive energy in systematically developing beliefs and decisions. They may, for example, make extensive plans to visit a fitness centre and act exactly in accordance with these intentions. On the other hand, people can also engage in a particular act without utilizing cognitive effort. Behaviour is then the result of direct automatic, chaotic or nonconscious responses to environmental cues $[152,153]$.

\section{Implications for practice}

The findings from the studies presented in this thesis have several implications for practice, which are related to the content of the BeweegKuur intervention, the implementation of combined lifestyle interventions in practice and the context of the intervention.

\section{The content of BeweegKuur}

After the pilot years, health care providers were satisfied with the physical activity and dietary programmes included in the BeweegKuur intervention. In some practices, the BeweegKuur was, as Rogers [39] termed it, 'reinvented' to better fit the organization. For example, the dietary group meetings were redesigned to fit the needs of some dieticians and participants. Instead of eight dietary group meetings each lasting one hour, some dieticians changed this to five 90-minute meetings. Such reinvention was also seen in the physical activity programme. For example, participants at some locations did not train with a physiotherapist three times a week for an hour, but two times a week for 90 minutes. When reinvention takes place, it is conceivable that some of the elements of BeweegKuur are implemented in a different way than intended by the intervention developers. It is also possible that health care providers implement some elements of the BeweegKuur, while ignoring others. This 'cherry-picking' approach may mean that the effectiveness of the BeweegKuur intervention is no 
longer assured. However, when the health care providers do not adjust the BeweegKuur intervention to their needs, there is a risk that they will stop using the intervention, because of the mismatch between intervention and health care practice.

The data of our studies indicated that not only the increase of physical activity is important to gain health benefits, but also the decrease of sedentary behaviour. The BeweegKuur is aimed at the increase of physical activity level of the participants. LSAs have the role to develop an exercise programme for the participant. This can be exercising on an intensive level or on a low level, for example going to the shop by bike instead of by car. Our studies indicated that decreasing sedentary behaviour can also result in health benefits. Therefore, lifestyle interventions such as the BeweegKuur should promote the low intensity activities to decrease the sedentary lifestyle in addition to the promotion of higher levels of high intensity physical activity.

One of the promising aspects of BeweegKuur is the use of motivational interviewing. All health care providers involved in the BeweegKuur programme were invited to attend a course on motivational interviewing. However, these techniques are not easily applied in routine practice, and they require intensive training and support by specific feedback [89]. Studies have found that training alone is insufficient to maintain the skills needed for motivational interviewing [154, 155]. Good and effective motivational interviewing requires on-going training, feedback and coaching [154, 155]. A barrier for health care providers to implement and continue using BeweegKuur can be the fact that motivational interviewing takes more time than traditional counselling techniques. However, the advantage of motivational interviewing is that its goal is to increase a participant's intrinsic motivation, rather than relying on the more extrinsic forms of motivation [156]. Motivational interviewing assumes that clients themselves know what is best for them, and that counsellors are there to guide them on their way to behaviour change [156]. Motivational interviewing uses strategies that are more supportive than argumentative and more persuasive than coercive, and it offers a particular method to help a person do something about their unhealthy lifestyle [156].

Our studies did not determine the extent to which or the exact way in which motivational interviewing was applied by the health care providers. Some of them indicated during the pilot period that they needed further coaching or training in motivational interviewing for optimal use. It is important that health care providers who work with motivational interviewing unlearn former habits that are less suitable for motivational interviewing, such as the tendency of counsellors to use closed, rather than open-ended questions. It is possible that the motivational interviewing training offered to the providers in our study was too brief to unlearn these habits. Thus, ongoing training and coaching of health care providers is needed for optimal application of motivational interviewing in routine care [157]. Future research will need to devote special attention to the use of motivational interviewing techniques in the counselling practice.

Another important aspect of the BeweegKuur intervention was the level of motivation among participants. The BeweegKuur protocol stated that only participants 
who were motivated to achieve behavioural change should be allowed to join the intervention. It was up to the GPs or LSAs to estimate whether the participants were sufficiently motivated. Our results showed, however, that baseline motivation did not predict the level of physical activity or sitting time one year after the intervention. The variation in the level of motivation was not large enough for it to have predictive value. On the other hand, we feel that GPs could have been misled by participants' expressions of high motivation or by perceived lower levels of participants' motivation or involvement. In fact, expressions of high motivation may reflect highly extrinsic levels of motivation, which are poor predictors of long-term behaviour maintenance [121]. A motivational threshold may be needed for participation in combined lifestyle interventions, but we would advise intervention designers not to incorporate a high level of expressed motivation as a strict inclusion criterion.

Finally, continuity of care was an important issue in BeweegKuur. At the start of the programme, when it was only being applied among diabetes patients, this continuity of care was secured. In the Netherlands, diabetes patients consult their GP or practice nurse every three months, so all patients who started the programme had their regular check-ups. Lifestyle is typically one of the topics at these consultations. However, because not all GPs and practice nurses were involved in the application of BeweegKuur, the practice information system should generate a reminder that a particular pre-diabetic or diabetic patient is a BeweegKuur participant before the patient enters the room for a regular appointment. Since the target group was changed to include overweight and obese subjects, this continuity of care has not been secured in such a systematic manner. Until now, there is no effective system for continuity of care for obese or overweight people, which makes it a challenge for future interventions.

\section{Implementation of lifestyle interventions in primary care}

Our implementation study on BeweegKuur showed that political decisions can have a crucial influence on the continued implementation and dissemination of a lifestyle intervention. In the development phase of the BeweegKuur intervention, the political climate regarding prevention of chronic diseases in primary care was favourable. Therefore, the BeweegKuur developers aimed to have it included in the Dutch basic health insurance scheme by 2012, which meant that the costs of BeweegKuur would be covered. When a new government was installed, whose focus was not so much on prevention, the financial future became uncertain, as a result of which the BeweegKuur intervention was no longer applied at three-quarters of the locations. Only one-third of the primary health care providers who were using BeweegKuur in 2011 expressed the intention to continue using all of its elements in 2012. Almost a quarter of the professionals (21\%) indicated their intention to continue with a limited part of the BeweegKuur elements, while $31 \%$ wanted to switch to another combined lifestyle intervention and $15 \%$ intended to cease implementing combined lifestyle interventions altogether [139]. 
Our study also showed that it took the health care providers a lot of time and energy to use the intervention. Intervention designers should be aware of the amounts of time and energy that health care providers may be willing to invest. Skills training and training to work with the programme can help increase their motivation and willingness to implement an intervention. First of all, training health care providers can help increase their self-efficacy regarding the implementation of a lifestyle intervention. It is also important to train health care providers in the correct way to use the protocol and implement the intervention as intended. Effective training and educational materials could overcome the barrier of lack of time for health care providers [33]. The financial aspect is also important for the implementation and continuation of a lifestyle intervention, as it may become endangered when the costs are too high or when financial benefits are not expected to materialize in the short term.

A study of Linmans and colleagues [158], executed in an early stage of pilot implementation, showed the importance of executing implementation such as those presented in this dissertation. In the study of Linmans and colleagues, pre-diabetes and diabetes patients participating in the BeweegKuur in four health care centres in Eindhoven were compared to pre-diabetes and diabetes patients who received usual care. These patients started in 2008 during the first pilot year of the study. The results of that study showed that the effects the BeweegKuur were small and not significant [158]. However, during the first year when the patients started with the intervention, the conditions of the BeweegKuur were not optimal. Some points needed improvement, such as the fact that the exercise settings and the consultation with the LSA in these settings were still subject to change, and the map with local exercise facilities was still incomplete. Our study during the first pilot year exposed some impeding factors of the BeweegKuur and NISB adjusted the prototype accordingly. Therefore, it is possible that the effectiveness of the BeweegKuur would be different after a few years working with the programme, compared to the study done by Linmans and colleagues [158] in the first pilot year.

Contextual factors in the implementation of a lifestyle intervention

An effective intervention and motivated health care providers and participants are not all that is needed to make a lifestyle intervention a success. One of the most important principles of BeweegKuur was the multidisciplinary collaboration between different health care providers, and between primary health care providers and local exercise facilitators. If there is no collaboration, the implementation of the intervention will be unsuccessful. Forming an effective multidisciplinary team requires guidance by a mediator. In the BeweegKuur programme this was done by NISB or ROS advisors. A team including a range of health care providers was formed at each location, and whenever there were questions, the NISB or ROS advisor was available to help. It is important to constantly and critically evaluate the actions of the multidisciplinary team to ensure optimal implementation of the programme. 
In addition to the multidisciplinary teams, networks of local and regional partners should be formed, which should include local politicians, employees of public health organizations and sport and exercise coaches. This network should produce a map showing local exercise facilities, which is one of the most important factors in promoting the continuation of the participants' physical activity level. During the process of implementing BeweegKuur, it became clear that such maps were not always up to date. Regional or local networks should agree who is responsible for the development and updating of the map. If there is no agreement, it is likely that there will be no map or an incomplete one. Thus, future lifestyle interventions should give special attention to the development of such a map.

The collaboration between the different parties that is required to develop the map of local exercise facilities requires all members of the network to trust each other and to believe that the others are capable of doing their job. If there is no trust, the collaboration between the network members is doomed and the development of the map will be at risk.

\section{Implications for further research}

Future research should investigate the utility, effectiveness and cost-effectiveness of the BeweegKuur intervention. A monitoring study among participants in the independent and start-up exercise setting was started in 2010. In addition, effectiveness and cost-effectiveness studies have started that follow the participants exercising in the supervised exercise setting, to investigate if this setting is more effective and cost-effective than the start-up exercise setting [159]. In the effectiveness and cost-effectiveness studies, objective as well as self-reported questionnaires are used to measure the participants' physical activity levels. Results of these studies are expected to become available in 2013.

The results of our studies showed that total sitting time, rather than total physical activity time, was associated with BMI. Over the last decade, researchers have become more interested in the role of sedentary behaviour in relation to obesity. Sedentary behaviour can be defined as all activities that do not increase energy expenditure substantially above the resting level, such as sitting, lying down or television viewing [14] and should be measured independently of physical activity and not be defined as merely the absence of physical activity [14, 15]. One of the conclusions of a study by Hamilton and colleagues was that engaging in moderate or vigorous activity a few times a week will not compensate for the potential negative effects of excessive sedentary behaviour [20]. Sedentary behaviour is a risk factor for type 2 diabetes and increased mortality [104], so prevention of this behaviour is important. Further research is needed to explore the role sedentary behaviours plays in relation to obesity and how lifestyle interventions could best incorporate this knowledge to help patients decrease their sedentary time.

Our study measured sedentary behaviour with one question about the amount of time spent sitting per day. Other studies have measured sedentary 
behaviour in a variety of ways. Most studies measured television viewing time (e.g. [116]) while some measured the total sitting time (e.g. [160]), just as we did. Future research requires the development of a sound measurement method for sedentary behaviour, if possible using objective measurements. Several objective methods can be used to measure time spent in sedentary behaviour, for example accelerometers or activity monitors. The advantage of an activity monitor compared to an accelerometer is that it can measure patterns of PA behaviour. An example of a single unit activity monitor is the CAM. A study by Annegarn and colleagues [161] of patients with chronic heart failure or chronic obstructive pulmonary disease showed that wearing a CAM on the upper leg was an accurate method to measure the time spent in weightbearing postures, non-weight bearing postures and dynamic activities in the home environment. The CAM was able to detect time spent in dynamic activity as well as in different postures. Because the CAM is a single-unit activity monitor, it is uncomplicated for patients to use. Patients in the study by Annegarn did not complain about wearing the CAM [161], which means that such single-unit activity monitors are promising tools to objectively measure physical activity and sedentary behaviour, which can be used, for example, to provide patients with behavioural feedback in lifestyle interventions.

In the Netherlands, a lot of many interventions in different fields (including some intended for children, adolescents or adults, and school-based and primary care based programmes) have been developed and tested for their efficacy. These interventions were typically developed and tested using research funding. Some interventions proved to be effective, but after the research funding had stopped, no funding bodies were interested in continuing the programme or implementing it on a large scale. Every year, this results in good and effective interventions not being disseminated. To overcome this barrier, an implementation centre in the Netherlands would be highly beneficial to facilitate such implementation and dissemination trajectories. Such an implementation centre should ideally adopt the role to keep the intervention up to date and to ensure continued dissemination

\section{General Conclusion}

BeweegKuur has provided considerable impetus to the processes involved in the implementation of combined lifestyle interventions in primary care. Various processes (for example the formation of multidisciplinary teams and contacts with local exercise facilitators) were started during the pilot phase of the BeweegKuur programme. It is likely that these processes would also have materialized without BeweegKuur, but that BeweegKuur accelerated this process. The implementation of BeweegKuur demanded a lot of time and energy on the part of the health care providers, partly due to the long pilot period. However, the health care providers involved remained motivated to continue the programme. Furthermore, participants of the intervention were also motivated to increase their physical activity levels, and they appreciated the content of the intervention. The data from the studies among participants indicated that, on 
average, participants did increase their total physical activity time. However, our results also showed that lifestyle counselling should not only aim at an increase in total physical activity time, but also at a decrease in total sitting time. Low-threshold activities such as walking and cycling should be promoted by the LSAs in combined lifestyle interventions. Furthermore, a map showing local exercise facilities in the neighbourhood should be available to the LSAs. Health care providers and other parties have to agree who is responsible for the development and updating of this map, to ensure continued physical activity levels among participants.

In the last decade, the focus of primary health care in the Netherlands has shifted from 'care and disease' towards 'health and behaviour'. In this perspective, the BeweegKuur can be regarded as a good example of this shift. Health care providers in the Netherlands are ready to implement lifestyle interventions such as BeweegKuur. Although they were set to implement and continue the intervention during the pilot period, the question arises if they will go on implementing and continuing lifestyle interventions. 
References 
1. Van der Meer FM, Ligtenberg G, Staal PA (2009) Preventie bij overgewicht en obesitas: de gecombineerde leefstijlinterventie. Diemen: College voor Zorgverzekeringen.

2. Seidell JC (2002) Prevalence and time trends of obesity in Europe. Journal of Endocrinological Investigation, 25(10):816-822.

3. CBS (2009) www.nationaalkompas.nl, [April 2012].

4. Poortvliet MC, Schrijvers CTM, Baan CA (2007) Diabetes in Nederland. Omvang, risicofactoren en gevolgen, nu en in de toekomst. Bilthoven: RIVM.

5. The World Health Organization (2002) The world health report 2002 Reducing Risks, Promoting Healthy Life. Geneva: World Health Organization.

6. Field EA, Coackley EH, Must A, Spadano JL, Laird N, Dietz WH, Rimm E, Colditz GA (2001) Impact of Overweight on the Risk of Developing Common Chronic Diseases During a 10-Year Period. Arch Intern Med, 161:1581-1586.

7. Baan CA, Schoemaker CG, Jacobs-van der Bruggen MAM, Hamberg-van Reenen HH, Verkleij H, Heus S, Melse JM (2009) Diabetes tot 2025. Preventie en zorg in samenhang, Bilthoven: RIVM.

8. Rubin RR, Peyrot M (1999) Quality of life and diabetes. Diabetes Metabolism Research and Reviews, 15:205-218.

9. Marshall SM, Flyvbjerg A (2006) Prevention and early detection of vascular complications of diabetes. BMJ, 333:475-480.

10. Tuomiletho J, Lindström J, Eriksson JG, Valle TT, Hämäläinen H, Ilanne- Parikka P, Keinänen-Kiukaanniemi S, Laakso M, Louheranta A, Rastas M, Salminen V, Uusitupa M (2001) Prevention of type 2 diabetes mellitus by changes in lifestyle among subjects with impaired glucose tolerance. The New England Journal of Medicine, 344(18):1343-1350.

11. Laatikainen $T$, Dunbar JA, Chapman A, Kilkkinen A, Vartiainen E, Heistaro $S$, Philpot B, Absetz P, Bunker S, O'Neil A, Reddy P, Best JD, Janus ED (2007) Prevention of Type 2 Diabetes by lifestyle intervention in an Australian primary health care setting: Greater Green Triangle (GGT) Diabetes Prevention Project. BMC Public Health, 7(249).

12. Mensink M, Feskens EJM, Saris WHM, De Bruin TWA, Blaak EE (2003) Study on Lifestyle intervention and Impaired Glucose Tolerance Maastricht (SLIM): preliminary results after one year. International Journal of Obesity, 27:377384.

13. Fowler MJ. (2007) Diabetes Treatment, Part 1: Diet and Exercise. Clinical Diabetes, 25(3):105-109.

14. Pate RR, O'Neill JR, Lobelo F (2008) The evolving definition of 'sedentary'. Exercise and Sport Sciences Reviews, 36(4):173-178.

15. Rosenberg DE, Bull FC, Marshall AL, Sallis JF, Bauman AE (2008) Assessment of sedentary behavior with the International Physical Activity Questionnaire. Journal of Physical Activity and Health, 5:S30-S44. 
16. Biddle SJH, Gorely T, Marshall SJ, Murdey I, Cameron N (2004) Physical activity and sedentary behaviours in youth: issues and controversies. The Journal of the Royal Society for the Promotion of Health, 124(1):29-33.

17. Sugiyama T, Healy GN, Dunstan DW, Salmon J, Owen N (2008) Joint associations of multiple leisure-time sedentary behaviours and physical activity with obesity in Australian adults. International Journal of Behavioral Nutrition and Physical Activity, 5(35).

18. Healy GN, Matthews CE, Dunstan DW, Winkler EAH, Owen N (2011) (March 1, 2011) Sedentary time and cardio-metabolic biomarkers in US adults: NHANES 2003-06. European Heart Journal, 32(5):590-597.

19. Owen N, Healy GN, Matthews CE, Dunstan DW (2010) Too Much Sitting: The Population Health Science of Sedentary Behavior. Exercise and Sport Sciences Reviews, 38(3):105-113

20. Hamilton MT, Hamilton DG, Zderic TW (2007) Role of low energy expenditure and sitting in obesity, metabolic syndrome, type 2 diabetes, and cardiovascular disease. Diabetes, 56:2655-2667.

21. Knowler WC, Barrett-Connor E, Fowler SE, Hamman RF, Lachin JM, Walker EA, Nathan DM. (2002) Reduction in the incidence of type 2 diabetes with lifestyle interventions or metformin. The New England Journal of Medicine, 346(6):393-403.

22. Mensink M, Blaak EE, Corpeleijn E, Saris WHM, De Bruin TWA, Feskens EJM (2003) Lifestyle intervention according to general recommendations improves glucose tolerance. Obesity Research, 11(12):1588- 1596.

23. Harting J, van Assema P, Ruland E, van Limpt P, Gorgels T, van Ree J, Vermeer F, de Vries NK (2005) Implementation of an Innovative Health Service: A "Real-World" Diffusion Study. American Journal of Preventive Medicine, 29(2):113-119.

24. Uutela A, Absetz P, Nissinen A, Valve R, Talja M, Fogelholm M (2004) Health psychological theory in promoting population health in Päijät-Häme, Finland: First steps toward a type 2 diabetes prevention study. Journal of Health Psychology, 9(1):73-84.

25. Absetz $P$, Oldenburg $P$, Hankonen $N$, Valve $R$, Heinonen $H$, Nissinen $A$, Fogelholm M, Talja M, Uuteka A (2009) Type 2 diabetes prevention in the 'real world': three-year results of the GOAL implementation trial. Diabetes Care, 32(8):1418-1420.

26. Hosper K, Deutekom M, Stronks K (2008) The effectiveness of 'Exercise on Prescription' in stimulating physical activity among women in ethnic minority groups in the Netherlands: protocol for a randomized controlled trial. BMC Public Health, 8(406). 
27. Long BJ, Calfas KJ, Wooten W, Sallis JF, Patrick K, Goldstein M, Marcus BH, Schwenk TL, Chenoweth J, Carter R, Torres T, Palinkas LA, Heath G (1996) A multisite field test of the acceptability of physical activity counseling in primary care: project PACE. American Journal of Preventive Medicine, 12(2):73-81.

28. Visser F, Hiddink G, Koelen M, Van Binsbergen J, Tobi H, Van Woerkum C (2008) Longitudinal changes in GPs' task perceptions, self-efficacy, barriers and practices of nutrition education and treatment of overweight. Family Practice, 25(1):i105-i111.

29. Williams NH, Hendry M, France B, Lewis R, Wilkinson C (2007) Effectiveness of exercise-referral schemes to promote physical activity in adults: a systematic review. British Journal of General Practice, 57(545):979-986.

30. Sorensen J, Sorensen JB, Skovgaard T, Bredahl T, Puggaard L (2010) Exercise on prescription: changes in physical activity and health-related quality of life in five Danish programmes. The European Journal of Public Health, 21(1):5662.

31. Garrett S, Elley CR, Rose SB, O'Dea D, Lawton BA, Dowell AC (2011) Are physical activity interventions in primary care and the community costeffective? A systematic review of the evidence. British Journal of General Practice, 61(584):e125-e133.

32. Sorensen JB, Skovgaard T, Puggaard L (2006) Exercise on prescription in general practice: A systematic review. Scandinavian Journal of Primary Health Care, 24(2):69-74.

33. Swinburn BA, Walter LG, Arroll B, Tilyard MW, Russell DG (1997) Green prescriptions: attitudes and perceptions of general practitioners towards prescribing exercise. British Journal of General Practice, 47(422):567-569.

34. Fortier M, Tulloch H, Hogg W (2006) A good fit: Integrating physical activity counselors into family practice. Canadian Family Physician, 52:942-944.

35. Tulloch H, Fortier M, Hogg W (2006) Physical activity counseling in primary care: Who has and who should be counseling? Patient Education and Counseling 64:6-20.

36. Fokkens AS, Wiegersma PA, Reijneveld SA (2011) Organization of diabetes primary care: a review of interventions that delegate general practitioner tasks to a nurse. Journal of Evaluation in Clinical Practice, 17:199-203.

37. Schmidt M, Absalah S, Stronks K (2006) Wat beweegt de deelnemers? Een evaluatie van het project 'Bewegen Op Recept' in Den Haag. Amsterdam: Academisch Medisch Centrum.

38. Bhattacharyya O, Reeves S, Zwarenstein M (2009) What is Implementation Research? Rationale, Concepts, and Practices. Research on Sociol Work Practice, 19(5):491-502.

39. Rogers E (2003) Diffusion of innovations. Free press: New York.

40. Paulussen TGW (1994) Adoption and implementation of AIDS education in Dutch secondary schools. Maastricht: Maastricht University. 
41. Paulussen T, Wiefferink K, Mesters I (2007) Invoering van effectief gebleken interventies. In: Gezondheidsvoorlichting en gedragsverandering. 5th edition, 151-172.

42. Ajzen I (1991) The theory of planned behaviour. Organizational Behaviour and Human Decision Processes, 50(2):179-211.

43. Bandura A (1977) Self-efficacy: Toward a unifying theory of behavioral change. $P \quad$ sychological Review, 84(2):191-215.

44. Reubsaet A, Reinaerts EBM, Brug J, Van Hooff JP, Van den Borne HW (2004) Process evaluation of a school-based education program about organ donation and registration, and the intention for continuance. Health Education Research, 19(6):720-729.

45. Fleuren MAH, Wiefferink $\mathrm{CH}$, Paulussen TGWM (2002) Belemmerende en bevorderende factoren bij de implementatie van zorgvernieuwingen in organisaties. Leiden: TNO.

46. Grol R, Wensing M (2006) Implementatie, effectieve verbetering van de patiëntenzorg. Maarssenbroek: Elsevier gezondheidszorg.

47. Bowling A. (2002) Research methods in health: investigating health and health services. Second ed., Buckingham Philadelphia: Open University Press.

48. De Weerdt I, Broeders I, Schaars D (2008) Prototype De BeweegKuur. Het beste recept voor uw gezondheid. Een interventie voor de (eerstelijns) zorg om mensen met (een hoog risico op) diabetes mellitus type 2 te begeleiden naar een actievere leefstijl. Bennekom: NISB.

49. Bartholomew KL, Parcel GS, Kok G, Gottlieb NH (2006) Planning Health promotion programs: Intervention Mapping. San Francisco, CA: Jossey Bass.

50. Brug J, Oenema A, Ferreira I (2005) Theory, evidence and intervention mapping to improve behaviour nutrition and physical activity interventions. International Journal of Behavioral Nutrition and Physical Activity, 2(2).

51. Windsor R, Clark N, Boyd R, Goodman R (2004) Introduction to evaluation; measurement in evaluation; process evaluation; formative \& impact evaluation; cost evaluation; planning and evaluation; qualitative evaluation. In: Evaluation of health promotion-health education-disease prevention programs, 3rd edition, New York: McGraw-Hill Publisher; 2004.

52. Sullivan PW, Morrato EH, Ghushchyan V, Wyatt HR, Hill JO (2005) Obesity, Inactivity, and the Prevalence of Diabetes and Diabetes-Related Cardiovascular Comorbidities in the U.S., 2000-2002. Diabetes Care, 28(7):1599-1603.

53. Helmink JHM, Cox VCM, Kremers SPJ (2009) Implementatie van de BeweegKuur: Een pilot studie. Maastricht: Maastricht University.

54. Bemelmans WJE, Wendel-Vos GCW, Bogers RP, Milder I, de Hollander EL, Barte JCM, Tariq L, Jacobs- van der Bruggen M (2008) Kosten-effectiviteit beweeg- en dieetadvisering in de gezondheidszorg. De beweegkuur en dieetadvies onderzocht voor mensen met (hoog risico op) diabetes mellitus type 2. Bilthoven: RIVM. 
55. Smith BJ, Bauman AE, Bull FC, Booth ML, Harris MF (2000) Promoting physical activity in general practice: a controlled trial of written advice and information materials. British Journal of Sports Medicine, 34(4):262-267.

56. Praet SFE, Van Rooij ESJ, Wijtvliet A, Boonman-de Winter LM, Enneking T, Kuipers $\mathrm{H}$, Stehouwer CDA, Van Loon LJC (2008) Brisk walking compared with an individualised medical fitness programme for patients with type 2 diabetes: a randomised controlled trial. Diabetologia, 51(5):736-746.

57. Overgoor L, Aalders M (2004) Big!Move. Evaluatieverslag Big!Move april 2003- juni 2004. Amsterdam: Gezondheidcentrum Venserpolder.

58. Overgoor L, Aalders M, Statius Muller I (2006) Big!Move, beweging in gedrag van patiënt en huisarts. Huisarts \& Wetenschap, 49(1):42-45.

59. GGD Rotterdam-Rijnmond (2008) Zorgmonitor: Rotterdams Leefstijlprogramma Van Klacht naar Kracht. Rotterdam-Rijnmond: GGD.

60. Biddle SJH, Mutrie N (2008) Psychology of physical activity: Determinants, wellbeing and interventions, 2nd edition, London: Routledge.

61. Trost SG, Owen N, Bauman AE, Sallis JF, Brown W (2002) Correlates of adults' participation in physical activity: Review and update. Medicine and Science in Sports and Exercise, 34(12):1996-2001.

62. Harnack L, Block G, Subar A, Lane S, Brand R (1997) Association of cancer prevention-related nutrition knowledge, beliefs and attitudes to cancer prevention dietary behaviour. Journal of the American Dietetic Association, 97(9):957-965.

63. Hollis JF, Carmody TP, Connor SL, Fey SG, Matarazzo JD (1986) The nutrition attitude survey: associations with dietary habits, psychological and physical well-being, and coronary risk factors. Health Psychology, 5(4):359-374.

64. Thomas N, Alder E, Leese GP (2004) Barriers to physical activity in patients with diabetes. Postgraduate Medical Journal, 80:287-291.

65. Deci E, Ryan R (1985) Intrinsic motivation and self-determination in human behaviour. New York: Plenum.

66. Williams GC, McGregor HA, Zeldman A, Freedman ZR, Deci EL (2004) Testing a self-determination theory process model for promoting glycemic control through diabetes self-management. Health Psychology, 23(1):58-66.

67. Silva MN, Markland D, Minderico CS, Vieira PN, Castro MM, Coutinho SR, Santos TC, Matos MG, Sardinha LB, Teixeira PJ (2008) A randomized controlled trial to evaluate self-determination theory for exercise adherence and weight control: rationale and intervention description. BMC Public Health, 8(234).

68. Rothschild ML (1999) Carrots, sticks and promises: a conceptual framework for the management of public health and social issue behaviours. Journal of Marketing, 63:24-37.

69. Williams GC, Minicucci DS, Kouides RW, Levesque CS, Chirkov VI, Ryan RM, Deci EL (2002) Self-determination, smoking, diet and health. Health Education Research, 17(5):512-521. 
70. Miller WR, Rollnick S. (1991) Motivational interviewing: Preparing people to change addictive behaviour. New York: Guilford Press.

71. Festinger L, Schachter S, Back K (1950) Social Pressures in Informal Groups. New York: Harper and Row.

72. Carron AV, Widmeyer WN, Brawley LR (1988) Group cohesion and individual adherence to physical activity. Journal of Sport \& Exercise Psychology, 10(2):127-138.

73. Kwak L, Kremers SPJ, Brug J, Walsh A (2006) How is your walking group running? . Health Education, 106(1):21-31.

74. Spink KS, Carron AV (1992) Group cohesion and adherence in exercise classes. Journal of Sport \& Exercise Psychology, 14(1):78-86.

75. Spink KS, Carron AV (1994) Group cohesion effects in exercise classes. Small Group Research, 25(1):26-42.

76. Praet SFE (2007) Exercise therapy in type 2 diabetes, Dissertation. Maastricht Maastricht University

77. van Osch L, Beenackers M, Reubsaet A, Lechner L, Candel M, de Vries H (2009) Action planning as predictor of health protective and health risk behavior: an investigation of fruit and snack consumption. International Journal of Behavioral Nutrition and Physical Activity, 6(69).

78. Hulscher M, Wensing M, Grol R (2000) Effectieve implementatie: theorieën en strategieën. Den Haag: ZonMw.

79. Wensing M, van Splunteren P, Hulscher M, Grol R (2000) Praktisch nieuw: implementatie van vernieuwingen in de gezondheidszorg. Assen: Van Gorcum.

80. Moore G (2002) Crossing the Chasm. New York: Harper Collins.

81. Helmink JHM, Meis JJM, de Weerdt I, Visser FN, de Vries NK, Kremers SPJ (2010) Development and implementation of a lifestyle intervention to promote physical activity and healthy diet in the Dutch general practice setting: the BeweegKuur programme. International Journal of Behavioral Nutrition and Physical Activity, 7(49).

82. Van Sluijs EMF, van Poppel MNM, Stalman WB, van Mechelen W (2004) Feasibility and acceptability of a physical activity promotion programme in general practice. Family Practice, 21(4):429-436.

83. Fortier MS, Hogg W, O'Sullivan TL, Blanchard C, Reid RD, Sigal RJ, Boulay P, Sweet S, Bisson E, Beaulac J (2007) The physical activity counselling (PAC) randomized controlled trial: rationale, methods and intervention. Applied Physiology Nutrition Metabolism, 32(6):1170-1185.

84. Berwick DM (2003) Disseminating Innovations in Health Care. The Journal of the American Medical Assocation, 289(15):1969-1975.

85. Hoving C, Mudde AN, De Vries H (2007) Intention to implement a smoking cessation intervention into Dutch general practice. Health Education, 107(3):307-315. 
86. Harting J, Rutten GMJ, Rutten STJ, Kremers SPJ (2009) A qualitative application of the diffusion of innovations theory to examine determinants of guideline adherence among physical therapists. Physical Therapy, 89 (3):221-232.

87. Duncan C, Stein MJ, Cummings SR (1991) Staff involvement and special follow-up time increase physicians counseling about smoking cessation: a controlled trial. American Journal of Public Health, 81:899-901.

88. Fleuren M, Wiefferink K, Paulussen T (2004) Determinants of innovations within health care organizations: literature review and Delphi study. International Journal for Quality in Health Care, 16(2):107-123.

89. Mesters I (2009) Motivational Interviewing: hype or hope? Chronic IIIness, 5:3-6.

90. Proper KI, Singh AS, Van Mechelen W, Chinapaw MJM (2011) Sedentary Behaviors and Health Outcomes Among Adults: A Systematic Review of Prospective Studies. American Journal of Preventive Medicine, 40(2):174-182.

91. Levine JA (2007) Nonexercise activity thermogenesis - liberating the lifeforce. Journal of Internal Medicine, 262:273-287.

92. Levine JA, Lanningham-Forster LM, McCrady SK, Krizan AC, Olson LR, Kane PH, Jensen MD, Clark MM (2005) Interindividual Variation in Posture Allocation: Possible Role in Human Obesity. Science, 307:584-586.

93. Santos R, Soares-Miranda L, Vale S, Moreira C, Marques Al, Mota J (2010) Sitting Time and Body Mass Index, in a Portuguese Sample of Men: Results from the Azorean Physical Activity and Health Study (APAHS). International Journal of Environmental Research and Public Health, 7:1500-1507.

94. Fishbein M, Ajzen I (1975) Belief, Attitude, Intention, and Behavior: An Introduction to Theory and Research. Reading, Massachusetts: AddisonWesley Publishing Company.

95. De Vries H, Dijkstra M, Kuhlman $P$ (1988) Self-efficacy: the third factor besides attitude and subjective norm as a predictor of behavioral intentions. Health Education Research, 3(3):273-282.

96. Serrano E, Leiferman J, Dauber S (2007) Self-efficacy and health behaviors toward the prevention of diabetes among high risk individuals living in Appalachia. Journal of Community Health, 32(2):121-133.

97. Salmon J, Owen N, Crawford D, Bauman A, Sallis JF (2003) Physical activity and sedentary behavior: A population-based study of barriers, enjoyment, and preference. Health Psychology, 22(2):178-188.

98. Allen NA. (2004) Social Cognitive Theory in Diabetes Exercise Research: An Integrative Literature Review. The Diabetes Educator, 30(5):805-819.

99. Delahanty LM, Conroy MB, Nathan DM (2006) Psychological Predictors of Physical Activity in the Diabetes Prevention Program. Journal of the American Dietetic Association, 106(5):698-705. 
100. Plotnikoff RC, Lippke S, Courneya KS, Birkett N, Sigal RJ (2008) Physical Activity and Social Cognitive Theory: A Test in a Population Sample of Adults with Type 1 or Type 2 Diabetes. Applied Psychology, 57(4):628-643.

101. Craig CL, Marshall AL, Sjostrom M, Bauman AE, Booth ML, Ainsworth BE, Pratt M, Ekelund U, Yngve A, Sallis JF, Oja P (2003) International Physical Activity Questionnaire: 12-Country Reliability and Validity. Medicine and Science in Sports and Exercise, 35(8):1381-1395.

102. Haskell WL, Lee IM, Pate RR, Powell KE, Blair SN, Franklin BA, Macera CA, Heath GW, Thompson PD, Bauman A (2007) Physical Activity and Public Health. Updated Recommendation for Adults from the American College of Sports Medicine and the American Heart Association. Circulation 116:10811093.

103. Bandura A (1986) Social foundations of thoughts and action: A Social Cognitive Theory. New York: Prentice Hall.

104. Patel AV, Bernstein L, Deka A, Feigelson HS, Campbell PT, Gapstur SM, Colditz GA, Thun MJ (2010) Leisure Time Spent Sitting in Relation to Total Mortality in a Prospective Cohort of US Adults. American Journal of Epidemiology, 172(4):419-429.

105. Hu FB, Leitzmann MF, Stampfer MJ, Colditz GA, Willett WC, Rimm EB (2001) Physical Activity and Television Watching in Relation to Risk for Type 2 Diabetes Mellitus in Men. Archives of Internal Medicine, 161:1542-1548.

106. Dunstan DW, Barr ELM, Healy GN, Salmon J, Shaw JE, Balkau B, Magliano DJ, Cameron AJ, Zimmet PZ, Owen N (2010) Television Viewing Time and Mortality: The Australian Diabetes, Obesity and Lifestyle Study (AusDiab). Circulation, 121:384-391.

107. Krishnan S, Rosenberg L, Palmer JR (2008) Physical Activity and Television Watching in Relation to Risk of Type 2 Diabetes: The Black Women's Health Study. American Journal of Epidemiology, 169:428-434.

108. Paxton RJ, Motl RW, Aylward A, Nigg CR (2010) Physical Activity and Quality of Life-The Complementary Influence of Self-Efficacy for Physical Activity and Mental Health Difficulties. International Journal of Behavioral Medicine, 17(4):255-263.

109. Bandura A (2004) Health Promotion by Social Cognitive Means. Health Education and Behavior, 31(2):143-164.

110. Di Loreto C, Fanelli C, Lucidi P, Murdolo G, De Cicco A, Parlanti N, Santeusanio F, Brunetti P, De Feo P (2003) Validation of a Counseling Strategy to Promote the Adoption and the Maintenance of Physical Activity by Type 2 Diabetic Subjects. Diabetes Care, 26(2):404-408.

111. Wild SH, Roglic C, Green A, Sicree R, King H (2004) Global Prevalence of Diabetes: Estimates for the Year 2000 and Projections for 2030. Diabetes Care, 27(5):1047-1053. 
112. Helmink JHM, Kremers SPJ, Van Brussel-Visser FN, De Vries NK (2011) Sitting Time and Body Mass Index in Diabetics and Pre-Diabetics Willing to Participate in a Lifestyle Intervention. International Journal of Environmental Research and Public Health, 8(9):3747-3758.

113. Kremers SPJ, de Bruijn GJ, Droomers M, van Lenthe F, Brug J (2007) Moderators of Environmental Intervention Effects on Diet and Activity in Youth. American Journal of Preventive Medicine, 32(2):163-172.

114. Rhodes RE, Courneya KS (2003) Modelling the theory of planned behaviour and past behaviour. Psychology, Health \& Medicine, 8(1):57-69.

115. Plotnikoff RC, Lippke S, Courneya K, Birkett N, Sigal R (2008) Physical activity and diabetes: An application of the theory of planned behaviour to explain physical activity for Type 1 and Type 2 diabetes in an adult population sample. Psychology \& Health, 25(1):7-23.

116. Lakerveld J, Dunstan D, Bot S, Salmon J, Dekker J, Nijpels G, Owen N (2011) Abdominal obesity, TV-viewing time and prospective declines in physical activity. Preventive Medicine, 53(4-5):299-302.

117. King AC (2001) Interventions to Promote Physical Activity by Older Adults. The Journals of Gerontology Series A: Biological Sciences and Medical Sciences, 56(suppl 2):36-46.

118. van Stralen MM, De Vries H, Mudde AN, Bolman C, Lechner L (2009) Determinants of initiation and maintenance of physical activity among older adults: a literature review. Health Psychology Review, 3(2):147-207.

119. Resnicow K, Vaughan R (2006) A chaotic view of behavior change: a quantum leap for health promotion. International Journal of Behavioral Nutrition and Physical Activity, 3(1):25.

120. Brug $\mathrm{J}$ (2006) Order is needed to promote linear or quantum changes in nutrition and physical activity behaviors: a reaction to 'A chaotic view of behavior change' by Resnicow and Vaughan. International Journal of Behavioral Nutrition and Physical Activity, 3(1):29.

121. Teixeira PJ, Carraça EV, Markland D, Silva MN, Ryan RM (2012) Exercise, Physical Activity, and Self-Determination Theory: A Systematic Review. International Journal of Behavioral Nutrition and Physical Activity, 9,78.

122. West R, Sohal T (2006) "Catastrophic" pathways to smoking cessation: findings from national survey. BMJ, 332(7539):458-460.

123. Chastin SFM, Granat MH (2010) Methods for objective measure, quantification and analysis of sedentary behaviour and inactivity. Gait and Posture, 31(1):82-86.

124. Chastin SFM, Ferriolli E, Stephens NA, Fearon KCH, Greig C (2011) Relationship between sedentary behaviour, physical activity, muscle quality and body composition in healthy older adults. Age and Ageing, 41(1):111114. 
125. Jansink R, Braspenning J, Van der Weijden T, Elwyn G, Grol R (2010) Primary care nurses struggle with lifestyle counseling in diabetes care: a qualitative analysis. BMC Family Practice, 11(41).

126. Cranney M, Warren E, Barton S, Gardner K, Walley T (2001) Why do GPs not implement evidence-based guidelines? A descriptive study. Family Practice, 18(4):359-363.

127. Grol R, Wensing M (2004) What drives changes? Barriers to and incentives for achieving evidence-based practice. Medical Journal of Australia, 180:S57S60.

128. Ritchie J, Spencer L. (1994) Qualitative data analysis for applied policy research. Bryman A, Burgess R, editors. London: Routledge.

129. Knight KMML, McGowan L, Dickens C, Bundy C (2006) A systematic review of motivational interviewing in physical health care settings. British Journal of Health Psychology, 11:319-332.

130. Rubak S, Dandoek A, Lauritzen T, Christensen B (2005) Motivational Interviewing: a systematic review. British Journal of General Practice, 55:305312.

131. Britt EHS, Hudson SM, Blampied NM (2004) Motivational Interviewing in health care settings: a review. Patient Education and Counseling, 53:147-155.

132. Lawlor DA, Keen S, Neal RD (1999) Increasing population levels of physical activity through primary care: GP's knowledge, attitudes and self-reported practice. Family Practice, 16(3):250-254.

133. McKenna J, Naylor PJ, McDowell N (1998) Barriers to physical activity promotion by general practitioners and practice nurses. British Journal of Sports Medicine, 32:242-247.

134. Helmink JHM, Meis JJM, Kremers SPJ (2010) Een jaar BeweegKuur, en dan? Een onderzoek naar de bevorderende en belemmerende contextuele factoren. Maastricht: Maastricht University.

135. Helmink JHM, Van Boekel LC, Kremers SPJ (2010) Implementatie van de BeweegKuur in de regio. Evaluatie onder ROS-adviseurs. Maastricht: Maastricht University.

136. Helmink JHM, Van Boekel LC, Kremers SPJ (2010) Doorontwikkeling van de BeweegKuur voor Overgewicht \& Obesitas. Maastricht: Maastricht University.

137. Helmink JHM, Van Boekel LC, Kremers SPJ (2011) Implementatie van de BeweegKuur: Monitorstudie onder zorgverleners, najaar 2010. Maastricht: Maastricht University.

138. Helmink JHM, Van Boekel LC, Van der Sluis ME, Kremers SPJ (2011) Lange termijn evaluatie onder deelnemers aan de BeweegKuur: Rapportage van de resultaten van een follow-up meting bij deelnemers. Maastricht: Maastricht University. 
139. Raaijmakers LGM, Helmink JHM, Hamers FJM, Kremers SPJ (2012) Implementatie en continuering van de BeweegKuur: Monitorstudie onder zorgverleners, najaar 2011. Maastricht: Maastricht University.

140. Van Poppel MNM, Chinapaw MJM, Mokking LB, Van Mechelen W, Terwee CB (2010) Physical Activity Questionnaires for Adults: A Systematic Review of Measurement Properties Sports Medicine, 40(7):565-600.

141. National Institutes of Health (2002) NIH guide: Improving diet and physical activity assessment. [April 2012]; Available from: http://grants.nih.gov/grants/guide/pa-files/PAR-03-009.html.

142. Markland D, Ingledew DK (2007) The relationships between body mass and body image and relative autonomy for exercise among adolescent males and females. Psychology of Sport and Exercise, 9:836-853.

143. Fortier MS, Sweet SN, O'Sullivan TL, Williams GC (2007) A self-determination process model of physical activity adoption in the context of a randomized controlled trial. Psychology of Sport and Exercise, 8(741-757).

144. Standage M, Sebire SJ, Loney T (2008) Does Exercise Motivation Predict Engagement in Objectively Assessed Bouts of Moderate-Intensity Exercise?: A Self-Determination Theory Perspective. Journal of Sport \& Exercise Psychology, 30:337-352.

145. Ferrand C, Perrin C, Nasarre S (2008) Motives for regular physical activity in women and men: a qualitative study in French adults with type $\mathbf{2}$ diabetes, belonging to a patients' association. Health and Social Care in the Community, 16(5):511-520.

146. Wilson PM, Mack DE, Grattan KP (2008) Understanding motivation for exercise: A self-determination theory perspective. Canadian Psychology, 49(3):250-256.

147. Teixeira PJ, Silva MN, Coutinho SR, Palmeira AL, Mata J, Vieira PN, Carraça EV, Santos TC, Sardinha LB (2010) Mediators of Weight Loss and Weight Loss Maintenance in Middle-aged Women. Obesity, 18(4):725-735.

148. Silva MN, Vieira PN, Coutinho SR, Minderico CS, Matos MG, Sardinha LB, Teixeira PJ (2010) Using self-determination theory to promote physical activity and weight control: a randomized controlled trial in women. Journal of Behavioral Medicine, 33:110-122.

149. Carraça EV, Silva MN, Markland D, Vieira PN, Minderico CS, Sardinha LB, Teixeira PJ (2011) Body image change and improved eating self-regulation in a weight management intervention in women. International Journal of Behavioral Nutrition and Physical Activity, 8(75).

150. Thelen E, Smith L (1994) A dynamic systems approach to the development of cognition and action. Press MM, editor. Cambridge

151. Moskowitz GB, Skurnik I, Galinski AD (1999) The history of dual-process notions, and the future of preconscious control. In: Chaiken S TY, eds., editor. New York: Guilford Press. 
152. Kremers SPJ, de Bruijn GJ, Visscher TL, van Mechelen W, de Vries NK, Brug J (2006) Environmental influences on energy balance-related behaviors: a dual-process view. International Journal of Behavioral Nutrition and Physical Activity, 3(9).

153. Kremers SPJ (2010) Theory and practice in the study of influences on energy balance-related behaviors. Patient Education Counseling, 79:291-298.

154. Martins RK, McNeil DW (2009) Review of Motivationallnterviewing in promoting health behaviors Clinical Psychology Review, 29(4):283-293.

155. Miller WR, Yahne CE, Moyers TB, Martinez J, Pirritano M (2004) A Randomized Trial of Methods to Help Clinicians Learn Motivational Interviewing. Journal of Consulting and Clinical Psychology, 72(6):1050-1062.

156. Miller WR, Rollnick S (2002) Motivational Interviewing: Preparing people for change. second ed. New York: The Guilford Press.

157. Miller WR, Mount KA (2001) A small study of training in motivational interviewing: does one workshop change clinician and client behavior? Behavioural and Cognitive Psychotherapy, 29:457-471.

158. Linmans, JJ, Spigt, MG, Deneer, L, Lucas, AEM, de Bakker, M, Gidding, LG, Linssen, R \& Knottnerus, JA (2011) Effect of lifestyle intervention for people with diabetes or prediabetes in real-world primary care: propensity score analysis. BMC Family Practice, 12:95

159. Berendsen BAJ, Hendriks MRC, Verhagen EALM, Schaper NC, Kremers SPJ, Savelberg HHCM (2011) Effectiveness and cost-effectiveness of 'BeweegKuur', a combined lifestyle intervention in the Netherlands: Rationale, design and methods of a randomized controlled trial. BMC Public Health, 11:815.

160. Martínez-González MA, Martínez JA, Hu FB, Gibney MJ, Kearney J (1999) Pysical inactivity, sedentary lifestyle and obesity in the European Union. International Journal of Obesity, 23:1192-1201.

161. Annegarn J, Spruit MA, Uszko-Lencer NHMK, Vanbelle S, Savelberg HHCM, Schols AMWJ, Wouters EFM, Meijer K (2011) Objective Physical Activity Assessment in Patients With Chronic Organ Failure: A Validation Study of a New Single- Unit Activity Monitor. Archives of Physical Medicine and Rehabilitation, 92:1852-1857. 
Summary 
The BeweegKuur is a lifestyle intervention tailored to the individual needs of patients, focusing on a change in physical activity and dietary behaviour, to support and prevent the treatment of type 2 diabetes mellitus. The intervention was developed with the aim to become an effective and feasible primary health care based intervention, which in time could be reimbursed under the Dutch basic health insurance scheme. The BeweegKuur originally consisted of a 1-year programme for diabetic and prediabetic patients. Participants are referred by their general practitioner (GP) to a lifestyle advisor (LSA), usually the practice nurse or physiotherapist. Based on specific inclusion criteria and in close collaboration with the patient, an individual exercise programme is designed and supervised by the LSA. This programme can be attended at existing local exercise facilities or (temporarily) under the supervision of a specialized exercise coach or physiotherapist. All participants are also referred to a dietician and receive dietrelated group education. In 2010, the target population of the BeweegKuur changed from prediabetic/diabetic participants into obese/overweight participants. In this thesis, studies related to the development and implementation of the BeweegKuur are described.

Chapter 2 reported on the development process and the content of the BeweegKuur with the use of a model of systematic health promotion planning. The BeweegKuur programme was systematically developed in an evidence- and practicebased process. The factors which explained the motivation among health care providers to implement and continue the BeweegKuur were described in chapter 3. Questionnaire research was used with two measurements among Dutch general practitioners, practice nurses and physiotherapists participating in a pilot study. At baseline, professionals were generally preparing to start with the implementation of the BeweegKuur. At second measurement, all practices were implementing the BeweegKuur. The professionals in our study could be characterized as innovators or early adopters, and inclusion of BeweegKuur in the basic health insurance package was envisaged to persuade other adoption categories to implement the intervention. An intensified focus on skills building (e.g. motivational interviewing skills, general lifestyle counselling skills) was expected to contribute to sustained high-quality implementation of the intervention.

Chapter 4 reported on the relationship between sitting time and Body Mass Index (BMI) in diabetes and pre-diabetes patients willing to participate in the BeweegKuur. Also, demographic and psychosocial associates of the motivation to become more active were identified in this chapter. The measurement instrument was a self-report questionnaire. Results showed that total sitting time was more closely related to $\mathrm{BMI}$ than total physical activity time. Subjects with a higher weight status were more sedentary, but they were also more motivated to be physically active. On the other hand, their self-efficacy to be physically active was lower compared to subjects with a lower weight status. It was concluded that lifestyle interventions to decrease the risk of obesity and type 2 diabetes should not only aim at increasing total physical activity time, but also at reducing the total sitting time. Despite generally high levels of motivation among obese participants, intervention designers and 
intermediaries should be aware of their low level of self-efficacy towards being physically active.

The aim of the study described in chapter 5 was to examine the sociocognitive profile, physical activity level, sitting time, weight status and demographics as baseline predictors of the maintenance of intervention-induced changes in physical activity and sitting time among diabetic and pre-diabetic patients participating in the BeweegKuur with two self-report questionnaires (at baseline and two years after the start of the intervention). Two years after the start of the intervention, respondents, on average, spent more time being physically active. The total sitting time remained almost unchanged between the two measurements. Further exploration showed that respondents who had relatively high levels of physical activity at the start of the intervention, increased their total sitting time, while respondents with relatively low levels of physical activity at the start decreased their sitting time. The socio-cognitive profile did not predict the behaviour of the respondents in this sample of diabetic and pre-diabetic patients. The intervention appeared to be suitable for low-educated people.

Chapter 6 of this thesis reported on a study which determined perceived promoting and impeding factors in the implementation of the BeweegKuur programme for overweight and obese people. The study consisted of focus group meetings with intervention participants, interviews with health care providers and a focus group with dieticians. For some intervention participants, the invitation to participate in BeweegKuur came unexpected, as they had not been diagnosed with a disease. Health care providers were aware of this and took time to explain to participants that the programme was appropriate and safe for them. Participants as well as professionals were generally positive about the feasibility of the programme for overweight and obese people and indicated that the intervention was in accordance with their needs. Both health care providers and patients were motivated to participate in the programme. The multidisciplinary approach and the combination of physical activity and dietary behaviour change can make the BeweegKuur programme a success if potential impeding factors like those identified in the study reported in chapter 6 were overcome.

Chapter 7 gave a reflection on the development, implementation and Dissemination processes of the BeweegKuur in primary care. Some remarkable points were seen in the development, implementation and dissemination processes of the BeweegKuur. First, the implementation of the programme took a lot of time and energy on the part of the health care providers. Second, in the primary health care centres that implemented BeweegKuur, multidisciplinary networks were formed and the contacts with local exercise facilities improved during the implementation. Although it is plausible that without BeweegKuur, some of these processes would also have come about, they would probably have taken much more time and effort and would have remained more local. The BeweegKuur programme facilitated the formation of local networks and of a structure in which combined lifestyle interventions could be implemented at different locations in the Netherlands. The 
findings of the BeweegKuur implementation studies showed that it is important to monitoring the level and form of implementation and sustainability of combined lifestyle interventions in primary care.

The general discussion (chapter 8 ) gives a summary of the main findings and discusses some methodological considerations. In this chapter, implications for practice regarding the content of the BeweegKuur, the implementation of a combined lifestyle intervention and the contextual factors of the implementation of a lifestyle intervention were discussed. Implication for further research are also presented. We conclude that the BeweegKuur has provided momentum to the processes involved in the implementation of combined lifestyle interventions in the Netherlands and that different process regarding the implementation of a lifestyle intervention started during the pilot period. Health care providers in the Netherlands are ready to implement lifestyle interventions such as BeweegKuur. Although they were set to implement and continue the intervention during the pilot period, the question arises if they will go on implementing and continuing lifestyle interventions. 


\section{Samenvatting}


De BeweegKuur is een 'tailored' gecombineerde leefstijlinterventie welke focust op het veranderen van fysieke activiteit en eetgedrag vanuit de eerstelijns gezondheidszorg. Het doel van de BeweegKuur is om personen met (een hoog risico op) diabetes vanuit de eerste lijn te begeleiden naar een actievere leefstijl. De interventie is ontwikkeld met als doel om een uitvoerbare en effectieve interventie te worden en om op den duur opgenomen te worden in de Nederlandse basisverzekering. In 2008 is door NISB stapsgewijs de BeweegKuur ontwikkeld op basis van literatuur, ervaring met reeds bestaande projecten en gesprekken met expertpanels en de doelgroep. In de periode 2008-2009 is de nadruk in de BeweegKuur gelegd op de preventie en behandeling van diabetes mellitus type 2. Vanaf 2009 is de BeweegKuur doorontwikkeld voor patiënten met overgewicht en obesitas.

Deelnemers worden door de huisarts doorverwezen naar een leefstijladviseur (LSA), meestal een praktijkondersteuner of fysiotherapeut. De leefstijladviseur coacht, begeleidt en motiveert de deelnemer de rest van het traject en bepaalt welk beweegprogramma het beste bij de deelnemer past. Het beweegprogramma kan gevolgd worden bij lokale beweegaanbieders of (tijdelijk) onder de supervisie van een beweegcoach of fysiotherapeut. Alle deelnemers worden doorverwezen naar een diëtist en ontvangen voedingsgerelateerde groepseducatie. In dit proefschrift zijn studies gerelateerd aan de ontwikkeling en implementatie van de BeweegKuur beschreven.

Hoofdstuk 2 rapporteert over het ontwikkelproces en de inhoud van de BeweegKuur met behulp van een model voor planmatige gezondheidsbevordering. De factoren die de motivatie van zorgverleners om de BeweegKuur te implementeren en continueren bepalen zijn beschreven in hoofdstuk 3. Met behulp van twee vragenlijsten onder Nederlandse zorgverleners (huisartsen, praktijkondersteuners en fysiotherapeuten) die deelnamen aan de pilotstudie van de BeweegKuur zijn deze factoren gemeten. Tijdens de eerste vragenlijst waren de zorgverleners over het algemeen gereed om met de implementatie van de BeweegKuur te beginnen. Tijdens de tweede meting werd de BeweegKuur door alle deelnemende zorgverleners geïmplementeerd. De zorgverleners in deze studie kunnen gezien worden als 'innovators' of 'early adopters' en de inclusie van de BeweegKuur in de Nederlandse basisverzekering kan ervoor zorgen dat de andere adopter-categorieën overtuigd worden om de BeweegKuur te implementeren. Verder wordt er verwacht dat een intensieve focus op het verwerven van vaardigheden (bijvoorbeeld motivational interviewing vaardigheden en algemene leefstijl counseling vaardigheden) nodig is om een hoge kwaliteit van de invoering van de BeweegKuur te waarborgen.

Hoofdstuk 4 rapporteert over de relatie tussen zitgedrag en de Body Mass Index (BMI) in (pre)diabetespatiënten die wilden deelnemen aan de BeweegKuur. Ook zijn demografische en psychosociale voorspellers van de motivatie om actiever te worden geïdentificeerd in dit hoofdstuk. De resultaten zijn verkregen met een zelfgerapporteerde vragenlijst. Uit de resultaten blijkt dat zitgedrag meer gerelateerd is aan BMI dan de totale beweegtijd. Deelnemers met een hoger gewicht zijn meer sedentair maar ook gemotiveerder om te bewegen. Maar aan de andere kant is de 
eigen effectiviteit om meer te bewegen van deze personen lager in vergelijking met deelnemers met een lager gewicht. De conclusie van deze studie is dat leefstijlinterventies gericht om het risico op overgewicht en diabetes type 2 te verlagen zich niet alleen moeten richten op een verhoging van het beweeggedrag maar ook op het verlagen van het zitgedrag. Ondanks de hoge motivatie van de obese deelnemers in deze studie moeten interventieontwikkelaars zich bewust zijn van de lage eigen effectiviteit ten opzichte van bewegen van deze deelnemers.

Het doel van de studie in hoofdstuk $\mathbf{5}$ is het testen van het socio-cognitieve profiel, beweeggedrag, zitgedrag, gewichtstatus en demografische variabelen als baseline voorspellers van het volhouden van de door de interventie veroorzaakte veranderingen in beweeg- en zitgedrag van (pre)diabeten die deelnamen aan de BeweegKuur. Dit is gemeten met twee zelfgerapporteerde vragenlijsten (bij het begin en twee jaar na de start van de interventie). Twee jaar na de start van de interventie gaven de deelnemers aan meer te bewegen dan bij de start. Het zitgedrag is bijna gelijk tussen de twee metingen. De resultaten laten zien dat bij deelnemers die aan het begin van de interventie relatief veel bewogen, het zitgedrag na 2 jaar hoger is dan aan het begin terwijl deelnemers die aan het begin weinig bewogen juist het zitgedrag verlaagd hebben. Het socio-cognitieve profiel voorspelde het gedrag van de respondenten in deze groep (pre)diabeten niet. Wel blijkt de interventie bruikbaar voor laag opgeleide personen.

Hoofdstuk 6 van dit proefschrift beschrijft een studie welke de ervaren vooren nadelen van de interventie weergeeft voor de implementatie van de doorontwikkelde BeweegKuur voor deelnemers met overgewicht en obesitas. De studie bestaat uit focusgroepen met interventiedeelnemers, interviews met zorgverleners en een focusgroep met diëtisten. Voor sommige deelnemers kwam de uitnodiging om deel te nemen aan de BeweegKuur als een verrassing omdat zij niet gediagnosticeerd zijn met een ziekte. Zorgverleners waren zich hier van bewust en namen de tijd om deelnemers uit te leggen dat het programma geschikt en veilig is. Zowel deelnemers als zorgverleners zijn over het algemeen positief over de bruikbaarheid van de BeweegKuur voor overgewicht- en obesitasdeelnemers en gaven aan dat de interventie overeenkomt met wat zij nodig hebben. Zowel zorgverleners als patiënten waren gemotiveerd om deel te nemen aan het programma. Het multidisciplinaire karakter en de combinatie tussen beweeg- en eetgedrag kan de BeweegKuur tot een succes maken wanneer potentiële nadelen zoals weergeven in deze studie overwonnen worden.

Hoofdstuk 7 geeft een reflectie van het ontwikkelings-, implementatie- en verspreidingsproces van de BeweegKuur in de eerstelijns gezondheidszorg. Er zijn enkele opvallende punten naar voren gekomen tijdens deze processen. Als eerste kostte het implementeren van het programma veel tijd, geld en energie van de zorgverlener. Verder ontstonden er in de praktijken die de BeweegKuur uitvoerden meer multidisciplinaire netwerken in de periode 2007-2011. Ook de contacten met lokale beweegaanbieders verbeterde. Het is mogelijk dat deze processen ook zouden zijn ontstaan zonder de BeweegKuur, maar waarschijnlijk langzamer, moeilijk en 
lokaler. De BeweegKuur heeft er ook voor gezorgd dat het vormen van lokale netwerken op verschillende locaties in Nederland van de grond kwam als mede de totstandkoming van een structuur waarin een gecombineerde leefstijlinterventie geïmplementeerd kan worden. De ervaringen met de BeweegKuur laten zien dat het belangrijk is om inzicht te houden in de mate en vorm van de implementatie en voorzetting van gecombineerde leefstijlinterventies in de eerstelijnszorg.

De discussie (hoofdstuk 8) van dit proefschrift geeft een samenvatting van de belangrijkste bevindingen en enkele methodologische overwegingen bediscussieerd. In dit hoofdstuk komen ook implicaties voor de praktijk met betrekking tot de inhoud van de BeweegKuur, de implementatie van gecombineerde leefstijlinterventies en contextuele factoren met betrekking tot de implementatie van leefstijlinterventies aan bod. Implicaties voor vervolgonderzoek zijn eveneens gepresenteerd. De conclusie van dit proefschrift is dat de BeweegKuur heeft gefungeerd als een vliegwiel om processen ten aanzien van de verspreiding van lokale gecombineerde leefstijlinterventies tot stand te brengen en deze interventies verder door te ontwikkelen. 
Dankwoord 
Het is af! Eindelijk! Klaar! De afgelopen jaren heb ik veel tijd gestopt in het schrijven van dit proefschrift, maar dat was me niet gelukt zonder de hulp van een aantal personen die ik in dit dankwoord wil noemen.

Laat ik beginnen met mijn promotieteam. Nanne, bedankt voor het grondige commentaar op mijn artikelen. Door jou kritische blik werd ik gedwongen om alles nog eens goed te bekijken. Stef, ik denk dat ik geen betere begeleider had kunnen wensen tijdens mijn promotie. Jij gaf dat fietsende meisje een kans om te zien hoe het er in de onderzoekswereld aan toe ging. Een jaar werd twee jaar en uiteindelijk was er genoeg data om een proefschrift over te schrijven. Het geeft een goed gevoel om tijdens het gehele traject zo gesteund te worden. Zeker aan het einde maakte je tijd om commentaar te geven en zag ik op tijden dat ik het niet meer verwachtte toch nog mail met commentaar binnenkomen. Bedankt voor de prettige samenwerking en alle leuke momenten. Hoewel sommige momenten voor mij misschien leuker waren dan voor jou (zoals een confettibombardement (:))! Ik kan nooit meer een kopje chocomel zien zonder aan jou te denken. Kom maar op met je metafoor, ik ben er klaar voor!

Ook wil ik de leescommissie bedanken. Zonder jullie goedkeuring had ik dit proefschrift niet kunnen verdedigen. Bedankt daarvoor en voor het feit dat jullie plaats willen nemen in mijn corona.

Maar ook zonder het NISB zou dit proefschrift er niet geweest zijn. Heel erg fijn waren de korte lijntjes tussen UM en NISB. Als ons tijdens de dataverzameling iets opviel, pakten jullie dit meteen op. Laura, Sofie, Toine, Inge, Liesbeth, Femke, Marloes, Patrick en alle andere NISB'ers die aan de BeweegKuur meewerkten, bedankt voor de prettige samenwerking tijdens dit project. Femke, jou wil ik speciaal bedanken voor alle overleggen die we over de BeweegKuur hebben gehad. Van de hak op de tak, kwamen we overal aan toe. Ook bedankt voor het meeschrijven met mijn artikelen. Hopelijk komen we elkaar in de toekomst nog tegen.

Alle ROS adviseurs, huisartsen, praktijkondersteuners, fysiotherapeuten en diëtisten verdienen een plek in dit dankwoord. Bedankt voor alle tijd die jullie vrijgemaakt hebben voor het beantwoorden van vragen, interviews, vragenlijsten, registratieformulieren en noem maar op. Nog vaak denk ik terug aan de treinritjes die ik door het hele land gemaakt heb. En hoeveel energie ik kreeg van jullie enthousiasme.

Zonder deelnemers geen data, dus voor alle deelnemers: bedankt dat jullie mee wilden doen aan mijn onderzoek en de vragenlijsten invulden. Het enthousiasme van de deelnemers tijdens voedingsbijeenkomsten, beweeggroepen en natuurlijk tijdens de focusgroepen maakten het de reisjes dubbel en dwars waard. En een speciaal woord van dank aan deelnemer 353. Jij hebt onze dag gemaakt! Ben blij dat je deze sport niet in een groep uitvoerde. 
Collega's van GB: bedankt voor alle leuke momenten die ik op de vakgroep gehad heb! Dit maakte het (bijna) iedere dag weer leuk om veel te vroeg in de trein te stappen. En daardoor vlogen de jaren in Maastricht om.

Nog een paar collega's wil ik in het bijzonder bedanken. Lieke, bedankt voor het overnemen van mijn BeweegKuur taken en de fijne samenwerking (en alle informele gesprekjes toen we beide alleen op de kamer zaten). Jessica, bedankt voor het meeschrijven aan mijn artikel. Ondanks dat de analyses wat moeizaam verliepen (ik houd het op mijn zwangerschapsdementie (-)!) is het mede dankzij jouw inzichten een mooi artikel geworden! En natuurlijk Marja, Helga en Leon, bedankt voor alle praktische ondersteuning.

Ook alle assistenten die aan de BeweegKuur gewerkt hebben wil ik bedanken. Vincent, Jessie, Leonieke en Femke, bedankt voor alle hulp bij het uitvoeren van de verschillende onderzoeken. Zonder jullie hulp had ik het niet gered.

En hoe leuk is het om een BeweegKuur team te hebben in Maastricht. Geert, fijn om met jou over de BeweegKuur en onderzoek te sparren of gewoon even binnen te lopen om bij te kletsen. Maar ook het BW team moet hier natuurlijk genoemd worden. Hans, Marike en Brenda die hopelijk gaan bewijzen dat de BeweegKuur kosteneffectief is. Ook jullie bedankt voor de uurtjes overleg over de BeweegKuur.

En dan komen we aan bij de Goulash! Francine, Eline, Eva, Karen en Leonieke. Wat fijn dat ik zo'n leuk groepje mensen om me heen had gedurende de jaren in Maastricht. Hopelijk hebben we nog heel veel etentjes in de toekomst. Francine en Eline, bedankt voor alle kopjes thee, gezelligheid, en natuurlijk het weekendje Londen. Eva, mijn kamergenoot tijdens de laatste maanden. Helaas was je de meeste tijd in het buitenland, maar we hebben er een gezellig kamertje van gemaakt. Wat een leuke dingen hebben wij meegemaakt, met ons reisje naar Vietnam als hoogtepunt! En dan kom ik als laatste bij mijn twee paranimfen. Karen, wat was het fijn om gedurende de afgelopen jaren jouw kamergenoot te zijn. Er zijn maar weinig kamergenootjes die het waarderen als je telefoon of muis vastgeplakt zit $:$;! Bedankt dat je mijn paranimf wil zijn! Leonieke, mijn andere paranimf. Heel veel werk heb je als assistent op mijn project verzet: het verzamelen van data, het schrijven van rapporten en het meeschrijven aan mijn artikelen. Ik ben blij dat ik een andere extreme eter heb gevonden (:)! De treinreisjes door het hele land (en dan vooral het om 22.00 uur 's avonds wachten op Utrecht Centraal als we de aansluiting weer gemist hadden) waren een stuk leuker zo! Ik had me geen betere kamergenootjes kunnen wensen als jullie. En speciaal voor jou Leonieke: ferdeferderff.

Ik wil ook mijn collega's bij de GGD bedanken. Wat leuk dat ik nu bij jullie aan het werk ben en al het enthousiasme zie waarmee jullie werken. Na enkele maanden voel ik me 
al een echte epi-lady! Ike, bedankt dat je mij de kans gaf om bij jullie aan de slag te gaan en dit aan het begin te combineren met het afschrijven van mijn proefschrift.

Floor en Han, wat een geluk heb ik met jullie als ouders! Bedankt voor alle steun tijdens het project. En vooral aan het einde, toen het proefschrift af moest en er oppas nodig was. Mark, Ingrid, Karin en Johan. Ik had geen betere broer, zussen en zwager kunnen wensen. Zoveel gezelligheid om altijd op terug te vallen.

Twan, jij verdient heel veel dank. Samen zijn we hier aan begonnen. Het is niet altijd makkelijk geweest om werken en fietsen te combineren. Gelukkig stond jij onvoorwaardelijk achter me. Ook tijdens de laatste loodjes, waarin ik te veel uren op de werkkamer heb gezeten. En als laatste Feline: jij maakt mijn leven tot een feestje. Door jou was het makkelijker relativeren en tijd voor iets anders te nemen dan werken! 


\section{Curriculum Vitae}


Judith Helmink was born on April 21th, 1983 in Maurik (the Netherlands). After she graduated from high school in 2002 (Revius Lyceum, Doorn), she studied health science at Maastricht University. After she obtained her bachelor degree in 2006, she started a master's programme in Health Education and Promotion at Maastricht University. While studying for her master's degree, which she obtained in 2007, she worked as a student assistant at the department of movement science of this same university. In November 2007, she started her PhD-project at the department of Health Promotion of Maastricht University, which focused on the development and implementation process of the BeweegKuur. Judith Helmink currently works as a researcher at the Regional Public Health Service in Breda. 
Publication List 


\section{Publications from this thesis}

Helmink, J.H.M., Meis, J.J.M., De Weerdt, I., Visser, F.N., De Vries, N.K. \& Kremers, S.P.J. (2010) Development and implementation of a lifestyle intervention to promote physical activity and healthy diet in the Dutch general practice setting: the BeweegKuur programme. International Journal of Behavioral Nutrition and Physical Activity, 7, 49.

Helmink, J.H.M., Kremers, S.P.J., van Boekel, L.C., van Brussel-Visser, F.N. \& de Vries, N.K. (2011) Factors determining the motivation of primary health care professionals to implement and continue the 'Beweegkuur' lifestyle intervention program, Journal of Evaluation in Clinical Practice, 18 (3), 682-688

Helmink, J.H.M., Kremers, S.P.J., van Boekel, L.C., van Brussel-Visser, F.N., Preller, L. \& de Vries, N.K. (2011) The BeweegKuur: A qualitative study towards promoting and impeding factors for successful implementation of a primary health care lifestyle intervention, Family Practice, 29, i68-i74

Helmink, J.H.M., Kremers, S.P.J., van Brussel-Visser, F.N. \& de Vries, N.K. (2011) Sitting Time and Body Mass Index in Diabetics and Pre-Diabetics Willing to Participate in a Lifestyle Intervention, International Journal of Environmental Research and Public Health, 8, 3747-3758

Helmink, J.H.M., Gubbels, J.S., Visser, F.N., De Vries, N.K. \& Kremers, S.P.J.Baseline predictors of maintenance of intervention-induced changes in physical activity and sitting time among diabetic and pre-diabetic patients: A descriptive case series, submitted.

Helmink, J.H.M., Raaijmakers, L.G.M., Rutten, G.M., De Vries, N.K. \& Kremers, S.P.J. Gecombineerde leefstijl interventies in Nederland: ervaringen uit de BeweegKuur, submitted.

\section{Additional publication}

Kremers, S., Helmink, J. (2009). Onderzoek naar de verspreiding en implementatie van de BeweegKuur. Tijdschrift voor Gezondheidswetenschappen, 87, 193-194.

\section{Reports}

Helmink, J.H.M., Cox, V.C.M. \& Kremers, S.P.J. (2009). Implementatie van de BeweegKuur: Een pilot studie. Maastricht, Maastricht University.

Helmink, J.H.M., Meis, J.J.M., Kremers, S.P.J. (2010). Een jaar BeweegKuur, en dan? Een onderzoek naar de bevorderende en belemmerende contextuele factoren. Maastricht, Maastricht University. 
Helmink, J.H.M., van Boekel, L.C. \& Kremers, S.P.J. (2010). Implementatie van de BeweegKuur in de regio. Evaluatie onder ROS-adviseurs. Maastricht, Maastricht University.

Helmink, J.H.M., van Boekel, L.C. \& Kremers, S.P.J. (2010). Doorontwikkeling van de BeweegKuur voor Overgewicht \& Obesitas. Maastricht, Maastricht University.

Helmink, J.H.M., van Boekel, L.C. \& Kremers, S.P.J. (2010). Pilot BeweegKuur overgewicht \& obesitas. Resultaten van een follow-up meting onder deelnemers. Maastricht, Maastricht University.

Helmink, J.H.M., van Boekel, L.C. \& Kremers, S.P.J. (2011). Implementatie van de BeweegKuur: Monitorstudie onder zorgverleners, najaar 2010. Maastricht, Maastricht University.

Helmink, J.H.M., Van Boekel, L.C., van der Sluis, M.E. \& Kremers, S.P.J. (2011). Lange termijn evaluatie onder deelnemers aan de BeweegKuur: Rapportage van de resultaten van een follow-up meting bij deelnemers. Maastricht, Maastricht University.

Raaijmakers, L.G.M., Helmink, J.H.M., Hamers, F.J.M. \& Kremers, S.P.J. (2012). Implementatie en continuering van de BeweegKuur: Monitorstudie onder zorgverleners, najaar 2011. Maastricht, Maastricht University.

Raaijmakers, L.G.M., Helmink, J.H.M., Hamers, F.J.M. \& Kremers, S.P.J. (2012). Inhoudelijke monitor lokale netwerken. Maastricht, Maastricht University. 